IVDSIR/8-1555

\title{
A System for Fire Safety Evaluation of Health Care Facilities
}

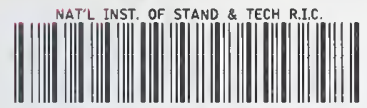

\section{All104 4 7689?}

H. E. Nelson and A. J. Shibe

Center for Fire Research

National Engineering Laboratory

National Bureau of Standards

U.S. Department of Commerce

Washington, D.C. 20234

November 1978

Final Report

Prepared for:

The Department of Health, Education and Welfare

QC

Nashington, D.C. 20201 


\section{A SYSTEM FOR FIRE SAFETY \\ EVALUATION OF HEALTH CARE FACILITIES}

H. E. Nelson and A. J. Shibe

Center for Fire Research

National Engineering Laboratory

National Bureau of Standards

U.S. Department of Commerce

Washington, D.C. 20234

November 1978

Final Report

Prepared for:

The Department of Health, Education and Welfare

Washington, D.C. 20201

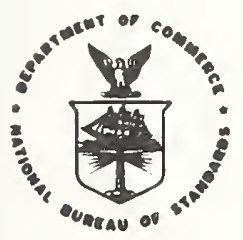

U.S. DEPARTMENT OF COMMERCE, Juanita M. Kreps, Secretary

Dr. Sidney Harman. Under Secretary

Jorden J. Baruch, Assistant Secretary for Science and Technology

NATIONAL BUREAu OF StandaRds, Ernest Ambler, Director 


\section{PREFACE}

This report is an interim product of a joint effort of the Department of Health, Education and Welfare (HEW) and the National Bureau of Standards (NBS), Center for Fire Research. The program is a five-year activity initiated in 1975. It consists of projects in the areas of: decision analysis, fire and smoke detection, smoke movement and control, automatic extinguishment, and behavior in institutional populations in fire situations.

This report, prepared by the Design Concepts Section, developed a methodology for generating an equivalency system for a specific fire safety requirement and a specific example for a system which provides equivalency to the minimum life safety requirements for the health care facilities as prescribed by Life Safety Code 101-1973. 
LIST OF FIGURES $\ldots \ldots \ldots \ldots \ldots \ldots \ldots \ldots \ldots \ldots \ldots \ldots \ldots \ldots \ldots \ldots \ldots$

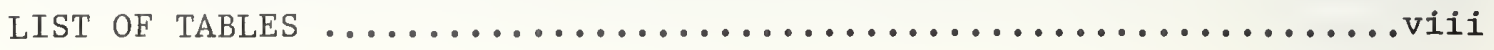

Abstract $\ldots \ldots \ldots \ldots \ldots \ldots \ldots \ldots \ldots \ldots \ldots \ldots \ldots \ldots \ldots \ldots \ldots \ldots \ldots \ldots \ldots$

1. INTRODUCTION $\ldots \ldots \ldots \ldots \ldots \ldots \ldots \ldots \ldots \ldots \ldots \ldots \ldots \ldots \ldots \ldots \ldots \ldots \ldots$

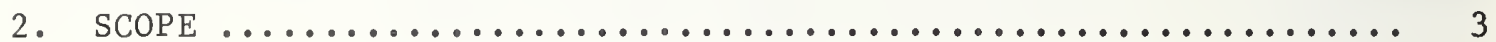

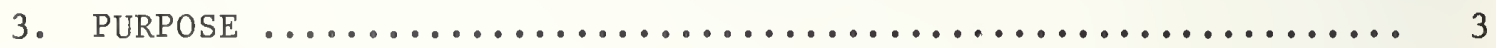

4. EQUIVALENCY CONCEPT ............................ 4

5. PROJECT METHODOLOGY ............................ 5

6. SYSTEMS DESCRIPTION ............................. 6

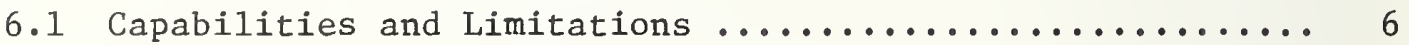

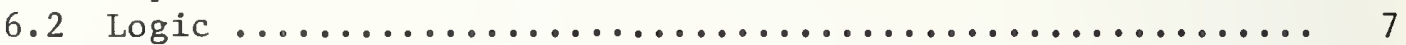

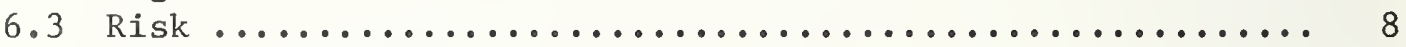

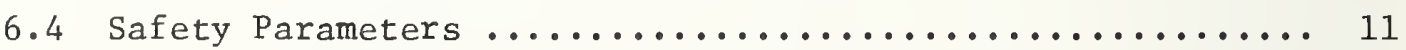

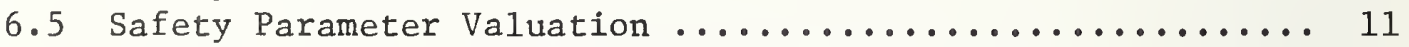

6.6 Relating Safety Parameter Values to Life Safety

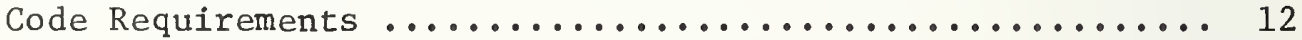

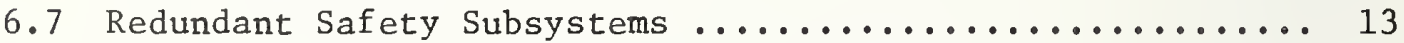

6.8 Overall Safety Evaluation of a Fire/Smoke Zone ......... 14

7. FIRE/SMOKE ZONE EVALUATION WORK SHEET FOR HEALTH CARE

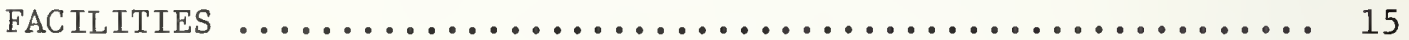

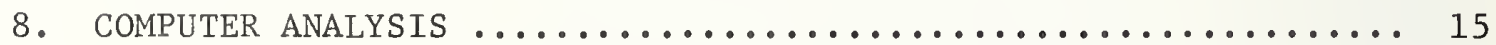

9. EVALUATION SYSTEM ANALYSIS ........................ 15

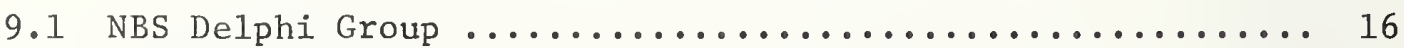

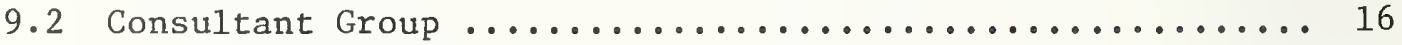

9.3 Inter-Group Relationships .................... 17

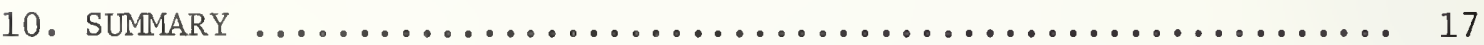

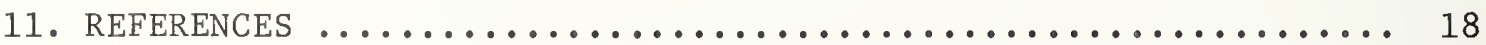




\section{CONTENTS (Cont'd)}

Page

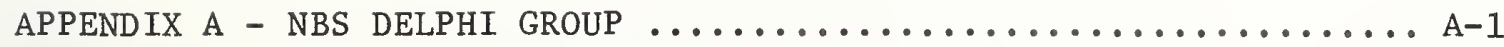

APPENDIX B - CONSULTANT GROUP ............................ B-1

APPENDIX C - CROSS CORRELATION BETWEEN EVALUATION SYSTEM

AND THE LIFE SAFETY CODE ...................... C-1

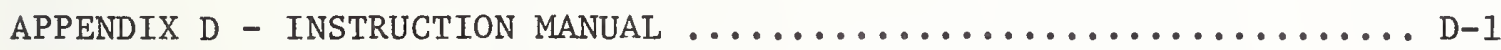

APPENDIX E - SAMPLE COMPLETED FORMS ........................

APPENDIX F - COMPUTER PROGRAM ............................ F-1 
Figure 1. Occupancy risk parameter factors................ 19

Figure 2. Safety parameters.......................... 20

Figure 3. Safety parameters values.................... 21

Figure 4. Life safety code requirements (non-sprinklered)...... 22

Figure 5. Life safety code requirements - new, nonsprinklered, one story facility....................... 23

Figure 6. Life safety code requirements - new, non-spinklered, more than one story facility.................

Figure 7. Life safety code requirements - existing, nonsprinklered, one story facility.............. 25

Figure 8. Life safety code requirements - existing, nonsprinklered, more than one story facility......... 26

Figure 9. Life safety code requirements (sprinklered)........ 27

Figure 10. Life safety code requirements - new, sprinklered, one

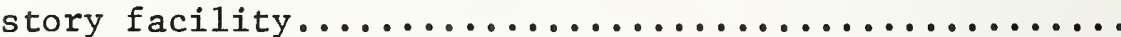

Figure 11. Life safety code requirements - new, sprinklered, more

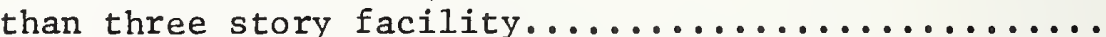

Figure 12. Life safety code requirements - existing, sprinklered, one story facility....................... 30

Figure 13. Life safety code requirements - existing, sprinklered,

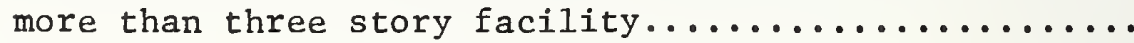

Figure 14. Life safety code requirements - two and three

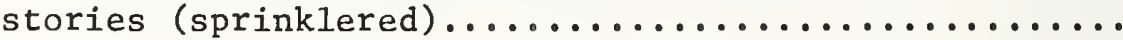

Figure 15. Life safety code requirements - new, sprinklered, two

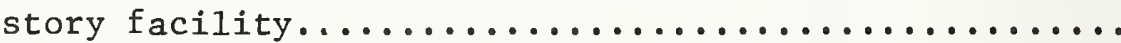

Figure 16. Life safety code requirements - new, sprinklered, three

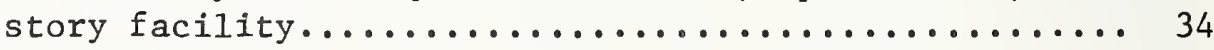

Figure 17. Life safety code requirements - existing, sprinklered,

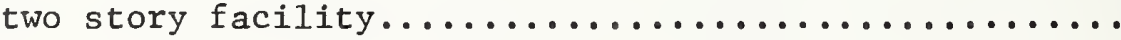

Figure 18. Life safety code requirements - existing, sprinklered, three story facility....................... 


\section{LIST OF FIGURES (Cont'd)}

Page

Figure 19. Individual safety evaluations................. 37

Figure 20. Minimum containment safety (non-sprinklered)....... 38

Figure 21. Minimum extinguishment safety (non-sprinklered)..... 39

Figure 22. Minimum people movement safety (non-sprinklered)..... 40

Figure 23a- Fire/smoke zone evaluation work sheet for health

23d care facilities...................................

Figure $23 e$ Facility fire safety requirements work sheet....... 45

Figure 24. Liaison with review groups................. 46

Figure A-1. Safety parameters - Delphi format.............. A-7

Figure A-2. Safety parameters matrix.................... A-8

Figure A-3. Safety parameters values..................... A-9

Figure A-4. Individual safety evaluations.................. A-10

Figure E-1a-

E-1d Large hospital.......................... E-2-E-5

Figure E-2a

E-2d Small hospital.......................... E-6-E-9

Figure E-3a-

$\mathrm{E}-3 \mathrm{~d}$ Nursing home.................................... 
Table A-1. NBS Delphi group ...........................

Table B-1. Consultant group .............................. B-1

Table B-2. Dates of meetings and attendance .................. B-3

Table C-1. Cross reference to the Life Safety Code (LSC - 1973)... C-2

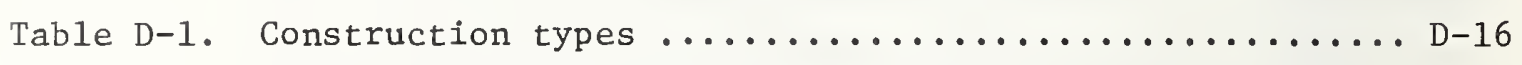

Table D-2. Flame spread classification for wall and ceiling

finishes .............................. D-19

Table D-3. Fire resistive partition details ................ D-20

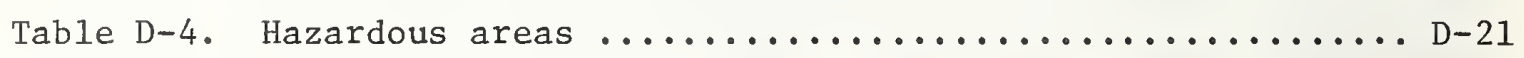

Table F-1. Fire zone evaluation ........................ F-4 


\title{
A SYSTEM FOR FIRE SAFETY \\ EVALUATION OF HEALTH CARE FACILITIES
}

H. E. Nelson and A. J. Shibe

\begin{abstract}
A quantitative evaluation system for grading health care facilities in terms of fire safety is described. The system can be used to determine how combinations of widely accepted fire safety equipment and building construction features may provide a level of safety equivalent to that required by the widely accepted Life Safety Code of the National Fire Protection Association. The system will provide flexibility to both the designer of new facilities and to the renovator of existing health care facilities.

Three major concepts form the basis for code equivalency:

a. Occupancy Risk - the number of people affected by a given fire, the level of fire they are likely to encounter, and their ability to protect themselves.

b. Building Safety Features - the ability of the building and its fire protection systems to provide measures of safety commensurate with the risk.

c. Safety Redundancy - in-depth protection, through the simultaneous use of alternative safety methodologies such as containment, extinguishment, and people movement methodologies. The design of the complete fire safety system is intended to ensure that the failure of a single protection device or method will not result in a major failure of the entire system.

In this system, equivalency is judged to exist when the total impact of the occupancy risk factors and the compensating building safety features produce a level of safety equal to or greater than that achieved by rigid conformance to the explicit requirements of the NFPA Life Safety Code. In this evaluation, safety performance is gauged both in terms of overall safety impact and depth of redundance.
\end{abstract}


Key words: Risk analysis; fire safety; safety equivalency; health care facilities; Life Safety Code; smoke detection; automatic sprinklers; building construction; interior finishes; building codes; hospitals; nursing homes; Delphi Method; safety evaluation.

\section{INTRODUCTION}

The ideal goal of life/fire safety design is to prevent all fire deaths, injuries and losses under all imaginable circumstances. Practically, however, society can neither forestall all loss of life nor spend limitlessly to avert loss of life due to fire. Building codes are designed to provide a minimum acceptable level of life safety at a cost society is able and willing to support. The Life Safety Code of the National Fire Protection Association (NFPA No. 101) is a voluntary code widely accepted for setting acceptable fire safety levels. The code provides fixed solutions for life safety in designated occupancies, but allows "equivalent" solutions. However, it does not define alternative solutions nor provide a mechanism for evaluating equivalence.

The Center for Fire Research with support from the Department of Health, Education and Welfare has developed a system for determining how combinations of widely accepted fire safety systems and arrangements may provide a level of safety equivalent to that required in the 1973 Life Safety Code. The system provides flexibility to the designer of new facilities and to the renovator of existing health care facilities.

The major concepts forming the basis for code-equivalence are:

a. Occupancy Risk - the number of people affected by a given fire, the level of fire they are likely to encounter, and their ability to protect themselves.

b. Building Safety Features - the ability of the building and its fire protection systems to provide measures of safety commensurate with the occupancy risk.

c. Safety Redundancy - in-depth (redundancy) protection, through the simultaneous provision of alternative safety methodologies such as containment, extinguishment, and people movement methodologies. Each methodology provides at least its own independent minimum code level of protection. The design of the complete fire safety system is intended to ensure that the failure of a single protection device or method will not result in a major failure of the entire system.

An adequate life safety system must include a building safety system commensurate with the occupancy risk. The Fire Safety Evaluation System provides a model for evaluating the fire risk in a building, by incorporating factors such as mobility of people, number of people at 
risk, height of building, etc. The calculated risk level provides a minimum target for which levels of protection must be provided by the nature of the building design supplemented by appropriate passive and active fire protection devices.

The evaluation system is also a technique for obtaining a quantitative measure of the level of safety provided by a protected building. This level is determined from an evaluation of the various construction elements and fire protection features. The system measures both the gross level of safety and a set of safety subsystems related to containment, extinguishment and people movement. These subsystems are individually graded to evaluate the depth of redundancy in the building fire safety system. The evaluation system is a mechanism by which the designer can combine a wide variety of fire safety elements into a health care facility plan that provides the level of fire safety required to balance the calculated risk levels.

\section{SCOPE}

This evaluation system applies to institutional buildings used for health care purposes involving sleeping facilities for the occupants. In its present form, it was not designed for outpatient clinics or other facilities where all of the occupants are normally awake. The system as presented in this report was also not designed to evaluate penal institutions and has not been proof tested against that type of occupancy.

The system to be described covers all of the aspects of building fire safety currently covered by the Life Safety Code. A few of the items related to built-in utility systems and to operational features and furnishings (fire evacuation plans, fire drills, draperies, etc.) have been excluded from the grading systems. However, these are covered as mandated items in an attachment to the evaluation form. To be considered as having demonstrated equivalent fire safety, it is necessary that the facility satisfy these requirements in addtion to the basic fire safety evaluation.

\section{PURPOSE}

The purpose of the evaluation system is to provide a technically based mechanism applicable to health care facilities for:

a. Evaluation of an existing health care facility to determine how the actual level of fire safety in that facility compares to the level of fire safety that would be provided by explicit conformance to the requirements of the 1973 Life Safety Code.

b. Evaluation of various alternative approaches available to upgrade an existing facility to a level of fire safety that meets or exceeds the level prescribed by the Life Safety Code. 
c. Evaluation of a proposed new facility design to determine how its level of fire safety would compare to that required for such a facility by the Life Safety Code. In this aspect the system can also be used as a design instrument to determine various alternatives that can be considered.

d. Evaluation of the cost effectiveness of alternate fire safety designs and methodologies.

e. Quantitative evaluation of the relative degree of protection involved in a facility or a design feature, as compared with that required by the Life Safety Code.

\section{EQUIVALENCY CONCEPT}

The evaluation system provides a method for determining the design features needed to provide equivalence to the 1973 Life Safety Code. Equivalency for the purpose of the system is judged by comparing the total fire safety level prescribed by the Life Safety Code for health care facilities to the actual safety level in a particular design or an actual facility.

The Life Safety Code represents a consensus view by knowledgeable professionals of the minimum standard for fire safety necessary to safeguard the public interest.

Equivalency determination is based on the concept that, while the Life Safety Code does not include a specific statement of the level of safety provided, it is possible by examination of the Code requirements to establish a base line for comparing the level of safety provided by strict conformance to its requirements with the level of safety provided by alternative systems of safeguards. This comparison can be made on the basis of the total safety performance of the building, including all of its safeguards, without making direct comparisons between a specific Code requirement for an element and the corresponding element as it exists in the building. The concept is suitable for use with any of the recent editions of the NFPA Life Safety Codes for 1967, 1970, 1973 and 1976. The following reference sections are quoted from NFPA 101-1973:

"1-3118. Nothing in this Code is intended to prevent the use of new methods or new devices, providing sufficient technical data is submitted to the authority having jurisdiction to demonstrate that the new method or device is equivalent in quality, strength, fire resistance, effectiveness, durability, and safety to that prescribed by this Code."

"1-4113(c). The specific requirements of this Code for existing buildings may be modified by the authority having jurisdiction to allow alternative arrangements that will secure as nearly equivalent safety to life from fire as practical, but in no case shall the modification be less 
restrictive or afford less safety to life than compliance with the corresponding provisions contained in this Code for existing buildings. (See also 1-3118)."

Similar paragraphs also exist in the 1967, 1970, and 1976 editions of the Life Safety Code.

Evaluation of equivalency of fire safety is through consideration of three separate concepts. The first is occupancy risk, judged primarily on: how many people are susceptible to a single fire exposure (level of risk), what is their capability to safeguard themselves, and what is the nature of the exposure to which they are being subjected. The second is the capacity of the building and its fire protection systems to provide a safe environment commensurate with the risk. The third is the degree of redundant capabilities to insure the preservation of safety in case of the failure of any one safeguard or method.

\section{PROJECT METHODOLOGY}

Development of the fire safety evaluation system consisted of three operations: system design, professional judgment review and critique, and system testing.

a. System Design. This consisted of: (1) analysis of the stated requirements of the 1973 Life Safety Code versus the fire safety function(s) of each requirement, (2) organization of the results of this analysis into a format suitable for obtaining professional judgments of the comparative worth of the fundamental code requirements relative to the Life Safety Code objectives for health care facilities, (3) development of a computer program to evaluate alternative designs and fire protection systems, and (4) iterative incorporation of system changes resulting from the professional judgment review and system tests.

b. Professional Judgment Review. The professional judgment review was made by two different groups: (1) an NBS group, through the mechanism of a "Delphi" exercise (see appendix A for description of the NBS Delphi operation), and (2) an outside review group (see appendix B). The Delphi group (an ad hoc group of qualified fire protection engineers from the Center for Fire Research, NBS) refined the format, established initial values of the safety parameters, and provided judgment values for selecting individual safety parameters in the redundancy evaluations. The outside consulting group consisted of prominent persons in the field of regulation and specification of fire safety requirements in health care facilities. The group provided broad-based technical and judgmental information for improving both the format and the final values assigned to the safety parameters and the redundancy factors. 
c. System Testing. The testing involved a series of exercises to determine the validity of the fire safety evaluation system. These exercises included: field tests of actual facilities by NBS personnel; examinations of many evaluation work sheets completed by health care facilities owners and engineering staff, and by code certification and inspection authorities; and, computer analysis of alternative design systems.

\section{SYSTEMS DESCRIPTION}

\subsection{Capabilities and Limitations}

The system that has evolved from this effort provides a means of mixing recognized and proven fire safety systems and approaches and evaluating these mixes in terms of the overall fire safety performance of a facility. It permits comparative evaluations of the fire risks and fire safety factors actually present in individual facilities or design. Those features that are in excess of minimum safety requirements are given appropriate credit, reflecting the degree of additional safety actually provided. The credit however is limited in its application to the methodological areas where the safeguards provide credible improvements in safety. Conversely, features that increase one or more aspects of fire risk are appropriately charged for their detrimental impact on safety. The result is intended to be an assessment of total safety performance as compared to a minimum code safety level, which provides opportunity for cost reduction, wider choice of design alternatives, and operational flexibility at greater levels than currently available through explicit compliance with the Life Safety Code.

The most important limitations of the evaluation system are:

a. As presently developed the evaluation system applies only to health care facilities of the types covered under Chapter 10 of the 1973 Life Safety Code.

b. The results are expressed in equivalency to the level of safety achieved by the Life Safety Code, and should not be construed as a measure of total or absolute fire safety.

c. The system, like all existing methods for regulating or evaluating fire safety, is only partially supported by technical information or statistics. The professional judgment of experts in a series of balanced peer-consensus groups is used to bridge the technology gaps.

d. In general the evaluation system is limited to evaluating the interrelationships of those fire safety methodologies and approaches that are defined in the Life Safety Code. There is no basis in the system for accommodating completely innovative approaches (such as automatic venting at the point of fire or 
the use of halogenated gases as a general protection system) for which equivalency with the Life Safety Code cannot be determined.

e. While the parameter measurements of the system cover built-in structural materials and elements, space arrangement, and fire protection systems and devices, the system does not permit alternate approaches to meeting the Life Safety Code requirements for:

(1) Utilities such as heating, air conditioning, electrical, and incinerator systems.

(2) Furnishings such as draperies, curtains, wastebaskets, and beds.

(3) Administrative activities such as emergency plans and fire drills.

When using the evaluation system, the existing requirements in these three areas are applied in the traditional manner of explicit conformance with the established standards and requirements as described and/or referenced in the Life Safety Code. A form to accomplish this in terms of ten specific requirements is shown in figure $23 \mathrm{e}$.

\subsection{Logic}

The logic of the system is that the level of risk imposed upon persons in a facility must be met by a system of safeguards that provide sufficient safety to protect against that risk, using several interacting but separate design approaches.

The evaluation is made on a "FIRE/SMOKE ZONE" basis. This is in recognition of both the history of fires in health care facilities and the traditional arrangements of patient care areas. The evaluation of fire safety is relevant both to the capability of patients surviving fire initiated in such a unit, and to the ability of the unit to exclude the impact of fires external to it. The term "fire/smoke zone" is defined as a space separated from all other spaces by floors, and by horizontal exits or smoke barriers. Where a floor is not subdivided by horizontal exits or smoke barriers, the entire floor is the zone.

The evaluation system applies this logic to each patient use fire/ smoke zone through the following steps.
a. Measure RISK.
b. Measure overa11 (GENERAL) level of safety. 
c. Measure depth (redundancy) of safeguards in terms of:

(1) Fire CONTAINMENT capabilities.

(2) EXTINGUISHMENT, suppression, and control capabilities.

(3) PEOPLE MOVEMENT and other occupant protective features.

d. Determine equivalency to the prescribed requirements of the Life Safety Code. Equivalency occurs when the values as measured by this system are such that:

(1) The GENERAL safety level equals or exceeds the occupancy RISK level, and

(2) The CONTAINMENT, EXTINGUISHMENT and PEOPLE MOVEMENT safety levels each independently equals or exceeds the minimum value corresponding to the level of that category required by the Life Safety Code.

\subsection{Risk}

In establishing a system for evaluating risk, it is recognized that there is a basic level of fire risk inherent in every health care facility. It is also recognized that the amount of furniture, equipment, and supplies (plus the arrangement of these within the space available) depends on the occupant and is not quantified in the safety equivalency measurement. The evaluation system base line for occupancy risk rests on the assumption that the furniture*, equipment, and supplies will be combustible, most adversely located from a fire-safety standpoint, and typical of those normally found in health care facilities.

The factors used to judge the variations in fire risk are given in figure 1. They are applied to individual fire/smoke zones and cover the following risk controlling parameters: the number of patients in the zone, their degree of mobility, their average age, the ratio of patients to attendants, and the height of the zone above street level. These five specific occupancy risk parameters were initially chosen based on the experience and judgment of selected members of the staff of the Fire Safety Engineering Division, Center for Fire Research, and because they are considered to represent the occupancy variables that control the risk in health care facilities. The assessment of the specific parameters and the determination of their relative importance was also based on judgment plus the exercising of the system on test cases to reveal inconsistencies or deviations from accepted safety practice.

The Occupancy Risk Factor for any health care building is the product of five individual risk parameters factors based on the risk factor values shown in figure 1 .

*Facility furniture could be expected to vary ad hoc so they cannot be considered as known in a system analysis. 
The minimum risk conditions have been defined as: a zone containing fewer than five patients, all of whom are of sufficient health to be considered fully mobile and capable of evacuating themselves, their average age being less than 65 and over one; a ratio of patients to attendants of 2:1 or less; and the zone located on the first floor of the building. This condition is assigned an occupancy risk factor of 1.1 .

In contrast, the conditions evaluated as representing the upper range of risk as contemplated by the Life Safety Code are based on: more than 30 patients in the zone, all of whom are unable to move without assistance; their average age is over 65 or under one; a ratio of patients to attendants of 10:1 or more; and the zone located above the sixth floor or in the basement. This was assigned an occupancy risk factor of 18. Greater risk values are assigned to patients who cannot be moved and to fire zones which are unstaffed. The maximum possible risk has a factor of 69 .

The risk factors were chosen and weighed in descending order of maximum impact as follows:

a. Patient Mobility. The single most important factor controlling risk in a health care facility is the degree to which patients must be assisted in taking actions necessary for their safety. The level of capability in health care facilities will vary from patients who, if informed or directed, will be able to take positive self-protecting actions to those patients who cannot be moved or cannot take the simplest actions to safeguard themselves. In the measurement of occupancy risk factors the least mobile category of patient expected in the zone determines the risk factor for that zone. The rationale for this approach is that if a zone accepts any patient with a reduced mobility status, at any time it may increase the number of those patients. For patients who cannot be moved because of extreme danger of death or serious harm, this condition is considered to be a major risk and a very high risk factor (4.5) is imposed. With this high risk factor, the system requires inclusion of fire safeguards which exceed the normal minimum requirements of the Life Safety Code sufficient to compensate for total lack of movability. This is one of two situations where it is possible for a building, which explicitly complies with the Life Safety Code, to fail to meet the minimum requirements as determined by the evaluation system.

b. Patient Density. The risk factor for occupant density (number of patients within the zone) takes into account both the inherent increase in the maximum fire death potential that occurs as the number of patients in a zone increases, and the problems involved in handing larger numbers of patients during an emergency. 
c. Fire/Smoke Zone Location. This risk factor relates to fire department accessibility to the fire. The rating system recognizes the inherent advantage of a first floor zone. It also recognizes the problems of evacuation from higher floors and the virtual impossibility of using external fire fighting efforts above the sixth floor in any building. The risk factor value for zones in basements is the same as for zones at or above the seventh floor.

d. Ratio of Patients to Attendants. This risk factor recognizes the importance to patient safety of attendants immediately available to respond in an emergency. The emergency actions that may be undertaken by the staff include detection, alarm, fire extinguishment, confinement of the fire, establishing barriers between the patients and the fire (e.g. closing patient room doors), rescue, emergency medical aid, and other related functions. A few of these functions, such as detection and alarm, may not be critically related to the ratio of attendants to patients while those functions related to rescue and the closing of patient room doors have a strong relationship to the staffing ratio. The staffing ratio considered is based on the minimum staffing level that would be immediately available (normally night hours). In establishing the risk charges, the charge considered equivalent to the most severe case contemplated under the Life Safety Code was assumed at a patient/ attendant ratio of 11 or more, but where there was at least one attendant constantly in or immediately adjacent to and in full observation of the zone. The Life Safety Code is actually silent on this matter and could even be interpreted to permit a situation where there were no attendants in or adjacent to the zone. Such a condition was considered to be a major fire risk and a high risk factor (4.0) is imposed in a situation where patients are left without immediate nursing staff assistance. With this high risk factor, the system requires inclusion of sufficient fire safeguards to reasonably compensate for the lack of human supervision. This is a second situation in which it is possible for a building with fire protection devices to explicitly comply with the Life Safety Code, but to fail to meet the minimum requirements as determined by the evaluation system.

e. Patient Average Age. This risk factor recognizes the increased susceptibility of the elderly and infants up to one year of age to physical harm by smoke particles, gaseous combustion products and heated air. The rating assigns a larger risk factor (1.2) to fire zones occupied by a population whose mode is above 65 or below one year. Basically, imposition of this charge will provide additional safety protection in nursing homes for the aged and nurseries. 


\subsection{Safety Parameters}

The general safety factors are measures of those building and fire protection features that bear upon the safety of patients (and other occupants) who may be in the particular fire/smoke zone at the time of a fire.

The safety parameters were selected by examining the specific code element requirements for health care facilities, Chapters 10 and 17 of the 1973 Life Safety Code, and by evaluating the impact of various elements of the Code. The selected safety parameters were modified first by the NBS Delphi panel and later by the consultant groups. The selected safety parameters are shown in figure 2. (See appendix $C$ for an accounting of the inclusion of code elements in the evaluation system.)

Each of the safety parameters was analyzed. Where the current Code requirements recognize several different levels of a parameter (e.g. the Life Safety Code recognizes eight different types of construction), the most important alternatives were listed. In addition, conditions likely to be encountered in situations failing to meet the explicit Code requirements, and conditions exceeding those required by the Code but available for increased protection were also listed. Figure 3 shows the final "matrix" form of the breakdown of the 13 selected safety parameters, each having three to seven subdivisions.

The safety parameters are designed to constitute a complete assembly of all of the basic building factors determining the level of safety in a health care facility for which equivalency could be expressed. In addition, we collected and attached to the inspection form an additional series of items required by the Life Safety Code but outside the scope of the equivalency covered by the listed safety parameters. These relate primarily to building utilities, operational features and furnishings and they are listed in part e of figure 23.

\subsection{Safety Parameter Valuation}

In order to provide a method of bringing the best available consensus judgment and experience together to judge the relative impact on general safety of each of the parameters in each of the potential conditions listed, a Delphi type peer group was established. This peer group consisted of members of the Fire Safety Engineering Division, Center for Fire Research, NBS, with the greatest background and experience in the application of fire protection engineering principles and practices to buildings. The membership and basis for the Delphi approach are covered in appendix $A$.

Each member of the group was provided with copies of the initial matrix similar to the one shown in figure 3, but without numerical parameter values. Each person then evaluated the relative importance of each item in the entire matrix of parameters without consultation with other members of the group. The members of the Delphi group were advised that the risk being considered covered new and existing health care 
facilities and that the objective was a system to measure equivalency with the 1973 Life Safety Code. The value judgments made by this group are, therefore, considered to be based on the character and needs of patients in health care facilities and the current approach to these embodied in the Life Safety Code. In addition, each member of the group evaluated separately the same matrix in relation to the redundant subsystems which are discussed in section 7.7. In executing the matrix each peer group member was requested to assign a value of +10 to that safety parameter element (or level) considered to be the single most important to safety to life and to compare all other elements in the matrix to that base. A zero value represented a neutral condition; i.e. a safety parameter at this level would not increase or decrease the safety conditions of a fire zone. Negative values represented deficiencies; i.e. safety parameters at this level decreased the safety conditions of a fire/smoke zone.

After an initial analysis of the results, the peer group was asked to meet in conference on several occasions. The peer group on those occasions deviated from the traditional Delphi approach but instead reviewed differences and concepts, with a view to achieving consensus agreement on categories and on selection of the numerical values.

Several categories were modified and qualified. A significant adjustment was to shift the numbers so that a base line would be established in which negative charges would not be made against any general safety parameter that was in explicit conformity with the requirements of the 1973 Life Safety Code.

\subsection{Relating Safety Parameter Values to Life Safety Code Requirements}

The relationship between the safety parameter values and the code requirements was established by summing the value of all of the credits and deficits of the safety parameter elements for a health care facility that exactly met all of the requirements prescribed by the 1973 Life Safety Code. Attempts to do this disclosed that the Life Safety Code actually had eleven sets of requirements, seven for sprinklered facilities and four for non-sprinklered facilities (see figures 4 through 18). Based on the relative value of protection methodologies developed by the Delphi group and refined by the review processes described later in this report, the levels of safety prescribed by these requirements are: 


\section{General Safety Value Required \\ Non-Sprinklered \\ Buildings \\ Sprinklered \\ Buildings}

1. New buildings 1-story in height.

13

18

5

9
16

$23(a)$

8

$16(\mathrm{~b})$

(a) 20 for 2- or 3-story buildings.

(b) 14 for 3-story and 10 for 2-story buildings.

These values represent the level of general safety required by the 1973 Life Safety Code to house health care occupants in the class and height of building indicated. The analysis demonstrates that, in terms of the values in the evaluation system, the Life Safety Code minimum requirements are those for non-sprinklered buildings. The highest total value developed for a non-sprinklered building is 18. The importance of this value is that it was used as the approximate base line for the establishment of measurement of risk in a multi-story building and is the principal balance point for comparing occupancy risk with general safety. Thirteen is the comparable value for a single story building.

The values for existing buildings demonstrate the reduced level of general safety accepted by the Life Safety Code for these buildings. For a one-story building the general safety value is +13 for a new building and +5 for an existing building, indicating that for an existing one-story building only $38 \%$ of the score expected a new one-story building is required. Similarly, the ratio of existing multi-story buildings to that for new multi-story buildings is $1 / 2$. The 0.5 factor in table 3B of the Fire/Smoke Zone Evaluation Work Sheet reflects this ratio.

\subsection{Redundant Safety Subsystems}

A basic principal of the Life Safety Code is that there will be a redundancy of protection so that the failure of a single protection device or method will not result in a major failure of the entire safety system. In addition, the development of a redundant approach, as used in this safety evaluation system, avoids the pitfall of traditional approaches to developing grading systems where all of the elements are considered mutually exclusive of each other and a single total score determines acceptability. Under such a system, it is possible to completely disguise the absence of a critical element. The evaluation system establishes redundancy on the basis of in-depth coverage of the principal fire safety methodologies. The redundant methodologies used in the system are those related to fire safety through containment, through extinguishment and through people movement (including refuge). 
The redundant methodologies were chosen after examination of decision tree approaches $[1,2] .1$ These divide fire protection by element. Four different methodologies of managing fire impact were identified. These are control of the fuel and arrangement; compartmentation and other mechanisms of containment of the fire and its impact; extinguishment suppression and other means of terminating fire development; and the provision of safe locations of refuge either by evacuation or by establishment of safe areas of refuge. Those elements related to the control of fuel and its arrangement are incorporated into the risk analysis portion, in terms of the occupancy risk base line. Therefore, only three redundant methodologies were used in the analysis.

As part of the initial Delphi exercise, each member of the Delphi group completed a matrix establishing his judgment on the relative importance of the items in the general safety parameter matrix; he also made additional judgments on the same matrix elements related to the separate fire safety methodologies of containment, extinguishment, and people movement. These were then processed and analyzed and reviewed in subsequent conference meetings of the Delphi group. By this process, the parameters that have a significant impact on each of the redundant methodologies were identified. Many of the parameters impact on more than one of the methodologies. In the judgment of the group only sprinkler protection impacts on all three. Figure 19 shows the breakdown in terms of which parameters apply to which methodologies.

Each of these subsystems was then evaluated to determine the point value that would result from explicit compliance with the requirements of the Life Safety Code for that subsystem. Because of the variance between new and existing buildings and between single story and multistory-type buildings, four values were determined for each of the redundant safety methodologies. Figures 20, 21, and 22 demonstrate how these values were established.

In this fire safety evaluation system these values are mandatory minimum values that must be met regardless of the overall evaluated level of occupancy risk or the overall evaluated level of general safety.

\subsection{Overall Safety Evaluation of a Fire/Smoke Zone}

This evaluation system determines the efficacy of any arrangement of the listed fire safety subsystem elements in a fire/smoke zone by considering the risk factors and safety parameters relative to the level of safety that would be achieved by explicit conformance with the specific requirement of the Life Safety Code ((NFPA 101-1973). In order to be determined as equivalent the measurement must demonstrate that:

1. The general safety level produces a value that equals or exceeds the determined value or charge for risk.

INumbers in brackets refer to the references at the end of this paper. 
2. Each of the three individual redundancy groupings (containment safety; extinguishment safety; and people movement safety) must have an arrangement of safeguards that meet the prescribed minimum for that redundant grouping independent of the other condition.

\section{FIRE/SMOKE ZONE EVALUATION WORK SHEET FOR HEALTH CARE FACILITIES}

A11 of the parameters, variables and formulas for determining the facility safety equivalency with the Life Safety Code are contained in a self-instructing form. A separate manual (appendix D) has been prepared to assist in completion of the evaluation form. The manual provides expanded discussions and definitions of various items in the evaluation sheet to assist the surveyor or reviewer when questions of definitions, interpretations, or meanings arise. To evaluate totally a health care facility, it is necessary to evaluate each of the different fire/smoke zones. A zone is any space which is separated from all other spaces by floors, horizontal exits, or smoke barriers. Where a floor is not subdivided by smoke barriers, the entire floor is the zone. See figure 23 for an example of the form and appendix $E$ for examples of a completed form.

\section{COMPUTER ANALYSIS}

The evaluation system has a theoretical capability of evaluating about 230 million combinations of the 13 safety parameters and variations. Practical arrangements in existing buildings and future design are of the order of a few thousand. For the purpose of verifying the original proposed system as well as to analyze potential proposed changes, a computer program was developed. The program generates all arrangements that are valid, based on data given for the safety parameters. Using the Fire Safety Evaluation Work Sheet an experienced engineer or facility can manually analyze 10 to 15 differently organized zones, and establish the most suitable configuration for the facility. By using the computer program the evaluator is able to review all possible solutions to his problem, and he can also be assured that answers are not biased by individual preference. The printouts of the zone arrangements can be easily analyzed by an experienced individual to establish equivalency of solutions. Appendix $F$ provides additional detail on this program.

\section{EVALUATION SYSTEM ANALYSIS}

An effort has been made to make the system consistent with accepted and sound fire protection engineering practice and theory. While the most advanced scientific knowledge has been used in developing the system, the state-of-the-art requires that the insight and professional judgment of experts in the field serve as the base for information in developing the system, and the available technical knowledge (including results of fire tests, statistical analyses, fire investigations, etc.) 
serves a supportive role. In the development of the fire safety evaluation system, therefore, both the NBS Delphi group and the professional consulting panel described in sections 9.1 and 9.2 below were selected on the basis of expertise and interest.

In the systems proofing effort the capacity of these panels was broadened by using a two-step judgment approach. In the first step the relative worth of each parameter was individually judged on its relationship to other parameters. This evaluation was made in terms of each parameter's contribution to both general safety and to each of the redundant safety requirements. In the second phase the parameter values, developed in the preceding phase, were applied to a large number of test cases, scenarios and reviews of specific facilities. The resulting fire safety system configurations were then judged on overall equivalency to configurations prescribed by the 1973 Life Safety Code. This process provided a balance and cross check between judgments of the value of the individual parameters and evaluations of the overall product.

\subsection{NBS Delphi Group}

The initial safety parameter values (figure 3) were established by the NBS Delphi group. The group was composed of qualified fire protection engineers in the Fire Safety Engineering Division (see figure 24). The group was also used at different development stages to clarify technical problems and to analyze proposed changes suggested by the outside consultants, HEW or others.

Delphi is a procedure for obtaining the most reliable consensus of opinion of a group recognized as experts on a technical question for which no "true" answer is within the state of current knowledge [3]. The essence of the process is that the question is considered independently by members of the group. The response is tabulated and circulated to group members who revise their "answers" on the basis of further thought and consideration of the aggregate response. Additional rounds of response involving direct contact and discussion among the group members can ensue. The number of interactions is usually limited to four. In its classic form Delphi incorporates various statistical measures of the "convergence" to consensus, which are circulated within the group along with the responses. The Delphi exercise at NBS was in a form known as Policy Delphi. In this variant the statistical measures to establish convergence consensus are foregone and the "referee" or manager of the process takes a fairly active role in the discussion among the group members. In any of its formats, Delphi is widely perceived as furnishing useful information in areas in which questions are difficult to pose precisely, let alone answer definitely. A more detailed discussion of the Delphi operation is given in appendix A.

\subsection{Consultant Group}

After the NBS Delphi group agreed on an initial set of parameters and their values, the system was presented to the consultant group. 
The group consisted of prominent persons in the regulation or specification of fire safety for health care facilities. It included regulatory officials, code writing officials, government agency fire protection chiefs, and accrediting officials representing a cross section of the applied field. The membership and dates of meetings are shown in appendix B. This consultant group contributed to the development of the system and met in four separate sessions. In those meetings the consultants operated as a committee of the whole, reviewing the concept and individually discussing and evaluating the parameter values as developed by the NBS peer group. Important revisions resulted. Many of these revisions were in the form of restraints placed on the degree of liberality in the safety parameter values so as to require a more conservative and supportable approach.

\subsection{Inter-Group Relationships}

Throughout the project the project staff maintained liaison and a flow through the described review groups and a recently established task group of the National Fire Protection Association Committee on Safety to Life. This task group is studying the evaluation system for possible inclusion in the NFPA Life Safety Code. Figure 24 outlines this flow.

\section{SUMMARY}

The conclusions resulting from this study are briefly stated as follows:

a. A methodology has been developed and described for generating equivalency to a specified set of occupancy safety requirements. It is based on the understanding of level of occupancy risk, building safety and redundancy of safeguards. This methodology can provide the necessary flexibility for a designer to achieve minimum cost solutions for a specified level of safety.

b. The described methodology "System for Fire Safety Evaluation of Health Care Facilities," is a specific example of an equivalency approach. The system provides equivalency to the minimum life requirements for the health care facility as prescribed by Life Safety Code 101-1973. The system can be updated for later Life Safety Code editions for health care facilities.

c. Other equivalency systems can be developed for other occupancies but this will require detailed analysis of the risk level, the variety of building safety requirements, the necessary redundance equations, and other aspects of the specific occupancy involved. 


\section{REFERENCES}

[1] National Fire Protection Association, "Decision Tree," NFPA, Boston, 1974.

[2] Watts, J., "The Goal Oriented Systems Approach," NBS-GCR-77-103, National Bureau of Standards, Washington, D.C., July 12, 1977.

[3] Dalley, N. and Helmer, D., "An Experimental Application of the Delphi Method to the Use of Experts," Management Science 9, No. 3, p. 458 (April 1963). 


\begin{tabular}{|c|c|c|c|c|c|c|}
\hline \multicolumn{7}{|c|}{ OCCUPANCY RISK PARAMETER FAC } \\
\hline RISK PARAMETERS & \multicolumn{6}{|c|}{ RISK FACTOR VALUES } \\
\hline \multirow{2}{*}{$\begin{array}{l}\text { 1. PATIENT } \\
\text { MOBILITY (M) }\end{array}$} & $\begin{array}{l}\text { MOBILITY } \\
\text { STATUS }\end{array}$ & MOBILE & \multicolumn{2}{|c|}{$\begin{array}{l}\text { LIMITED } \\
\text { MOBILITY }\end{array}$} & \multicolumn{2}{|c|}{$\begin{array}{l}\text { NOT } \\
\text { MOVABLE }\end{array}$} \\
\hline & RISK FACTOR & 1.0 & \multicolumn{2}{|l|}{1.6} & \multicolumn{2}{|c|}{4.5} \\
\hline \multirow{2}{*}{$\begin{array}{l}\text { 2. PATIENT } \\
\text { DENSITY }(D)\end{array}$} & PATIENT & 1.5 & $6 \cdot 10$ & $11 \cdot 30$ & $>30$ & \\
\hline & RISK FACTOR & 1.0 & 1.2 & 1.5 & 2.0 & \\
\hline \multirow{2}{*}{$\begin{array}{l}\text { 3. ZONE } \\
\text { LOCATION (L) }\end{array}$} & FLOOR & $1 S T$ & $\begin{array}{c}2 \mathrm{NDOR} \\
3 \mathrm{RD}\end{array}$ & $\begin{array}{l}4 \text { TTH TO } \\
\text { 6TH }\end{array}$ & $\begin{array}{l}\text { 7TH AND } \\
\text { ABOVE }\end{array}$ & $\begin{array}{l}\text { BASE- } \\
\text { MENTS }\end{array}$ \\
\hline & RISK FACTOR & 1.1 & 1.2 & 1.4 & 1.6 & 1.6 \\
\hline \multirow{3}{*}{$\begin{array}{l}\text { 4. RATIO OF } \\
\text { PATIENTS TO } \\
\text { ATTENDANTS }(T)\end{array}$} & PATIENTS & $1-2$ & 3.5 & $\underline{6-10}$ & $>11$ & $\begin{array}{l}\text { ONE OR* } \\
\text { MORE }\end{array}$ \\
\hline & ATTENDANT & 1 & 1 & 1 & 1 & $\overline{\text { NONE }}$ \\
\hline & RISK FACIIOR & 1.0 & 1.1 & 1.2 & 1.5 & 4.0 \\
\hline \multirow{2}{*}{$\begin{array}{l}\text { 5. PATIENT } \\
\text { AVERAGE } \\
\text { AGE }(A)\end{array}$} & AGE & \multicolumn{2}{|c|}{$\begin{array}{l}\text { UNDER } 65 \text { YEARS } \\
\text { AND OVER } 1 \text { YEAR }\end{array}$} & \multicolumn{3}{|c|}{$\begin{array}{l}65 \text { YEARS \& OVER } \\
1 \text { YEAR \& YOUNGER }\end{array}$} \\
\hline & RISK FACTOR & \multicolumn{2}{|c|}{1.0} & \multicolumn{2}{|c|}{1.2} & \\
\hline \multicolumn{7}{|c|}{$\begin{array}{l}\text { * RISK FACTOR OF } 4.0 \text { IS CHARGED TO ANY ZONE THAT HOUSES } \\
\text { PATIENTS WITHOUT ANY STAFF IN IMMEDIATE ATTENDANCE }\end{array}$} \\
\hline
\end{tabular}

Figure 1. Occupancy risk parameter factors 


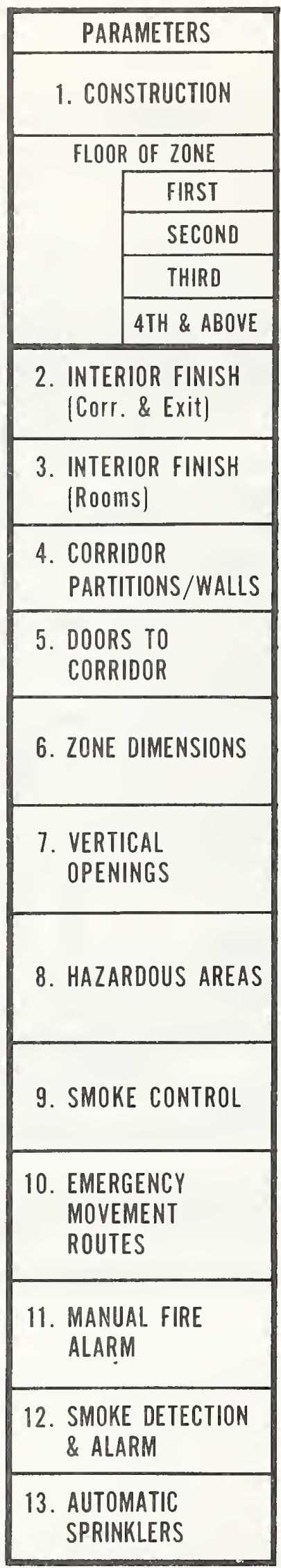

Figure 2. Safety parameters 


\begin{tabular}{|c|c|c|c|c|c|c|c|}
\hline \multicolumn{8}{|c|}{ SAFETY PARAMETERS VALUES } \\
\hline PARAMETERS & \multicolumn{7}{|c|}{$\begin{array}{l}\text { PARAMETERS VALUES } \\
\end{array}$} \\
\hline \multirow{2}{*}{ 1. CONSTRUCTION } & \multicolumn{4}{|c|}{ COMBUSTIBLE } & \multirow{2}{*}{\multicolumn{3}{|c|}{ NON-COMBUSTIBLE }} \\
\hline & \multicolumn{2}{|c|}{ WOOO FRAME } & \multicolumn{2}{|c|}{ ORDINARY } & & & \\
\hline FLOOR OF ZONE & \begin{tabular}{|l|l|} 
UNPROTECTED & PRO \\
\end{tabular} & PROTECTED & \multirow{2}{*}{\begin{tabular}{|c|} 
UNPROTECTEO \\
-2
\end{tabular}} & \multirow{2}{*}{\begin{tabular}{|c|} 
PROTECTEO \\
0 \\
\end{tabular}} & \multirow{2}{*}{$\begin{array}{c}\text { UNPROTECTED } \\
0 \\
\end{array}$} & \multirow{2}{*}{\begin{tabular}{|c|} 
PROTECTED \\
2 \\
\end{tabular}} & FIRE RESIST. \\
\hline FIRST & \begin{tabular}{l|l}
-2 & \\
\end{tabular} & 0 & & & & & 2 \\
\hline SECOND & -7 & -2 & -4 & -2 & -2 & 2 & 4 \\
\hline THIRD & -9 & -7 & -9 & -7 & -7 & 2 & 4 \\
\hline 4TH \& ABOVE & -13 & -7 & -13 & -7 & -9 & -7 & 4 \\
\hline \multirow{2}{*}{$\begin{array}{l}\text { 2. INTERIOR FINISH } \\
\text { (Corr. \& Exit) }\end{array}$} & CLASS C & \multicolumn{2}{|c|}{ CLASS B } & CLASS A & & & \\
\hline & -5 & \multicolumn{2}{|r|}{0} & 3 & & & \\
\hline \multirow{2}{*}{$\begin{array}{l}\text { 3. INTERIOR FINISH } \\
\text { (ROOms) }\end{array}$} & CLASS C & \multicolumn{2}{|c|}{ CLASS B } & CLASS A & & & \\
\hline & -3 & & 1 & 3 & & & \\
\hline 4. CORRIDOR & $\begin{array}{c}\text { NONE OR } \\
\text { INCOMPLETE }\end{array}$ & & $1 / 3 \mathrm{HR}$ & $>1 / 3<1.0 \mathrm{HR}$ & $\geqslant 1.0$ & & \\
\hline PARTITIONS/WALLS & $-10(0)^{*}$ & & 0 & $1|0| *$ & 210 & )$^{*}$ & \\
\hline 5. DOORS TO & NO OOOR & & MIN.FR & $>20$ MIN FR & $\begin{array}{l}>20 \text { MIN. } \\
\text { AUTD }\end{array}$ & $\begin{array}{l}\text { FR\& } \\
\text { LOS }\end{array}$ & \\
\hline CORRIDOR & -10 & & 0 & $1(0)^{\cdots \cdots}$ & 210 & )$^{\infty \cdots \bullet}$ & \\
\hline & $\begin{array}{c}\text { OEAD END } \\
\text { MORE THAN } 100^{\circ}\end{array}$ & & $\begin{array}{l}A D E N O \\
0^{\prime}-100^{\circ}\end{array}$ & NO DEAD & ENDS $>30^{\prime} \&$ & ZONE LEN & GTH IS: \\
\hline 6. ZONE DIMENSIONS & & & & $>150^{\circ}$ & $100^{\circ}-$ & & $<100^{\circ}$ \\
\hline & $-6(0) * *$ & & $4(0)^{* *}$ & -2 & 0 & & 1 \\
\hline & OPEN 4 OR MORE & & $N 2 O R 3$ & ENCLOSE & ED WITH INDI & CATED FIR & RESIST \\
\hline 7. VERTICAL & FLOORS & & LOORS & $<1 \mathrm{HR}$ & $\geqslant 1 \mathrm{HR}$.< & \begin{tabular}{|l|l}
$2 \mathrm{HR}$ & \\
\end{tabular} & $\geqslant 2 \mathrm{HR}$ \\
\hline & -14 & & -10 & 0 & 210 & )$^{\bullet}$ & $3(0)^{\bullet}$ \\
\hline & DOUBLE D & DEFICIENC & & SINGLE 0 & OEFICIENCY & & NO DEFICIENCIES \\
\hline 0 HUTAODOULC ADFAC & IN 20NE & \begin{tabular}{l|l} 
OUT \\
\end{tabular} & SIOE ZDNE & IN ZONE & IN ADJACEI & TI ZONE & 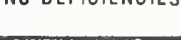 \\
\hline 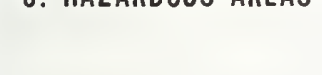 & -11 & & -5 & -6 & -2 & & 0 \\
\hline & NO CONTRDL & SMOK & PARTITION & MECH. ASSIS & STED SYSTEM & & \\
\hline 9. SMOKE CONTROL & & & & BY ZONE & BY COR & RIDOR & \\
\hline & $-2(0) * * *$ & & 0 & 3 & 4 & & \\
\hline & $\angle 2$ ROUTES & & & MULTIPI & LE ROUTES & & \\
\hline $\begin{array}{l}\text { 10. EMERLENCY } \\
\text { MOVEMENT }\end{array}$ & & & $\begin{array}{l}\text { FICIENT } \\
\text { PACITY }\end{array}$ & $\begin{array}{c}\begin{array}{c}\text { W/0 HORIZONTAL } \\
\text { EXITIS] }\end{array} \\
\end{array}$ & $\begin{array}{ll}\text { HORIZONTA } \\
\end{array}$ & LEXIT|s] & DIRECT EXIT/S| \\
\hline ROUTES & -8 & & -2 & 0 & 3 & & 5 \\
\hline & NO MANUAL & AL FIRE AL & IARM & MANUAL & FIRE ALARM & & \\
\hline 11. MANUAL FIRE & & & & W/D F.O. CONN & W/F.0.0 & ONN. & \\
\hline & & -4 & & 1 & 2 & & \\
\hline 12. SMOKE DETECTION & NONE & CORF & RIOOR ONLY & ROOMS ONLY & CORRID & OR \& & TDTAL SPACE \\
\hline \& ALARM & 0 & & 2 & 3 & 4 & & 5 \\
\hline 13. AUTOMATIC & NONE & & ORRIOOR & $\begin{array}{l}\text { CORRIOOR\& } \\
\text { HABIT. SPACE }\end{array}$ & TOTAL & PACE & \\
\hline SPRINKLERS & 0 & & $2|0|^{\circ \bullet}$ & 8 & 10 & & \\
\hline
\end{tabular}

Figure 3. Safety parameters values 


\begin{tabular}{|c|c|c|c|c|}
\hline $\begin{array}{c}\text { SAFETY } \\
\text { PARAMETERS }\end{array}$ & NEW - 1 STORY & $\begin{array}{l}\text { NEW - MORE } \\
\text { THAN } 1 \text { STORY }\end{array}$ & $\begin{array}{l}\text { EXISTING - } \\
1 \text { STORY }\end{array}$ & $\begin{array}{l}\text { EXISTING - } \\
\text { MORE THAN } \\
1 \text { STORY }\end{array}$ \\
\hline \multirow{2}{*}{ 1. CONSTRUCTION } & PROT. NON COMB. & FIRE RESISTIVE & PROT. NON COMB. & FIRE RESISTIVE \\
\hline & 2 & 4 & 2 & 4 \\
\hline \multirow{2}{*}{$\begin{array}{l}\text { 2. INTERIOR FINISH } \\
\text { (Corr. \& Exit) }\end{array}$} & CLASS A & CLASS A & CLASS B & CLASS B \\
\hline & 3 & 3 & 0 & 0 \\
\hline \multirow{2}{*}{$\begin{array}{l}\text { 3. INTERIOR FINISH } \\
\text { (Rooms) }\end{array}$} & CLASS B & CLASS B & CLASS B & CLASS B \\
\hline & 1 & 1 & 1 & 1 \\
\hline \multirow{2}{*}{$\begin{array}{l}\text { 4. CORRIDOR } \\
\text { PARTITIONS/WALLS }\end{array}$} & $\geqslant 1.0 \mathrm{HR}$ & $\geqslant 1.0 \mathrm{HR}$ & $<1 / 3 \mathrm{HR}$ & $<1 / 3 \mathrm{HR}$ \\
\hline & 2 & 2 & 0 & 0 \\
\hline \multirow{2}{*}{$\begin{array}{l}\text { 5. DOORS TO } \\
\text { CORRIDOR }\end{array}$} & $\geqslant 20$ MIN. & $\geqslant 20$ MIN. & $\geqslant 20$ MIN. & $\geq 20 \mathrm{MIN}$ \\
\hline & 1 & 1 & 1 & 1 \\
\hline \multirow{2}{*}{ 6. ZONE DIMENSIONS } & $100^{\prime}-150^{\prime}$ & $100^{\prime}-150^{\prime}$ & $100^{\prime}-150^{\prime}$ & $100^{\prime}-150^{\prime}$ \\
\hline & 0 & 0 & 0 & 0 \\
\hline \multirow{2}{*}{ 7. VERTICAL OPENINGS } & NON APP. & $2 \mathrm{HR}$ & NON APP. & $\geqslant 1-<2 \mathrm{HR}$ \\
\hline & $\begin{array}{l}0 \\
\end{array}$ & 3 & 0 & 2 \\
\hline \multirow{2}{*}{ 8. HAZARDOUS AREAS } & NO DEFICIENCIES & NO DEFICIENCIES & NO DEFICIENCIES & NO DEFICIENCIES \\
\hline & 0 & 0 & 0 & 0 \\
\hline \multirow{2}{*}{ 9. SMOKE CONTROL } & SMOKE PART. & SMOKE PART. & SMOKE PART. & SMOKE PART. \\
\hline & 0 & 0 & 0 & 0 \\
\hline \multirow{2}{*}{$\begin{array}{l}\text { 10. EMERGENCY } \\
\text { MOVEMENT ROUTES }\end{array}$} & MULTIPLE ROUTES & MULTIPLE ROUTES & MULTIPLE ROUTES & MULTIPLE ROUTES \\
\hline & 0 & 0 & 0 & 0 \\
\hline \multirow{2}{*}{$\begin{array}{l}\text { 11. MANUAL FIRE } \\
\text { ALARM }\end{array}$} & W/FD CONN. & W/FD CONN. & W/O/FO CONN. & W/O/FD CONN. \\
\hline & 2 & 2 & 1 & 1 \\
\hline \multirow{2}{*}{$\begin{array}{l}\text { 12. SMOKE DETECTION } \\
\& \text { ALARM }\end{array}$} & CORRIDOR ONLY & CORRIDOR ONLY & NONE & NONE \\
\hline & 2 & 2 & 0 & 0 \\
\hline \multirow{2}{*}{$\begin{array}{l}\text { 13. AUTOMATIC } \\
\text { SPRINKLERS }\end{array}$} & NONE & NONE & NONE & NONE \\
\hline & 0 & 0 & 0 & 0 \\
\hline TOTAL VALUE & 13 & 18 & 5 & 9 \\
\hline
\end{tabular}

Figure 4. Life safety code requirements (non-sprinklered) 


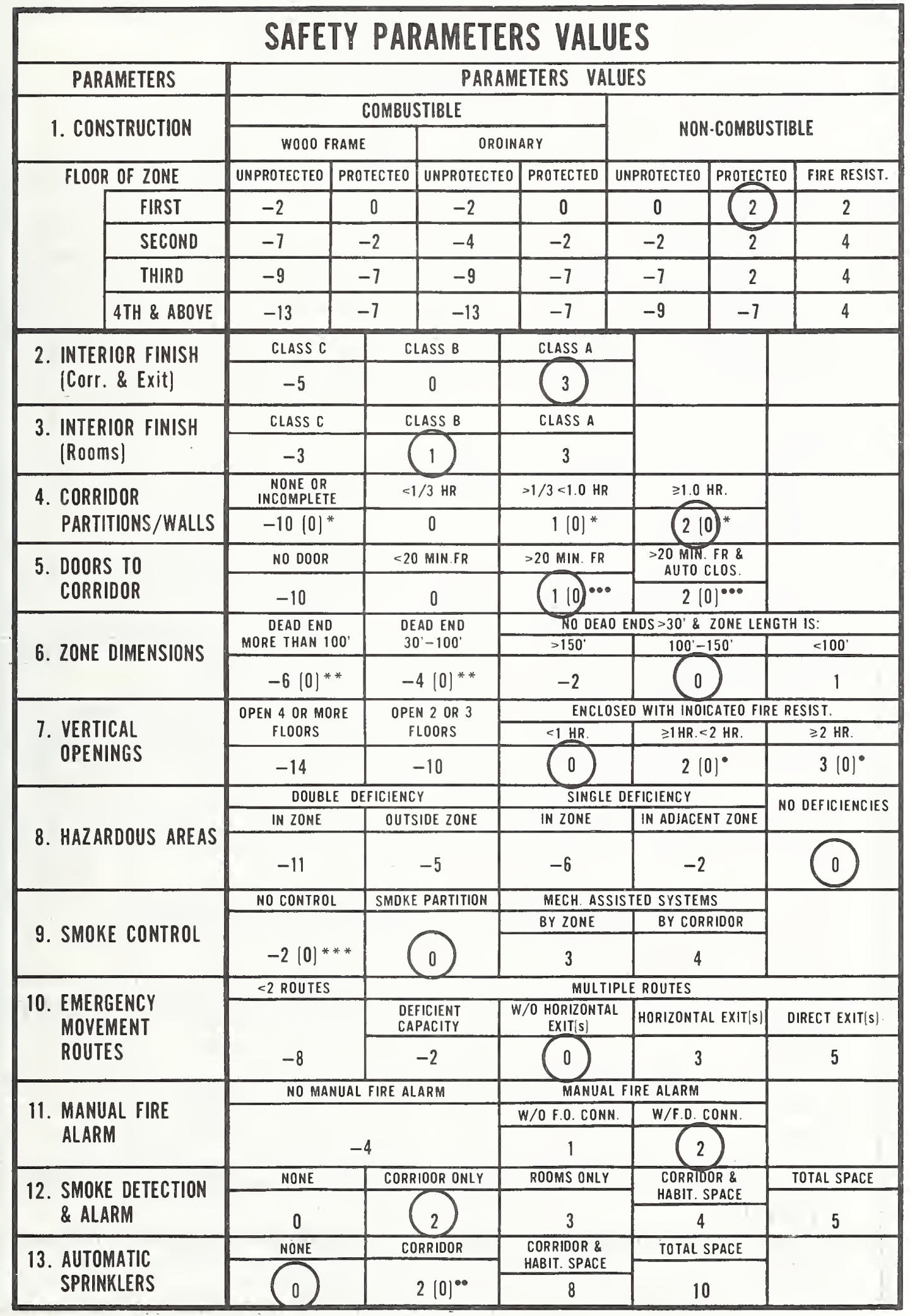

Figure 5. Life safety code requirements - new, non-sprinklered, one story facility 


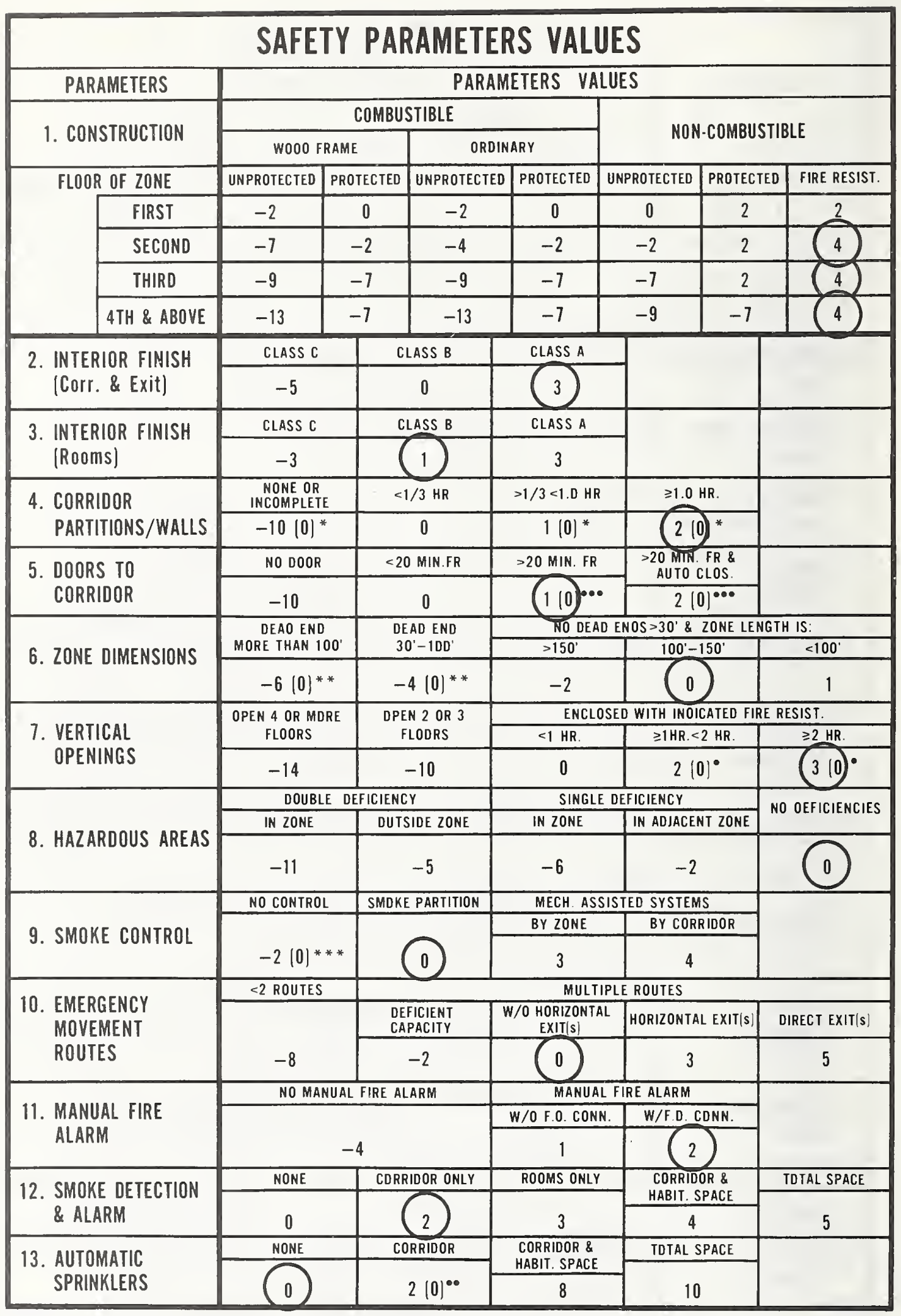

Figure 6. Life safety code requirements - new, non-sprinklered, more than one story facility 


\begin{tabular}{|c|c|c|c|c|c|c|c|c|}
\hline PARAMETERS & \multicolumn{8}{|c|}{ PARAMETERS VALUES } \\
\hline \multirow{2}{*}{ 1. CONSTRUCTION } & \multicolumn{4}{|c|}{ COMBUSTIBLE } & \multirow{2}{*}{\multicolumn{4}{|c|}{ NON-COMBUSTIBLE }} \\
\hline & \multicolumn{2}{|c|}{ WOOD FRAME } & \multicolumn{2}{|c|}{ ORDINARY } & & & & \\
\hline FLOOR OF ZONE & \begin{tabular}{|l|l|} 
UNPROTECTED & PI \\
\end{tabular} & PROTECTED & UNPROTECTED & \begin{tabular}{|l|l|} 
PROTECTED & 1 \\
\end{tabular} & \multirow{2}{*}{$\begin{array}{c}\text { UNPROTECTED } \\
0\end{array}$} & \multirow{2}{*}{\multicolumn{2}{|c|}{$\overbrace{(2)}^{\text {PROTECTED }}$}} & \multirow{2}{*}{\begin{tabular}{|c|} 
FIRE RESIST \\
2 \\
\end{tabular}} \\
\hline FIRST & \begin{tabular}{l|l}
-2 & \\
\end{tabular} & 0 & -2 & \begin{tabular}{l|l}
0 & \\
\end{tabular} & & & & \\
\hline SECOND & -7 & -2 & -4 & -2 & -2 & 2 & & 4 \\
\hline THIRD & -9 & -7 & -9 & -7 & -7 & 2 & & 4 \\
\hline 4TH \& ABOVE & -13 & -7 & -13 & -7 & -9 & -7 & & 4 \\
\hline \multirow{2}{*}{$\begin{array}{l}\text { 2. INTERIOR FINISH } \\
\text { (Corr. \& Exit) }\end{array}$} & CLASS C & \multicolumn{2}{|c|}{ CLASS B } & CLASS A & & & & \\
\hline & -5 & & $0)$ & 3 & & & & \\
\hline \multirow{2}{*}{$\begin{array}{l}\text { 3. INTERIOR FINISH } \\
\text { (ROOMS) }\end{array}$} & CLASS C & \multicolumn{2}{|c|}{ CLASS B } & CLASS A & & & & \\
\hline & -3 & & 1) & 3 & & & & \\
\hline \multirow{2}{*}{$\begin{array}{l}\text { 4. CORRIDOR } \\
\text { PARTITIONS/WALLS }\end{array}$} & $\begin{array}{c}\text { NONE DR } \\
\text { INCOMPLETE }\end{array}$ & & $/ 3 \mathrm{HR}$ & $>1 / 3<1.0 \mathrm{HR}$ & $\geqslant 1.0$ & & & \\
\hline & $-10(0)^{*}$ & & $0)$ & $1(0)^{*}$ & $2[0$ & )$^{*}$ & & \\
\hline 5. DOORS TO & NO DOOR & & MIN.FR & $>20 \mathrm{MIN} \mathrm{FR}$ & $\begin{array}{l}>20 \text { MIN. } \\
\text { AUTO }\end{array}$ & $\begin{array}{l}\text { FR \& } \\
\text { LOS }\end{array}$ & & \\
\hline CORRIDOR & -10 & & 0 & $1|d|^{\bullet \bullet}$ & & ]$^{\bullet \bullet \bullet}$ & & \\
\hline & DEAD END & & AD END & NO DEAD & ENDS $>30^{\prime} \&$ & ZONE LEN & GTH & \\
\hline 6. ZONE DIMENSIONS & MORE THAN $100^{\circ}$ & & $y^{\prime}-100^{\prime}$ & $2150^{\circ}$ & \begin{tabular}{l|l}
$100^{\prime}-$ \\
\end{tabular} & & & $<100^{\prime}$ \\
\hline & $-6(0) * *$ & & $4(0)^{* *}$ & -2 & 10 & ) & & 1 \\
\hline & OPEN 4 OR MORE & & N 2 DR 3 & ENCLOS & SEO WITH INOI & CATED FIR & ERES & \\
\hline 7. VERTICAL & FLDDRS & & LDORS & $<1 \mathrm{HR}$ & \begin{tabular}{l|l}
$\mathrm{HR} .<$ \\
\end{tabular} & \begin{tabular}{|l|l}
$2 \mathrm{HR}$ \\
\end{tabular} & & $\geqslant 2 \mathrm{HR}$. \\
\hline & -14 & & -10 & $0)$ & $2 \mid 0$ & & & $3|0|^{\circ}$ \\
\hline & DOUBLE & DEFICIENC & & SINGLE & DEFICIENCY & & & DEFICIENCIES \\
\hline 8 HA7ARONUS ARFAS & IN ZONE & \begin{tabular}{l|l} 
& $0 U T S$ \\
\end{tabular} & IDE ZDNE & IN ZDNE & IN ADJACEI & IT ZONE & & 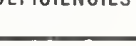 \\
\hline & -11 & & -5 & -6 & -2 & & & 0 \\
\hline & NO CDNTRDL & SMOKE & PARTITIDN & MECH. ASSI & ISTED SYSTEM & & & \\
\hline 9 SMOKE CONTROL & & & & BY ZONE & \begin{tabular}{l|l} 
BY CDR \\
\end{tabular} & RIDOR & & \\
\hline & $-2(0) * * *$ & & & 3 & 4 & & & \\
\hline & $<2$ ROUTES & & & MULTIP & PLE ROUTES & & & \\
\hline $\begin{array}{l}\text { TU. EMERGENCY } \\
\text { MOVEMENT }\end{array}$ & & & $\begin{array}{l}\text { FICIENT } \\
\text { PACITY } \\
\end{array}$ & $\begin{array}{c}\text { W/0 HORIZONTAL } \\
\text { EXIIIs) }\end{array}$ & HDRIZONTA & L EXIT/s| & & (ECT EXIT|S\} \\
\hline ROUTES & -8 & & -2 & (0) & 3 & & & 5 \\
\hline & ND MANU & UAL FIRE AL & ARM & MANUAL & FIRE ALARM & & & \\
\hline 11. MANUAL FIRE & & & & W/O F.D. CONN & W/F.D. C & $0 N \mathrm{NN}$ & & \\
\hline & & -4 & & 1) & 2 & & & \\
\hline 12. SMOKE DETECTION & NONE & CORP & IIOOR ONLY & RDOMS ONLY & $\begin{array}{l}\text { CORRIDI } \\
\text { HABIT. S }\end{array}$ & $\begin{array}{l}\mathrm{DR} \& \\
\text { PACE }\end{array}$ & & TAL SPACE \\
\hline \& ALARM & $0)$ & & 2 & 3 & 4 & & & 5 \\
\hline 13. AUTOMATIC & NONE & & RRIDOR & $\begin{array}{l}\text { CORRIDOR \& } \\
\text { HABIT. SPACE }\end{array}$ & TOTAL S & PACE & & \\
\hline SPRINKLERS & 0 & & $2[0]^{\bullet \bullet}$ & 8 & 10 & & & \\
\hline
\end{tabular}

Figure 7. Life safety code requirements - existing, non-sprinklered one story facility 


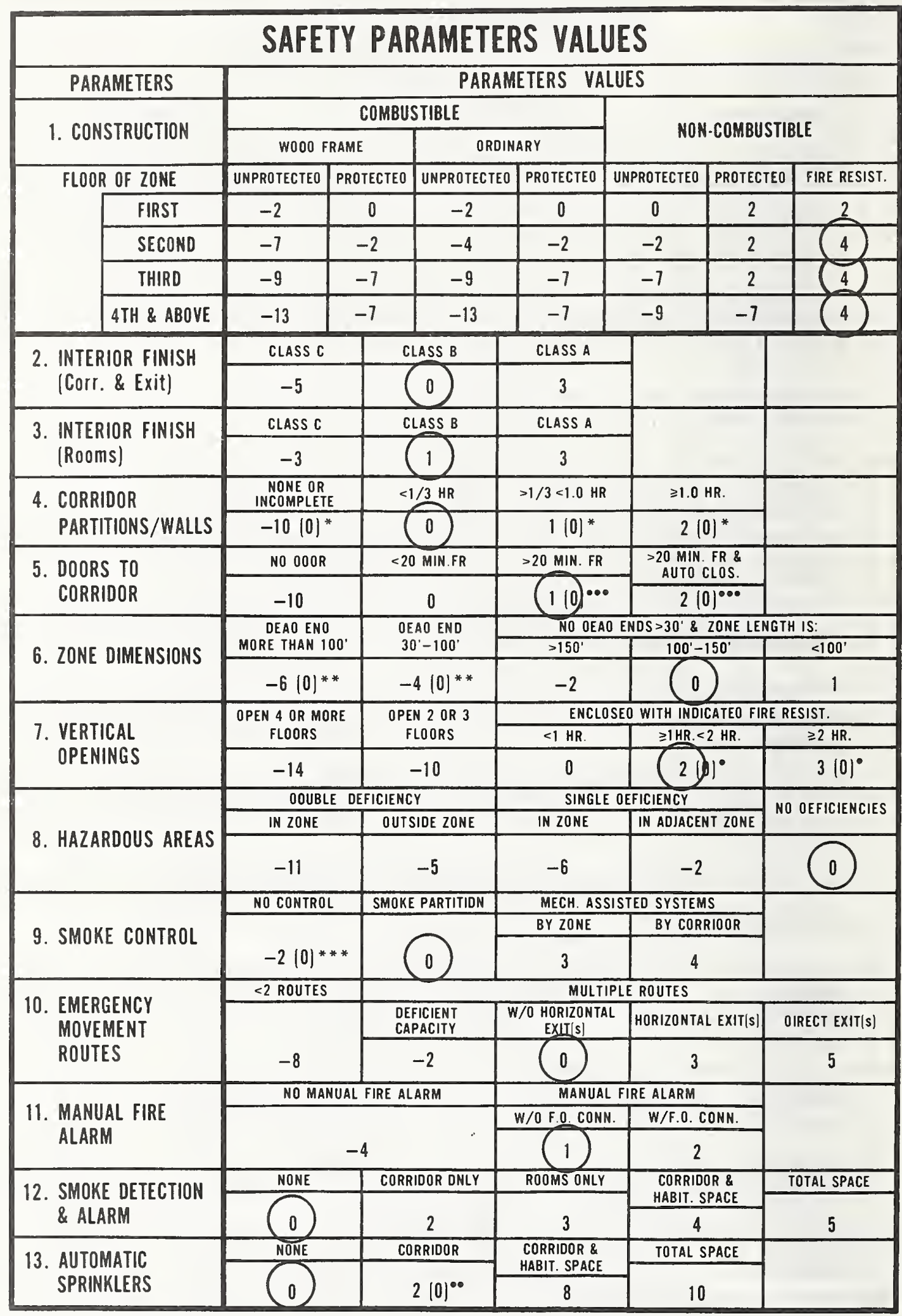

Figure 8. Life safety code requirements - existing, non-sprinklered more, than one story facility 


\begin{tabular}{|c|c|c|c|c|}
\hline $\begin{array}{c}\text { SAFETY } \\
\text { PARAMETERS }\end{array}$ & NEW - 1 STORY & $\begin{array}{l}\text { NEW }- \text { MORE } \\
\text { THAN } 1 \text { STORY }\end{array}$ & $\begin{array}{l}\text { EXISTING - } \\
1 \text { STORY }\end{array}$ & $\begin{array}{l}\text { EXISTING - } \\
\text { MORE THAN } \\
1 \text { STORY }\end{array}$ \\
\hline \multirow{2}{*}{ 1. CONSTRUCTION } & PROTECT. COMB. & FIRE RESISTIVE & UNPROT. COMB. & FIRE RESISTIVE \\
\hline & 0 & 4 & -2 & $4 *$ \\
\hline \multirow{2}{*}{$\begin{array}{l}\text { 2. INTERIOR FINISH } \\
\text { (Corr. \& Exit) }\end{array}$} & CLASS A & CLASS A & CLASS B & CLASS B \\
\hline & 3 & 3 & 0 & 0 \\
\hline \multirow{2}{*}{$\begin{array}{l}\text { 3. INTERIOR FINISH } \\
\text { (Rooms) }\end{array}$} & CLASS B & CLASS B & CLASS B & CLASS B \\
\hline & 1 & 1 & 1 & 1 \\
\hline \multirow{2}{*}{$\begin{array}{l}\text { 4. CORRIDOR } \\
\text { PARTITIONS/WALLS }\end{array}$} & $<1 / 3 \mathrm{HR}$ & $<1 / 3 \mathrm{HR}$ & $<1 / 3 \mathrm{HR}$ & $\angle 1 / 3 \mathrm{HR}$ \\
\hline & 0 & 0 & 0 & 0 \\
\hline \multirow{2}{*}{$\begin{array}{l}\text { 5. DOORS TO } \\
\text { CORRIDOR }\end{array}$} & $<20$ MIN. & $<20 \mathrm{MIN}$. & $<20$ MIN. & $<20$ MIN. \\
\hline & 0 & 0 & 0 & 0 \\
\hline 6. ZONE DIMENSIONS & $\begin{array}{l}>150^{\prime} \\
-2\end{array}$ & $\frac{150^{\prime}}{-2}$ & $\begin{array}{l}>150^{\prime} \\
-2\end{array}$ & $\frac{>150^{\prime}}{-2}$ \\
\hline \multirow{2}{*}{ 7. VERTICAL OPENINGS } & NOT APP. & $2 \mathrm{HR}$ & NOT APP. & $\geqslant 1-<2 \mathrm{HR}$ \\
\hline & 0 & 3 & 0 & 2 \\
\hline \multirow{2}{*}{ 8. HAZARDOUS AREAS } & NO DEFICIENCIES & NO DEFICIENCIES & NO DEFICIENCIES & NO DEFICIENCIES \\
\hline & $\frac{0}{\text { SMOKE PART. }}$ & 0 & 0 & 0 \\
\hline 9. SMOKE CONTROL & $\frac{\text { SMOKE PART. }}{0}$ & SMOKE PART. & $\begin{array}{l}\text { SMOKE PART. } \\
0\end{array}$ & SMOKE PART. \\
\hline \multirow{2}{*}{$\begin{array}{l}\text { 10. EMERGENCY } \\
\text { MOVEMENT ROUTES }\end{array}$} & MULTIPLE ROUTES & MULTIPLE ROUTES & MULTIPLE ROUTES & MULTIPLE ROUTES \\
\hline & 0 & 0 & 0 & 0 \\
\hline \multirow{2}{*}{$\begin{array}{l}\text { 11. MANUAL FIRE } \\
\text { ALARM }\end{array}$} & W/FD CONN. & W/FD CONN. & W/O/FD CONN. & W/O/FD CONN. \\
\hline & 2 & 2 & 1 & 1 \\
\hline \multirow{2}{*}{$\begin{array}{l}\text { 12. SMOKE DETECTION } \\
\text { \& ALARM }\end{array}$} & CORRIDOR ONLY & CORRIDOR ONLY & NONE & NONE \\
\hline & 2 & 2 & 0 & 0 \\
\hline \multirow{2}{*}{$\begin{array}{l}\text { 13. AUTOMATIC } \\
\text { SPRINKLERS }\end{array}$} & TOTAL SPACE & TOTAL SPACE & TOTAL SPACE & TOTAL SPACE \\
\hline & 10 & 10 & 10 & 10 \\
\hline TOTAL VALUE & 16 & 23 & 8 & 16 \\
\hline
\end{tabular}

* Sec figure Il for 2 ant 3 stony 3allingss.

Figure 9. Life safety code requirements (sprinklered) 


\begin{tabular}{|c|c|c|c|c|c|c|c|}
\hline \multicolumn{8}{|c|}{ SAFETY PARAMETERS VALUES } \\
\hline PARAMETERS & \multicolumn{7}{|c|}{ PARAMETERS VALUES } \\
\hline \multirow{2}{*}{ 1. CONSTRUCTION } & \multicolumn{4}{|c|}{ COMBUSTIBLE } & \multirow{2}{*}{\multicolumn{3}{|c|}{ NON-COMBUSTIBLE }} \\
\hline & \multicolumn{2}{|c|}{ WOOD FRAME } & \multicolumn{2}{|c|}{ ORDINARY } & & & \\
\hline FLOOR OF ZONE & \begin{tabular}{|l|l|} 
UNPROTECTEO & PF \\
\end{tabular} & PROTECTED & UNPROTECTEO & PROTECTED U & \multirow{2}{*}{\begin{tabular}{|c|} 
UNPROTECTEO \\
0 \\
\end{tabular}} & \multirow{2}{*}{\begin{tabular}{|c|} 
PROTECTEO \\
2 \\
\end{tabular}} & \multirow{2}{*}{\begin{tabular}{|c|} 
FIRE RESIST. \\
2 \\
\end{tabular}} \\
\hline FIRST & \begin{tabular}{|l|l|}
-2 & \\
\end{tabular} & $(0)$ & -2 & $(0)$ & & & \\
\hline SECOND & -7 & -2 & -4 & -2 & -2 & 2 & 4 \\
\hline THIRD & -9 & -7 & -9 & -7 & -7 & 2 & 4 \\
\hline 4TH \& ABOVE & -13 & -7 & -13 & -7 & -9 & -7 & 4 \\
\hline \multirow{2}{*}{$\begin{array}{l}\text { 2. INTERIOR FINISH } \\
\text { (Corr. \& Exit) }\end{array}$} & CLASS C & \multicolumn{2}{|c|}{ CLASS B } & CLASS A & & & \\
\hline & -5 & & 0 & (3) & & & \\
\hline \multirow{2}{*}{$\begin{array}{l}\text { 3. INTERIOR FINISH } \\
\text { (ROOMS) }\end{array}$} & CLASS C & \multicolumn{2}{|c|}{ CLASS B } & CLASS A & & & \\
\hline & -3 & & 1) & 3 & & & \\
\hline \multirow{2}{*}{$\begin{array}{l}\text { 4. CORRIDOR } \\
\text { PARTITIONS/WALLS }\end{array}$} & $\begin{array}{c}\text { NONE OR } \\
\text { INCOMPLETE } \\
\end{array}$ & \multicolumn{2}{|c|}{$<1 / 3 \mathrm{HR}$} & $>1 / 3<1.0 \mathrm{HR}$ & $\geqslant 1.0 \mathrm{r}$ & & \\
\hline & $-10(0)^{*}$ & & $0)$ & $1(0)^{*}$ & 210 & $0)^{*}$ & \\
\hline 5. DOORS TO & N0 000R & & MIN.FR & $>20$ MIN. FR & $\begin{array}{l}>20 \text { MIN. } \\
\text { AUTO C }\end{array}$ & $\begin{array}{l}\text { FR\& } \\
\text { CLOS. }\end{array}$ & \\
\hline CORRIDOR & -10 & & $0)$ & $1(0)^{000}$ & 210 & $0)^{\bullet \bullet \bullet}$ & \\
\hline & $\begin{array}{l}\text { OEAO ENO } \\
\text { MORE THAN } 100^{\prime}\end{array}$ & & AO ENO & $\begin{array}{l}\text { NO OEAO } \\
>150^{\prime}\end{array}$ & $\frac{100^{\prime}-1}{\text { ENOS }^{\prime}>30^{\prime} \&}$ & $\begin{array}{ll}\text { ZONE LEN } \\
150^{\prime}\end{array}$ & $\frac{\text { GTH IS: }}{<100^{\prime}}$ \\
\hline O. LUNE DIMENASIUNS & $-6(0)^{* *}$ & & $4(0)^{* *}$ & (-2) & 0 & & 1 \\
\hline & OPEN 4 OR MORE & & N 2 OR 3 & ENCLOSE & SEO WITH INOIC & CATEO FIR & RESIST. \\
\hline 7. VERTICAL & FLOORS & & L00RS & $<1 \mathrm{HR}$. & \begin{tabular}{l|l} 
& $\geqslant 1$ HR.< \\
\end{tabular} & $2 \mathrm{HR}$. & $\geqslant 2 \mathrm{HR}$. \\
\hline & -14 & & -10 & $(0)$ & 210 & & $3(0)^{\bullet}$ \\
\hline & OOUBLE & OEFICIENC & & SINGLE 0 & OEFICIENCY & & NO OEFICIENCIES \\
\hline 8 HAZAROOUIS ARFAS & IN ZONE & \begin{tabular}{l|l} 
OUTS \\
\end{tabular} & IOE ZONE & IN ZONE & \begin{tabular}{l|l} 
IN AOJACEN \\
\end{tabular} & NT ZONE & 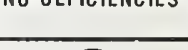 \\
\hline & -11 & & -5 & -6 & -2 & & \\
\hline & NO CONTROL & SMOKE & PARTITION & MECH. ASSIS & ISTEO SYSTEM & & \\
\hline 9. SMOKE CONTROL & & & & BY ZONE & \begin{tabular}{l|l} 
BY CORF \\
\end{tabular} & RIDOR & \\
\hline & $-2(0) * * *$ & & & 3 & 4 & & \\
\hline & $<2$ ROUTES & & & MULTIPL & PLE ROUTES & & \\
\hline $\begin{array}{l}\text { 10. EMIERLENLY } \\
\text { MOVEMENT }\end{array}$ & & & $\begin{array}{l}\text { FICIENT } \\
\text { PACITY } \\
\end{array}$ & $\begin{array}{c}\text { W/0 HORIZONTAL } \\
\text { EXII(s) }\end{array}$ & LORIZONTA & L EXIT/s) & OtRECT EXIT(s) \\
\hline ROUTES & -8 & & -2 & (0) & 3 & & 5 \\
\hline & NO MANUA & AL FIRE AL & ARM & MANUAL & FIRE ALARM & & \\
\hline 11. MANUAL FIRE & & & & W/O F.O. CONN. & 1. W/F.0.C & ONN. & \\
\hline & & -4 & & 1 & & & \\
\hline 12. SMOKE DETECTION & NONE & CORR & 100R ONLY & ROOMS ONLY & $\begin{array}{l}\text { CORRIDC } \\
\text { HABIT. S }\end{array}$ & $\begin{array}{l}\text { OR \& } \\
\text { PACE }\end{array}$ & TDTAL SPACE \\
\hline \& ALARM & 0 & & 2) & 3 & 4 & & 5 \\
\hline 13. AUTOMATIC & NONE & & RRIOOR & $\begin{array}{l}\text { CORRIOOR \& } \\
\text { HABIT. SPACE }\end{array}$ & TOTAL S & PACE & \\
\hline SPRINKLERS & 0 & & $2(0)^{\bullet \bullet}$ & 8 & & & \\
\hline
\end{tabular}

Figure 10. Life safety code requirements - new, sprinklered, one story facility 


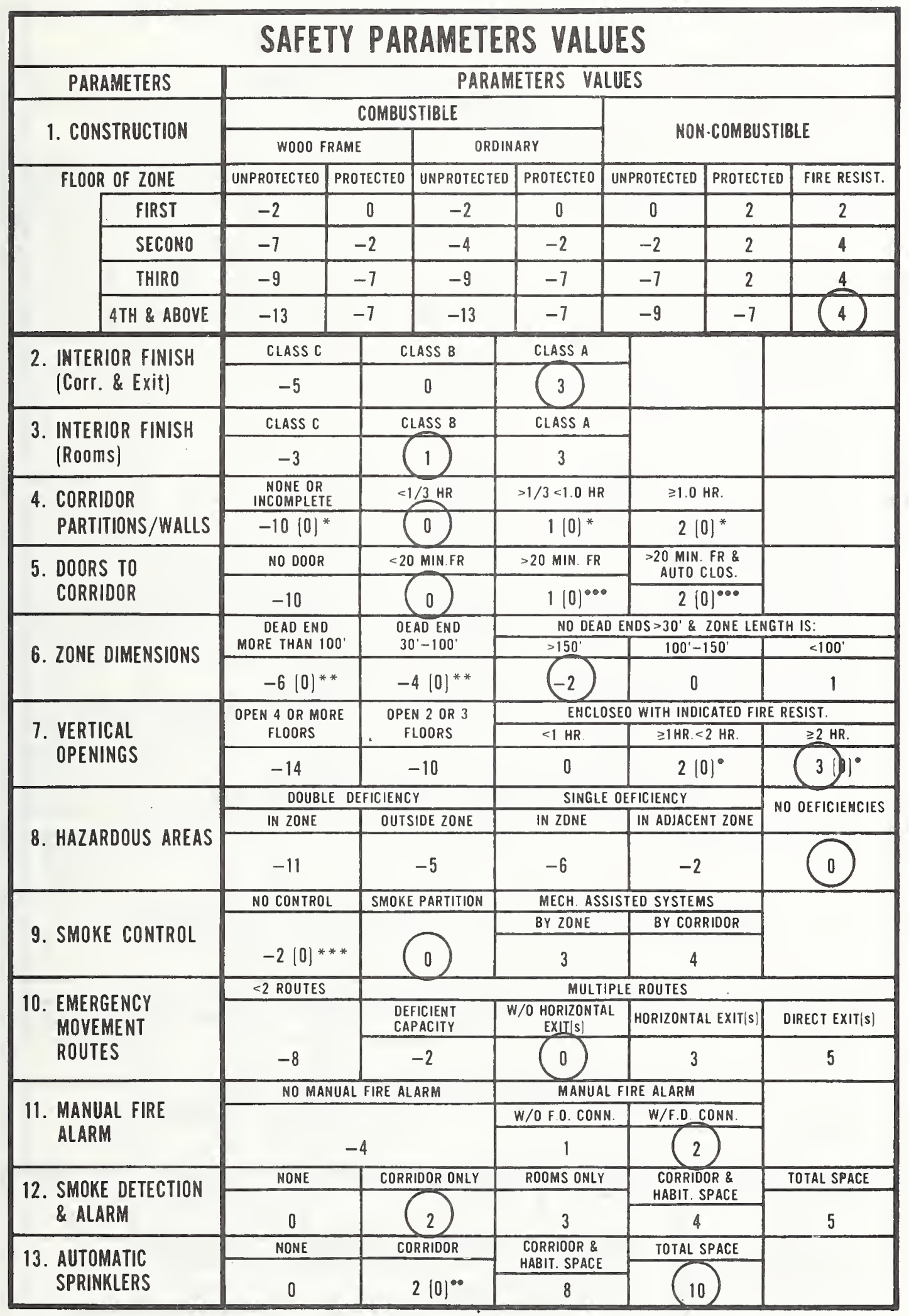

Figure 11. Life safety code requirements - new, sprinklered, more than three story facility 


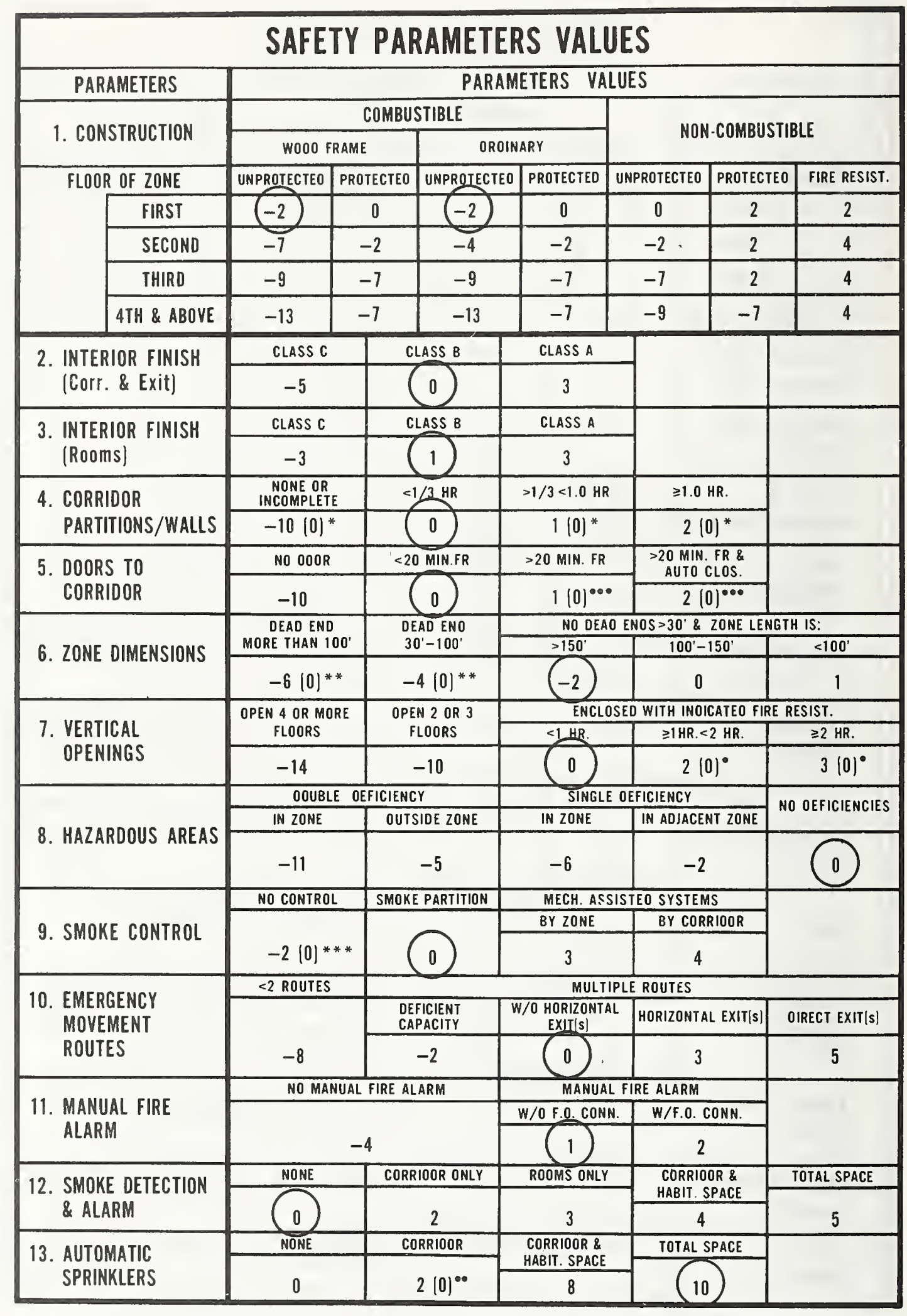

Figure 12. Life safety code requirements - existing, sprinklered one story facility 


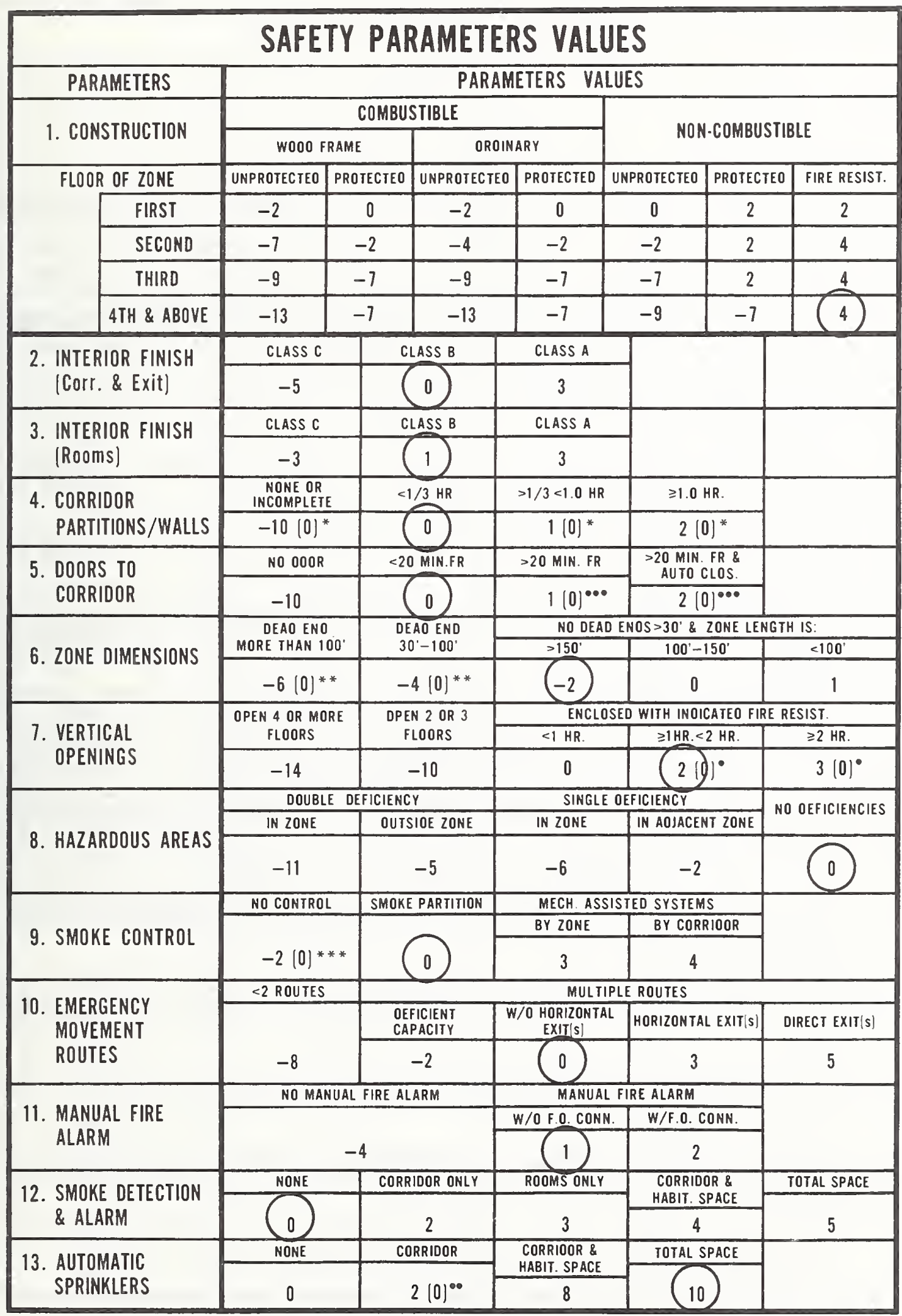

Figure 13. Life safety code requirements - existing, sprinklered more than three story facility 


\begin{tabular}{|c|c|c|c|c|}
\hline $\begin{array}{c}\text { SAFETY } \\
\text { PARAMETERS }\end{array}$ & NEW - 2 STORY & NEW - 3 STORY & $\begin{array}{l}\text { EXISTING - } \\
2 \text { STORY }\end{array}$ & $\begin{array}{l}\text { EXISTING - } \\
3 \text { STORY }\end{array}$ \\
\hline \multirow{2}{*}{ 1. CONSTRUCTION } & PROT. NON COMB. & PROT. NON COMB. & PROT. COMB. & PROT. NON COMB. \\
\hline & 2 & 2 & -2 & 2 \\
\hline \multirow{2}{*}{$\begin{array}{l}\text { 2. INTERIOR FINISH } \\
\text { (Corr. \& Exit) }\end{array}$} & CLASS A & CLASS A & CLASS B & CLASS B \\
\hline & 3 & 3 & 0 & 0 \\
\hline \multirow{2}{*}{$\begin{array}{l}\text { 3. INTERIOR FINISH } \\
\text { (Rooms) }\end{array}$} & CLASS B & CLASS B & CLASS B & CLASS B \\
\hline & 1 & 1 & 1 & 1 \\
\hline \multirow{2}{*}{$\begin{array}{l}\text { 4. CORRIDOR } \\
\text { PARTITIONS/WALLS }\end{array}$} & $<1 / 3 \mathrm{HR}$ & $<1 / 3 \mathrm{HR}$ & $<1 / 3 \mathrm{HR}$ & $<1 / 3 \mathrm{HR}$ \\
\hline & 0 & 0 & 0 & 0 \\
\hline \multirow{2}{*}{$\begin{array}{l}\text { 5. DOORS TO } \\
\text { CORRIDOR }\end{array}$} & $<20$ MIN & $<20$ MIN. & $<20$ MIN. & $<20$ MIN. \\
\hline & 0 & 0 & 0 & 0 \\
\hline \multirow{2}{*}{ 6. ZONE DIMENSIONS } & $>150^{\prime}$ & $>150^{\prime}$ & $>150^{\prime}$ & $>150^{\prime}$ \\
\hline & -2 & -2 & -2 & -2 \\
\hline \multirow{2}{*}{ 7. VERTICAL OPENINGS } & $\geqslant 1-<2 \mathrm{HR}$ & $\geqslant 1-<2 \mathrm{HR}$ & $\geqslant 1-<2 \mathrm{HR}$ & $\geqslant 1-<2 \mathrm{HR}$ \\
\hline & 2 & 2 & 2 & 2 \\
\hline \multirow{2}{*}{ 8. HAZARDOUS AREAS } & NO DEFICIENCIES & NO DEFICIENCIES & NO DEFICIENCIES & NO DEFICIENCIES \\
\hline & 0 & 0 & 0 & 0 \\
\hline \multirow{2}{*}{ 9. SMOKE CONTROL } & SMOKE PART. & SMOKE PART. & SMOKE PART. & SMOKE PART. \\
\hline & 0 & 0 & 0 & 0 \\
\hline \multirow{2}{*}{$\begin{array}{l}\text { 10. EMERGENCY } \\
\text { MOVEMENT ROUTES }\end{array}$} & MULTIPLE ROUTES & MULTIPLE ROUTES & MULTIPLE ROUTES & MULTIPLE ROUTES \\
\hline & 0 & 0 & 0 & 0 \\
\hline \multirow{2}{*}{$\begin{array}{l}\text { 11. MANUAL FIRE } \\
\text { ALARM }\end{array}$} & W/FD CONN. & W/FD CONN. & W/O/FD CONN. & W O FD CONN. \\
\hline & 2 & 2 & 1 & 1 \\
\hline \multirow{2}{*}{$\begin{array}{l}\text { 12. SMOKE DETECTION } \\
\text { \& ALARM }\end{array}$} & CORRIDOR ONLY & CORRIDOR ONLY & NONE & NONE \\
\hline & 2 & 2 & 0 & 0 \\
\hline \multirow{2}{*}{$\begin{array}{l}\text { 13. AUTOMATIC } \\
\text { SPRINKLERS }\end{array}$} & TOTAL SPACE & TOTAL SPACE & TOTAL SPACE & TOTAL SPACE \\
\hline & 10 & 10 & 10 & 10 \\
\hline TOTAL VALUE & 20 & 20 & 10 & 14 \\
\hline
\end{tabular}

Figure 14. Life safety code requirements - two and three stories (sprinklered) 


\begin{tabular}{|c|c|c|c|c|c|c|c|c|}
\hline PARAMETERS & \multicolumn{8}{|c|}{ PARAMETERS VALUES } \\
\hline \multirow{2}{*}{ 1. CONSTRUCTION } & \multicolumn{4}{|c|}{ COMBUSTIBLE } & \multirow{2}{*}{\multicolumn{4}{|c|}{ NON-COMBUSTIBLE }} \\
\hline & \multicolumn{2}{|c|}{ WOOO FRAME } & \multicolumn{2}{|c|}{ OROINARY } & & & & \\
\hline FLOOR OF ZONE & \begin{tabular}{|l|l} 
UNPROTECTEO & PR \\
\end{tabular} & PROTECTED & UNPROTECTED & D PROTECTED & UNPROTECTEO & \multicolumn{2}{|c|}{ PROTECTED } & FIRE RESIST. \\
\hline FIRST & -2 & 0 & -2 & 0 & 0 & 2 & & 2 \\
\hline SECOND & -7 & -2 & -4 & -2 & -2 & 2 & & 4 \\
\hline THIRD & -9 & -7 & -9 & -7 & -7 & 2 & & 4 \\
\hline 4TH \& ABOVE & -13 & -7 & -13 & -7 & -9 & -7 & & 4 \\
\hline \multirow{2}{*}{$\begin{array}{l}\text { 2. INTERIOR FINISH } \\
\text { (Corr. \& Exit) }\end{array}$} & CLASS C & & ASS B & CLASS A & & & & \\
\hline & -5 & & 0 & 3) & & & & \\
\hline \multirow{2}{*}{$\begin{array}{l}\text { 3. INTERIOR FINISH } \\
\text { (Rooms) }\end{array}$} & CLASS C & \multicolumn{2}{|c|}{ CLASS B } & CLASS A & & & & \\
\hline & -3 & & & 3 & & & & \\
\hline \multirow{2}{*}{$\begin{array}{l}\text { 4. CORRIDOR } \\
\text { PARTITIONS/WALLS } \\
\end{array}$} & $\begin{array}{c}\text { NONE OR } \\
\text { INCOMPLETE } \\
\end{array}$ & & & $>1 / 3<1.0 \mathrm{HR}$ & $\geqslant 1.0$ & & & \\
\hline & $-10(0)^{*}$ & & 0 & $1(0)^{*}$ & 21 & ) & & \\
\hline 5. DOORS TO & NO DOOR & & MIN.FR & $>20$ MIN. FR & $\begin{array}{l}>20 \text { MIN } \\
\text { AUTO }\end{array}$ & $\begin{array}{l}\text { FR \& } \\
\text { LOSS. }\end{array}$ & & \\
\hline CORRIDOR & -10 & & $0)$ & $1(0)^{\bullet \bullet}$ & 2 & je・ & & \\
\hline & DEAD END & & AD END & ND DEAD & ENDS > 30 \& & ZONE LEA & IGTH & \\
\hline 6. ZONE DIMENSIONS & MORE THAN 100' & & $-100^{\prime}$ & $>150^{\circ}$ & \begin{tabular}{l|l}
$100^{\circ}-$ \\
\end{tabular} & $150^{\prime}$ & & $<100^{\prime}$ \\
\hline & $-6(0)^{* *}$ & & $4(0)^{* *}$ & $(-2)$ & 0 & & & 1 \\
\hline & OPEN 4 OR MORE & $\mathrm{OPE}$ & $20 R 3$ & ENCLOS & SED WITH INDI & CATED FIR & E RE & SIST. \\
\hline 1. VERIILAL & FLOORS & & LORS & $<1 \mathrm{HR}$. & $\geqslant 1 \mathrm{HR} .<$ & $2 \mathrm{HR}$. & & $\geqslant 2 \mathrm{HR}$. \\
\hline UPENINGS & -14 & & -10 & 0 & 210 & & & $3(0)$ \\
\hline & DOUBLE D & DEFICIENC & & SINGLE 0 & OEFICIENCY & & & DEFICIENCIES \\
\hline 8 HA7ARONIIS ARFAS & IN ZONE & \begin{tabular}{|l|l|l|} 
& OUTS \\
\end{tabular} & 10E $20 N E$ & IN ZDNE & IN ADJACEI & NT ZONE & & \\
\hline 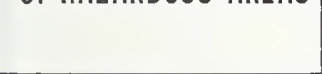 & -11 & & -5 & -6 & -2 & & & 0 \\
\hline & NO CONTROL & SMOKE & PARTITION & MECH. ASSIS & ISTED SYSTEM & & & \\
\hline 9. SMOKE CONTROL & & & & BY ZONE & \begin{tabular}{l|l} 
BY COR \\
\end{tabular} & RIDDR & & \\
\hline & $-2(0) * * *$ & & & 3 & 4 & & & \\
\hline & $<2$ ROUTES & & & MULTIP & PLE ROUTES & & & \\
\hline $\begin{array}{l}\text { TO. EMERGENLY } \\
\text { MOVEMENT }\end{array}$ & & & $\begin{array}{l}\text { FICIENT } \\
\text { PACITY }\end{array}$ & $\begin{array}{c}\text { W/O HORIZONTAL } \\
\text { EXIT(s) }\end{array}$ & HORIZDNTA & L EXIT/s) & & RECT EXIT/S\} \\
\hline ROUTES & -8 & & -2 & (0) & 3 & & & 5 \\
\hline & NO MANUAL & AL FIRE AL & ARM & MANUAL & FIRE ALARM & & & \\
\hline 11. MANUAL FIRE & & & & W/O F.D. CONN. & I. W/F.D. & ONN. & & \\
\hline & & -4 & & 1 & & & & \\
\hline 12. SMOKE DETECTION & NONE & CORR & IDOR ONLY & ROOMS ONLY & CDRRID & $O R \&$ & & OTAL SPACE \\
\hline \& ALARM & 0 & & & 3 & חNDil. & & & 5 \\
\hline 13. AUTOMATIC & NONE & & RRIOOR & $\begin{array}{l}\text { CORRIDOR \& } \\
\text { HABIT. SPACE }\end{array}$ & TOTALS & PACE & & \\
\hline SPRINKLERS & 0 & & $2(0)^{\bullet \bullet}$ & 8 & & & & \\
\hline
\end{tabular}

Figure 15. Life safety code requirements - new, sprinklered two story facility 


\begin{tabular}{|c|c|c|c|c|c|c|c|}
\hline \multicolumn{8}{|c|}{$\begin{array}{r}\text { SAFETY PARAMETERS VALUES } \\
\text { PARAMETERS VALUES }\end{array}$} \\
\hline \multirow{2}{*}{ 1. CONSTRUCTION } & \multicolumn{4}{|c|}{ COMBUSTIBLE } & \multirow{2}{*}{\multicolumn{3}{|c|}{ NON-COMBUSTIBLE }} \\
\hline & \multicolumn{2}{|c|}{ WOOO FRAME } & \multicolumn{2}{|c|}{ OROINARY } & & & \\
\hline FLOOR OF ZONE & \begin{tabular}{|l|l|l} 
UNPROTECTEO & PH \\
\end{tabular} & PROTECTED & UNPROTECTEO & PROTECTED & \multirow{2}{*}{$\begin{array}{c}\text { UNPROTECTED } \\
0 \\
\end{array}$} & \multirow{2}{*}{\begin{tabular}{|c|} 
PROTECTEO \\
2 \\
\end{tabular}} & \multirow{2}{*}{\begin{tabular}{|c|} 
FIRE RESIST. \\
2 \\
\end{tabular}} \\
\hline FIRST & -2 & 0 & -2 & 0 & & & \\
\hline SECOND & -7 & -2 & -4 & -2 & -2 & 2 & 4 \\
\hline THIRO & -9 & -7 & -9 & -7 & -7 & 2 & 4 \\
\hline 4TH \& ABOVE & -13 & -7 & -13 & -7 & -9 & -7 & 4 \\
\hline \multirow{2}{*}{$\begin{array}{l}\text { 2. INTERIOR FINISH } \\
\text { (Corr. \& Exit) }\end{array}$} & CLASS C & \multicolumn{2}{|c|}{ CLASS B } & CLASS A & & & \\
\hline & -5 & & 0 & (3) & & & \\
\hline \multirow{2}{*}{$\begin{array}{l}\text { 3. INTERIOR FINISH } \\
\text { (RoOms) }\end{array}$} & CLASS C & & ASS B & CLASS A & & & \\
\hline & -3 & & & 3 & & & \\
\hline \multirow{2}{*}{$\begin{array}{l}\text { 4. CORRIDOR } \\
\text { PARTITIONS/WALLS }\end{array}$} & $\begin{array}{c}\text { NONE OR } \\
\text { INCOMPLETE } \\
\end{array}$ & \multicolumn{2}{|c|}{$<1 / 3 \mathrm{HR}$} & $>1 / 3<1.0 \mathrm{HR}$ & \multicolumn{2}{|c|}{$\geqslant 1.0 \mathrm{HR}$. } & \\
\hline & $-10(0)^{*}$ & & 0) & $1(0)^{*}$ & \multicolumn{2}{|c|}{$2(0)^{*}$} & \\
\hline \multirow{2}{*}{$\begin{array}{l}\text { 5. DOORS TO } \\
\text { CORRIDOR } \\
\end{array}$} & NO OOOR & & MIN.FR & $>20$ MIN. FR & $\begin{array}{l}>20 \mathrm{MIN} \\
\text { AUTO }\end{array}$ & $\begin{array}{l}\text { FR \& } \\
\text { CLOS. }\end{array}$ & \\
\hline & -10 & & $0)$ & $1|0|^{\cdots \infty}$ & & $0]^{\bullet \cdots}$ & \\
\hline & OEAD END & & DD END & ND DEAD & ENDS $>30^{\prime} \&$ & ZONE LEN & GIH IS: \\
\hline 6. ZONE DIMENSIONS & MORE THAN 100 & & $'-100$ & $>150^{\circ}$ & \begin{tabular}{l|l}
$100^{\prime}-$ \\
\end{tabular} & & $<100^{\circ}$ \\
\hline & $-6(0)^{* *}$ & & $1(0)^{* *}$ & $(-2)$ & 0 & & 1 \\
\hline & OPEN 4 OR MORE & OPEI & $20 R 3$ & ENCLOS & SED WITH INOI & CATEO FIR & E RESIST. \\
\hline 7. VERTICAL & FLOORS & & OORS & $<1 \mathrm{HR}$. & \begin{tabular}{l|l}
$1 \mathrm{HR}$. \\
\end{tabular} & $2 \mathrm{HR}$. & $\geqslant 2 \mathrm{HR}$ \\
\hline & -14 & & -10 & 0 & & ]$^{\bullet}$ & $3|0|^{\bullet}$ \\
\hline & OOUBLE & OEFICIENC & & SINGLE & OEFICIENCY & & NO OEF ICIENCIES \\
\hline 8. HAZARDOUS AREAS & IN ZONE & \begin{tabular}{l|l} 
OUTS \\
\end{tabular} & IDE ZONE & IN ZONE & \begin{tabular}{|l|l} 
IN AOIACE \\
\end{tabular} & IT ZONE & \\
\hline & -11 & & -5 & -6 & -2 & & \\
\hline & NO CONTROL & SMOKE & PARIITION & MECH. ASSI & ISTED SYSTER & & \\
\hline 9. SMOKE CONTROL & & & & BY ZONE & BY COR & RIDOR & \\
\hline & $-2|0| * * *$ & & & 3 & 4 & & \\
\hline & $\angle 2$ ROUTES & & & MULTIP & PLE ROUTES & & \\
\hline $\begin{array}{l}\text { 10. EMLRLEACT } \\
\text { MOVEMENT }\end{array}$ & & & $\begin{array}{l}\text { IIIENT } \\
\text { ACITY } \\
\end{array}$ & $\begin{array}{c}\begin{array}{c}\text { W/0 HORIZONTAL } \\
\text { EXIIIs! }\end{array} \\
\end{array}$ & HORIZONTA & L EXIT/S| & DIRECT EXIT(S) \\
\hline ROUTES & -8 & & -2 & (0) & 3 & & 5 \\
\hline & NO MANU & JAL FIRE ALI & & MANUAL & FIRE ALARM & & \\
\hline 11. MANUAL FIRE & & & & W/O F.O. CONN & W/F.0.0 & & \\
\hline & & -4 & & 1 & & & \\
\hline 12. SMOKE DETECTION & NONE & \begin{tabular}{l|l} 
CORRI \\
\end{tabular} & DOR ONLY & ROOMS ONLY & $\begin{array}{l}\text { CORRID } \\
\text { HABITS S }\end{array}$ & OR \& & TOTAL SPACE \\
\hline \& ALARM & 0 & & 2) & 3 & 4 & & 5 \\
\hline 13. AUTOMATIC & NONE & & RRIDOR & $\begin{array}{l}\text { CORRIOOR \& } \\
\text { HABIT. SPACE }\end{array}$ & TOTAL S & PACE & \\
\hline SPRINKLERS & 0 & & $(0)^{\bullet \bullet}$ & 8 & & & \\
\hline
\end{tabular}

Figure 16. Life safety code requirements - new, sprinklered three story facility 


\begin{tabular}{|c|c|c|c|c|c|c|c|}
\hline \multicolumn{8}{|c|}{ SAFETY PARAMETERS VALUES } \\
\hline \multirow{2}{*}{ 1. CONSTRUCTION } & \multicolumn{4}{|c|}{\begin{tabular}{|c|} 
COMBUSTIBLE \\
\end{tabular}} & \multirow{2}{*}{\multicolumn{3}{|c|}{ NON-COMBUSTIBLE }} \\
\hline & \multicolumn{2}{|c|}{ WOOD FRAME } & \multicolumn{2}{|c|}{ ORDINARY } & & & \\
\hline FLOOR OF ZONE & \begin{tabular}{|l|l|} 
UNPROTECTED & P \\
\end{tabular} & \multirow{2}{*}{\begin{tabular}{|c|} 
PROTECTEO \\
0 \\
\end{tabular}} & \multirow{2}{*}{$\begin{array}{c}\text { UNPROTECTEO } \\
-2 \\
\end{array}$} & \multirow{2}{*}{\begin{tabular}{|c|} 
PROTECTEO \\
8 \\
\end{tabular}} & \multirow{2}{*}{\begin{tabular}{|c|} 
UNPROTECTED \\
0 \\
\end{tabular}} & \multirow{2}{*}{\begin{tabular}{|c|} 
PROTECTEO \\
2 \\
\end{tabular}} & \multirow{2}{*}{\begin{tabular}{|c|} 
FIRE RESIST. \\
2 \\
\end{tabular}} \\
\hline FIRST & -2 & & & & & & \\
\hline SECOND & -7 & $(-2$ & -4 & $(-2)$ & $(-2)$ & 2 & 4 \\
\hline THIRD & -9 & -7 & -9 & -7 & -7 & 2 & 4 \\
\hline 4TH \& ABOVE & -13 & -7 & -13 & -7 & -9 & -7 & 4 \\
\hline \multirow{2}{*}{$\begin{array}{l}\text { 2. INTERIOR FINISH } \\
\text { (Corr. \& Exit) }\end{array}$} & \multicolumn{3}{|l|}{ CLASS C } & CLASS A & & & \\
\hline & -5 & & $0)$ & 3 & & & \\
\hline \multirow{2}{*}{$\begin{array}{l}\text { 3. INTERIOR FINISH } \\
\text { (Rooms) }\end{array}$} & \multicolumn{3}{|c|}{ CLASS B } & CLASS A & & & \\
\hline & -3 & & 1) & 3 & & & \\
\hline \multirow{2}{*}{$\begin{array}{l}\text { 4. CORRIDOR } \\
\text { PARTITIONS/WALLS }\end{array}$} & $\begin{array}{l}\text { NONE OR } \\
\text { INCOMPLETE }\end{array}$ & \multicolumn{2}{|c|}{$<1 / 3 \mathrm{HR}$} & $>1 / 3<1.0 \mathrm{HR}$ & \multicolumn{2}{|c|}{$\geqslant 1.0 \mathrm{HR}$. } & \\
\hline & $-10(0)^{*}$ & & $0)$ & $1(0)^{*}$ & 210 & & \\
\hline 5. DOORS TO & NO DOOR & $<20$ & MIN.FR & $>20$ MIN. FR & $\begin{array}{l}>20 \text { MIN. } \\
\text { AUTOC }\end{array}$ & $\begin{array}{l}\text { FR\& } \\
\text { FRS. } \\
\text { LOS. }\end{array}$ & \\
\hline CORRIDOR & -10 & & $0)$ & $1(0)^{\cdots}$ & 210 & 10 & \\
\hline & $\begin{array}{c}\text { DEAD END } \\
\text { MEA }\end{array}$ & & $\begin{array}{l}\text { DEND } \\
-100\end{array}$ & NO OEAD & ENOS $>30^{\prime} \&$ & ZONE LEN & ITH IS: \\
\hline 6. ZONE DIMENSIONS & & & & $>150^{\prime}$ & $100^{\prime}-1$ & & $\leq 100^{\prime}$ \\
\hline & $-6(0)^{* *}$ & & $(0)^{* *}$ & $(-2)$ & 0 & & 1 \\
\hline & OPEN 4 OR MORE & OPEN & $20 R 3$ & ENCLOS & SED WITH INOIC & CATEO FIR & RESIST. \\
\hline 7. VERTICAL & FLOORS & & OORS & $<1 \mathrm{HR}$. & $\geqslant 1 \mathrm{HR}<$ & $2 \mathrm{HR}$. & $\geqslant 2 \mathrm{HR}$. \\
\hline & -14 & & 10 & 0 & $(210$ & & $3|0|^{\circ}$ \\
\hline & DOUBLE & DEFICIENCY & & SINGLE D & DEFICIENCY & & \\
\hline 8 HAZAPAOUS ADEAS & IN ZONE & \begin{tabular}{l|l} 
& OUTS \\
\end{tabular} & DE ZONE & IN ZONE & IN ADJACEN & IT ZONE & NO DEFICIENCIES \\
\hline 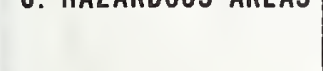 & -11 & & -5 & -6 & -2 & & \\
\hline & NO CONTROL & SMOKE & PARTITION & MECH. ASSIS & ISTED SYSTEM & & \\
\hline 9. SMOKE CONTROL & & & & BY ZONE & \begin{tabular}{l|l} 
& $B Y C O R R$ \\
\end{tabular} & IDOR & \\
\hline & $-2(0)^{* * *}$ & & & 3 & 4 & & \\
\hline & $<2$ ROUTES & & & MULTIPI & PLE ROUTES & & \\
\hline $\begin{array}{l}\text { 10. EMLRLiERLY } \\
\text { MOVEMENT }\end{array}$ & & & $\begin{array}{l}\text { CIENT } \\
\text { ACITY } \\
\end{array}$ & $\begin{array}{l}\text { W/O HORIZONTAL } \\
\text { EXIT(s) }\end{array}$ & HORIZONTAL & LXXIT/s] & DIRECT EXIT/S| \\
\hline ROUTES & -8 & & -2 & $(0)$ & 3 & & 5 \\
\hline & NO MANU & UAL FIRE ALA & & MANUAL & FIRE ALARM & & \\
\hline 11. MANUAL FIRE & & & & W/OF.D CONN. & \begin{tabular}{l|l} 
W/F.0. C \\
\end{tabular} & ONN. & \\
\hline & & -4 & & (1) & 2 & & \\
\hline 12. SMOKE DETECTION & NONE & CORRI & SOR ONLY & ROOMS ONLY & CORRIDC & $\begin{array}{l}P R \& \\
P A C E\end{array}$ & TOTAL SPACE \\
\hline \& ALARM & 0 & & 2 & 3 & 4 & & 5 \\
\hline 13. AUTOMATIC & NONE & & RIDOR & $\begin{array}{l}\text { CORRIOOR \& } \\
\text { HABIT. SPACE }\end{array}$ & TOTALS & PACE & \\
\hline SPRINKLERS & 0 & & $(0)^{\circ \bullet}$ & 8 & & & \\
\hline
\end{tabular}

Figure 17. Life safety code requirements - existing, sprinklered two story facility 


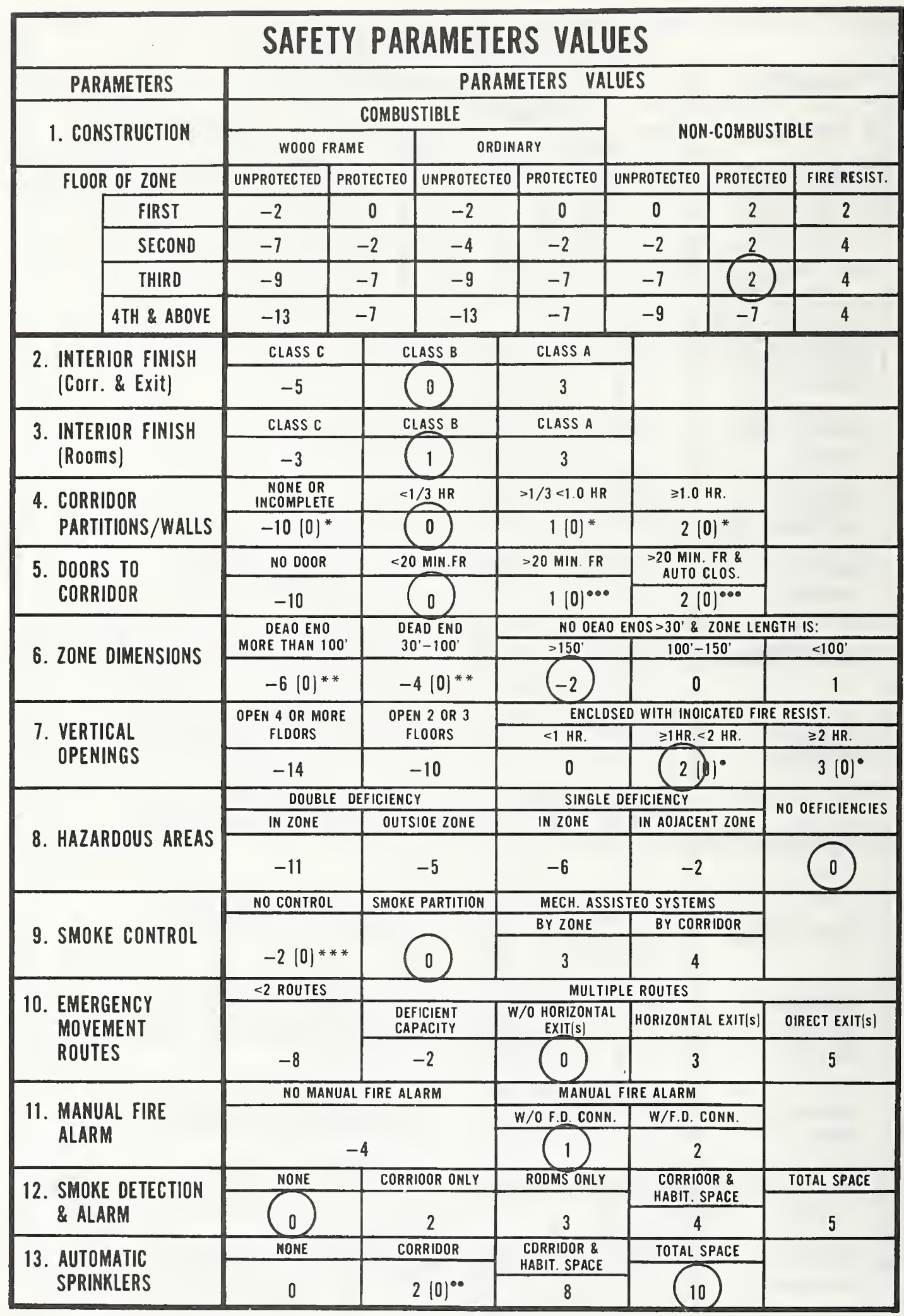

Figure 18. Life safety code requirements - existing, sprinklered three story facility 


\section{INDIVIDUAL SAFETY EVALUATIONS}

\begin{tabular}{|c|c|c|c|c|}
\hline $\begin{array}{c}\text { SAFETY } \\
\text { PARAMETERS }\end{array}$ & $\begin{array}{l}\text { CONTAINMENT } \\
\text { SAFETY }\end{array}$ & $\begin{array}{c}\text { EXTINGUISHMENT } \\
\text { SAFETY }\end{array}$ & $\begin{array}{l}\text { PEOPLE } \\
\text { MOVEMENT } \\
\text { SAFETY }\end{array}$ & $\begin{array}{l}\text { GENERAL } \\
\text { SAFETY }\end{array}$ \\
\hline \multicolumn{5}{|l|}{ 1. CONSTRUCTION } \\
\hline \multicolumn{5}{|l|}{$\begin{array}{l}\text { 2. INTERIOR FINISH } \\
\text { (Corr. \& Exit) }\end{array}$} \\
\hline \multicolumn{5}{|l|}{$\begin{array}{l}\text { 3. INTERIOR FINISH } \\
\text { (ROOMS) }\end{array}$} \\
\hline \multicolumn{5}{|l|}{$\begin{array}{l}\text { 4. CORRIDOR } \\
\text { PARTITIONS/WALLS }\end{array}$} \\
\hline \multicolumn{5}{|l|}{$\begin{array}{l}\text { 5. DOORS TO } \\
\text { CORRIDOR }\end{array}$} \\
\hline \multicolumn{5}{|l|}{ 6. ZONE DIMENSIONS } \\
\hline \multicolumn{5}{|l|}{ 7. VERTICAL OPENINGS } \\
\hline \multicolumn{5}{|l|}{ 8. HAZARDOUS AREAS } \\
\hline \multicolumn{5}{|l|}{ 9. SMOKE CONTROL } \\
\hline \multicolumn{5}{|l|}{$\begin{array}{l}\text { 10. EMERGENCY } \\
\text { MOVEMENT ROUTES }\end{array}$} \\
\hline \multicolumn{5}{|l|}{$\begin{array}{l}\text { 11. MANUAL FIRE } \\
\text { ALARM }\end{array}$} \\
\hline \multicolumn{5}{|l|}{$\begin{array}{l}\text { 12. SMOKE DETECTION } \\
\text { \& ALARM }\end{array}$} \\
\hline \multicolumn{5}{|l|}{$\begin{array}{l}\text { 13. AUTOMATIC } \\
\text { SPRINKLERS }\end{array}$} \\
\hline & & & & \\
\hline
\end{tabular}

Figure 19. Individual safety evaluations 


\begin{tabular}{|c|c|c|c|c|}
\hline $\begin{array}{c}\text { SAFETY } \\
\text { PARAMETERS }\end{array}$ & NEW - I STORY & $\begin{array}{l}\text { NEW - MORE } \\
\text { THAN I STORY }\end{array}$ & $\begin{array}{l}\text { EXISTING } \\
1 \text { STORY }\end{array}$ & $\begin{array}{l}\text { EXISTING - } \\
\text { MORE THAN } \\
1 \text { STORY }\end{array}$ \\
\hline \multirow{2}{*}{ 1. CONSTRUCTION } & PROT. NON COMB. & FIRE RESISTIVE & PROT. NON COMB. & FIRE RESISTIVE \\
\hline & 2 & 4 & 2 & 4 \\
\hline \multirow{2}{*}{$\begin{array}{l}\text { 2. INTERIOR FINISH } \\
\text { (Corr. \& Exit) }\end{array}$} & CLASS A & CLASS A & CLASS B & CLASS B \\
\hline & 3 & 3 & 0 & 0 \\
\hline \multirow{2}{*}{$\begin{array}{l}\text { 3. INTERIOR FINISH } \\
\text { (Rooms] }\end{array}$} & CLASS B & CLASS B & CLASS B & CLASS B \\
\hline & 1 & 1 & 1 & 1 \\
\hline \multirow{2}{*}{$\begin{array}{l}\text { 4. CORRIDOR } \\
\text { PARTITIONS/WALLS }\end{array}$} & $\geqslant 1.0 \mathrm{HR}$ & $\geqslant 1.0 \mathrm{HR}$ & $<1 / 3 \mathrm{HR}$ & $<1 / 3 H R$ \\
\hline & 2 & 2 & 0 & 0 \\
\hline \multirow{2}{*}{$\begin{array}{l}\text { 5. DOORS TO } \\
\text { CORRIDOR }\end{array}$} & $\geq 20 \mathrm{MIN}$. & $\geq 20$ MIN. & $\geq 20$ MIN. & $\geqslant 20 \mathrm{MIN}$ \\
\hline & 1 & 1 & 1 & 1 \\
\hline \multicolumn{5}{|l|}{ 6. ZONE DIMENSIONS } \\
\hline \multirow{2}{*}{ 7. VERTICAL OPENINGS } & NON APP. & $2 \mathrm{HR}$ & NON APP. & $\geqslant 1-<2 \mathrm{HR}$ \\
\hline & $\begin{array}{l}0 \\
\end{array}$ & 3 & 0 & 2 \\
\hline \multirow{2}{*}{ 8. HAZARDOUS AREAS } & NO DEFICIENCIES & NO DEFICIENCIES & NO DEFICIENCIES & NO DEFICIENCIES \\
\hline & 0 & 0 & 0 & 0 \\
\hline \multicolumn{5}{|l|}{ 9. SMOKE CONTROL } \\
\hline \multicolumn{5}{|l|}{$\begin{array}{l}\text { 10. EMERGENCY } \\
\text { MOVEMENT ROUTES }\end{array}$} \\
\hline \multicolumn{5}{|l|}{$\begin{array}{l}\text { 11. MANUAL FIRE } \\
\text { ALARM }\end{array}$} \\
\hline \multicolumn{5}{|l|}{$\begin{array}{l}\text { 12. SMOKE DETECTION } \\
\text { \& ALARM }\end{array}$} \\
\hline \multirow{2}{*}{$\begin{array}{l}\text { 13. AUTOMATIC } \\
\text { SPRINKLERS }\end{array}$} & NONE & NONE & NONE & NONE \\
\hline & 0 & 0 & 0 & 0 \\
\hline TOTAL VALUE & 9 & 14 & 4 & 8 \\
\hline
\end{tabular}

Figure 20. Minimum containment safety (non-sprinklered) 


\begin{tabular}{|c|c|c|c|c|}
\hline $\begin{array}{c}\text { SAFETY } \\
\text { PARAMETERS }\end{array}$ & NEW - 1 STORY & $\begin{array}{l}\text { NEW - MORE } \\
\text { THAN } 1 \text { STORY }\end{array}$ & $\begin{array}{l}\text { EXISTING - } \\
1 \text { STORY }\end{array}$ & $\begin{array}{l}\text { EXISTING - } \\
\text { MORE THAN } \\
1 \text { STORY }\end{array}$ \\
\hline \multirow{2}{*}{ 1. CONSTRUCTION } & PROT. NON COMB. & FIRE RESISTIVE & PROT. NON COMB. & FIRE RESISTIVE \\
\hline & 2 & 4 & 2 & 4 \\
\hline \multicolumn{5}{|l|}{$\begin{array}{l}\text { 2. INTERIOR FINISH } \\
\text { (Corr. \& Exit) }\end{array}$} \\
\hline \multicolumn{5}{|l|}{$\begin{array}{l}\text { 3. INTERIOR FINISH } \\
\text { (Rooms) }\end{array}$} \\
\hline \multicolumn{5}{|l|}{$\begin{array}{l}\text { 4. CORRIDOR } \\
\text { PARTITIONS/WALLS }\end{array}$} \\
\hline \multicolumn{5}{|l|}{$\begin{array}{l}\text { 5. DOORS TO } \\
\text { CORRIDOR }\end{array}$} \\
\hline \multicolumn{5}{|l|}{ 6. ZONE DIMENSIONS } \\
\hline \multicolumn{5}{|l|}{ 7. VERTICAL OPENINGS } \\
\hline 8. HAZARDOUS AREAS & $\begin{array}{c}\text { NO DEFICIENCIES } \\
0\end{array}$ & $\begin{array}{c}\text { NO DEFICIENCIES } \\
0\end{array}$ & $\begin{array}{c}\text { NO DEFICIENCIES } \\
0\end{array}$ & $\begin{array}{c}\text { NO DEFICIENCIES } \\
0\end{array}$ \\
\hline \multicolumn{5}{|l|}{ 9. SMOKE CONTROL } \\
\hline \multicolumn{5}{|l|}{$\begin{array}{l}\text { 10. EMERGENCY } \\
\text { MOVEMENT ROUTES }\end{array}$} \\
\hline \multirow{2}{*}{$\begin{array}{l}\text { 11. MANUAL FIRE } \\
\text { ALARM }\end{array}$} & W/FO CONN. & W/ FO CONN. & W/O/FO CONN. & W/O/FO CONN. \\
\hline & 2 & 2 & 1 & 1 \\
\hline \multirow{2}{*}{$\begin{array}{l}\text { 12. SMOKE DETECTION } \\
\text { \& ALARM }\end{array}$} & CORRIDOR ONLY & CORRIDOR ONLY & NONE & NONE \\
\hline & 2 & 2 & 0 & 0 \\
\hline \multirow{2}{*}{$\begin{array}{l}\text { 13. AUTOMATIC } \\
\text { SPRINKLERS }\end{array}$} & NONE & NONE & NONE & NONE \\
\hline & 0 & 0 & 0 & 0 \\
\hline TOTAL VALUE & 6 & 8 & 3 & 5 \\
\hline
\end{tabular}

Figure 21. Minimum extinguishment safety (non-sprinklered) 


\begin{tabular}{|c|c|c|c|c|}
\hline $\begin{array}{c}\text { SAFETY } \\
\text { PARAMETERS }\end{array}$ & NEW - I STORY & $\begin{array}{l}\text { NEW - MORE } \\
\text { THAN } 1 \text { STORY }\end{array}$ & $\begin{array}{l}\text { EXISTING - } \\
1 \text { STORY }\end{array}$ & $\begin{array}{l}\text { EXISTING - } \\
\text { MORE THAN } \\
1 \text { STORY }\end{array}$ \\
\hline \multicolumn{5}{|l|}{ 1. CONSTRUCTION } \\
\hline \multirow{2}{*}{$\begin{array}{l}\text { 2. INTERIOR FINISH } \\
\text { (Corr. \& Exit) }\end{array}$} & CLASS A & CLASS A & CLASS B & CLASS B \\
\hline & 3 & 3 & 0 & 0 \\
\hline \multicolumn{5}{|l|}{$\begin{array}{l}\text { 3. INTERIOR FINISH } \\
\text { (ROOMS) }\end{array}$} \\
\hline \multicolumn{5}{|l|}{$\begin{array}{l}\text { 4. CORRIDOR } \\
\text { PARTITIONS/WALLS }\end{array}$} \\
\hline \multirow{2}{*}{$\begin{array}{l}\text { 5. DOORS TO } \\
\text { CORRIDOR }\end{array}$} & $\geqslant 20 \mathrm{MIN}$. & $\geqslant 20$ MIN. & $\geqslant 20 \mathrm{MIN}$. & $\geqslant 20 \mathrm{MIN}$ \\
\hline & 1 & 1 & 1 & 1 \\
\hline \multirow{2}{*}{ 6. ZONE DIMENSIONS } & $100^{\prime}-150^{\prime}$ & $100^{\prime}-150^{\prime}$ & $100^{\prime}-150^{\prime}$ & $100^{\prime}-150^{\prime}$ \\
\hline & 0 & 0 & 0 & 0 \\
\hline \multirow{2}{*}{ 7. VERTICAL OPENINGS } & NON APP. & $2 \mathrm{HR}$ & NON APP. & $\geqslant 1-<2 H R$ \\
\hline & 0 & 3 & 0 & 2 \\
\hline \multicolumn{5}{|l|}{ 8. HAZARDOUS AREAS } \\
\hline \multirow{2}{*}{ 9. SMOKE CONTROL } & SMOKE PART & SMOKE PART. & SMOKE PART. & SMOKE PART \\
\hline & 0 & 0 & 0 & 0 \\
\hline \multirow{2}{*}{$\begin{array}{l}\text { 10. EMERGENCY } \\
\text { MOVEMENT ROUTES }\end{array}$} & MULTIPLE ROUTES & MULTIPLE ROUTES & MULTIPLE ROUTES & MULTIPLE ROUTES \\
\hline & 0 & 0 & 0 & 0 \\
\hline $\begin{array}{l}\text { 11. MANUAL FIRE } \\
\text { ALARM }\end{array}$ & & & & \\
\hline \multirow{2}{*}{$\begin{array}{l}\text { 12. SMOKE DETECTION } \\
\text { \& ALARM }\end{array}$} & CORRIDOR ONLY & CORRIDOR ONLY & NONE & NONE \\
\hline & 2 & 2 & 0 & 0 \\
\hline \multirow{2}{*}{$\begin{array}{l}\text { 13. AUTOMATIC } \\
\text { SPRINKLERS }\end{array}$} & NONE & NONE & NONE & NONE \\
\hline & 0 & 0 & 0 & 0 \\
\hline TOTAL VALUE & 6 & 9 & 1 & 3 \\
\hline
\end{tabular}

Figure 22. Minimum people movement safety (non-sprinklered) 
FIRE/SMOKE ZONE* EVALUATION WORK SHEET FOR HEALTH CARE FACILITIES

FACILITY BUILDING

ZONE(S) EVALUATED

EVALUATOR

DATE

Complete this work sheet for each zone. Where conditions are the same in several zones, one work sheet can be used for those zones.

Step 1: Determine Occupancy Risk Parameter Factors - Use Table I.

A. For each Risk Parameter in Table I, select and circle the appropriate risk factor value. Choose only one for each of the five Risk Parameters.

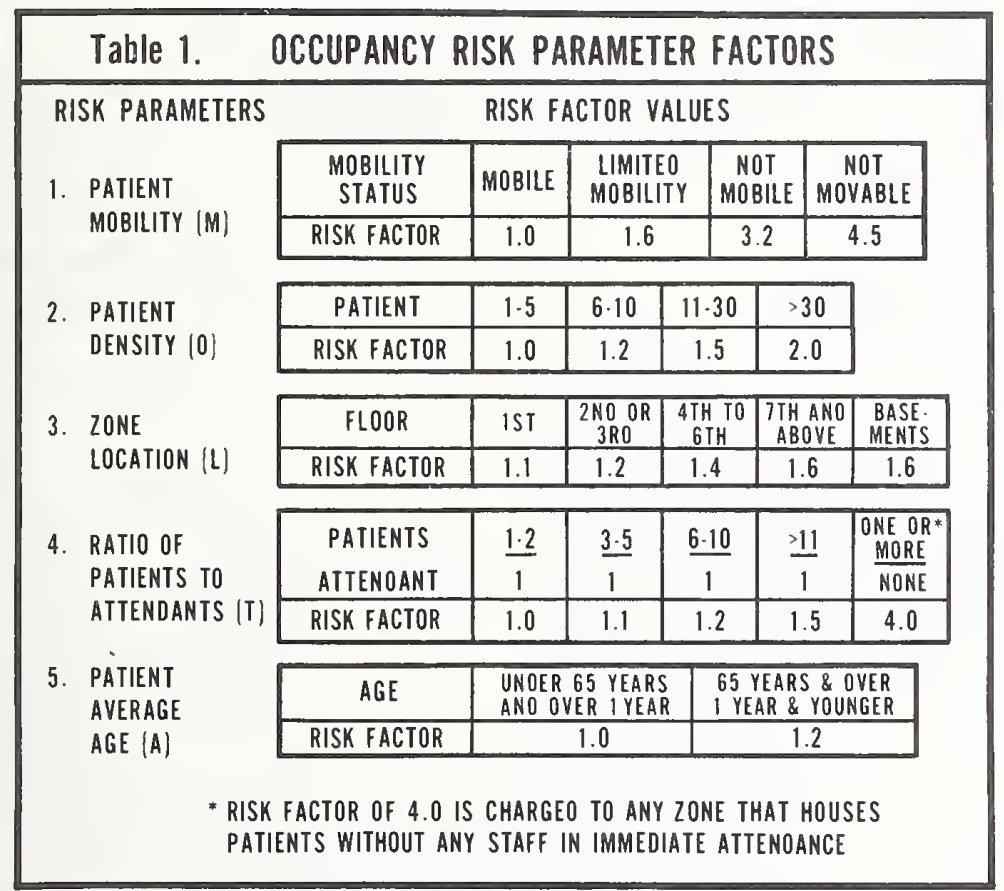

Step 2: Compute Occupancy Risk Factor (F) - Use Table 2.

A. Transfer the circled risk factor values from Table 1 to the corresponding blocks in Table 2.

B. Compute $\mathrm{F}$ by multiplying the risk factor values as indicated in Table 2 .

\section{Table 2. OCCUPANCY RISK FACTOR CALCULATION}

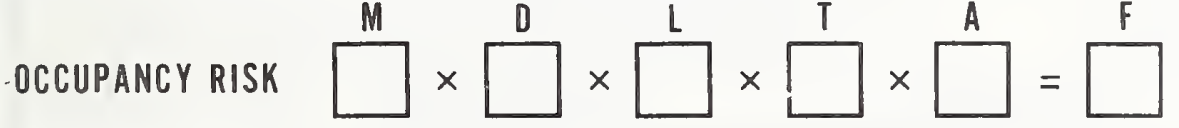

Step 3: Compute Adjusted Building Status (R) - Use Table 3A or 3B.

A. If building is classified as NEW use Table 3A. If building is classified as existing use Table $3 \mathrm{~B}$.

B. Transfer the value of $F$ from Table 2 to Table $3 \mathrm{~A}$ or Table $3 \mathrm{~B}$ as appropriate. Calculate "R."

C. Transfer " $R$ " to the block labeled " $R$ " in Table 7 on page 4 of the work sheet.
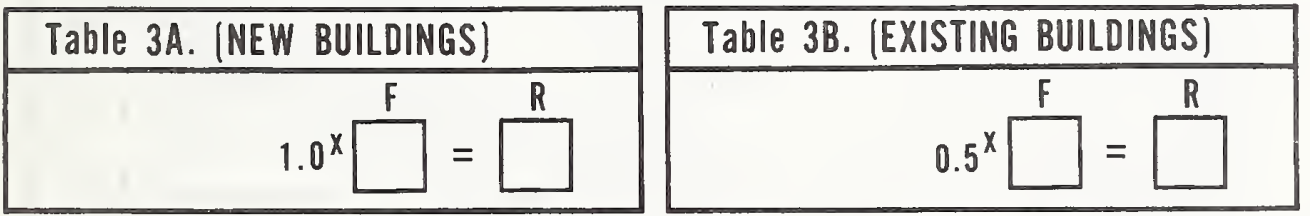

* FIRE/SMOKE ZONE is a space separated from all other spaces by floors, horizontal exits, or smoke barriers.

rigure 23a. Fire/smoke zone evaluation work sheet for health care facilities 
Step 4: Determine Safety Parameter Values - Use Table 4.

A. Select and circle the safety value for each safety parameter in Table 4 that best describes the conditions in the zone. Choose only one value for each of the 13 parameters. If two or more appear to apply choose the one with the lowest print value.

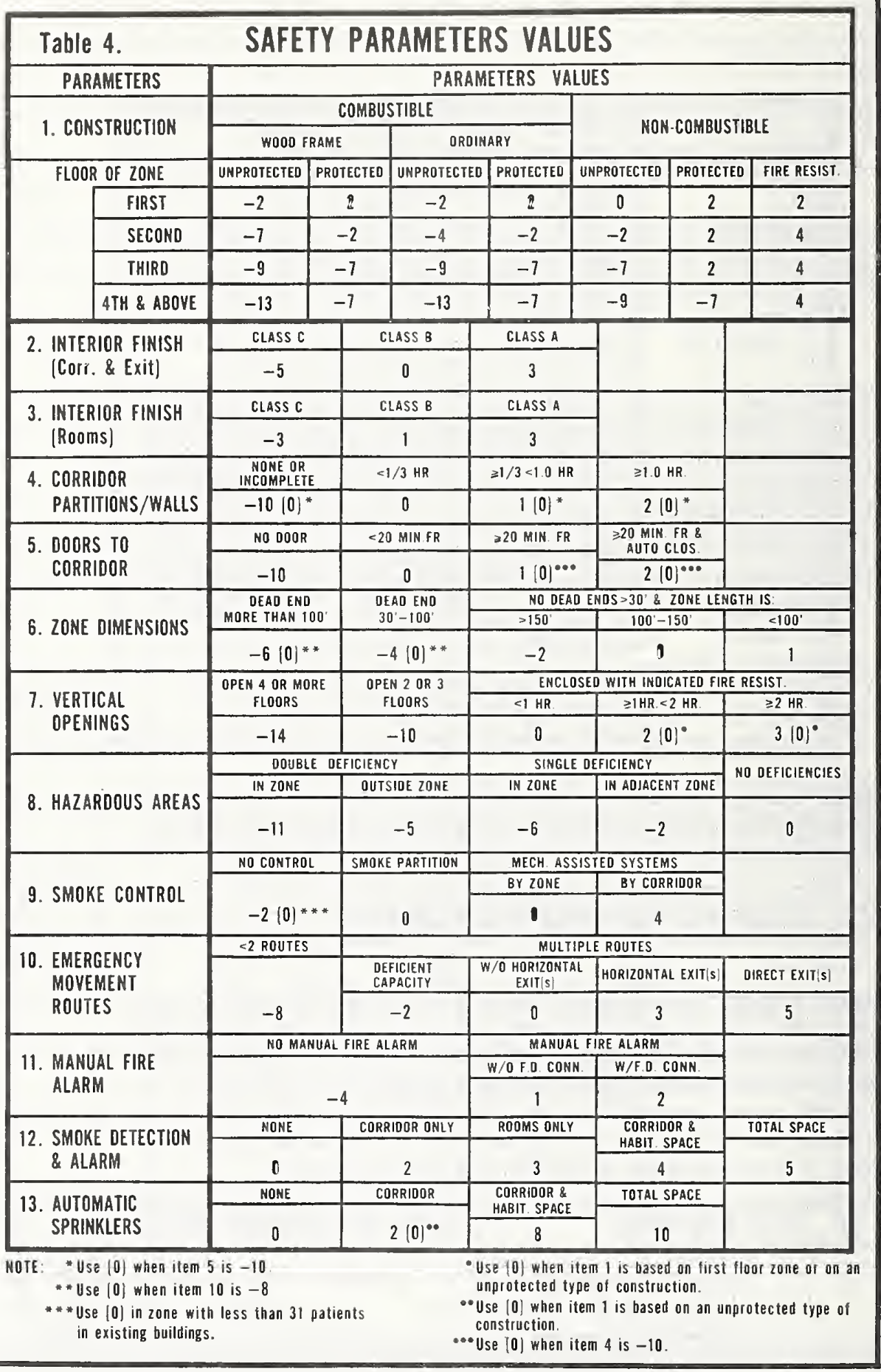

Figure 23b. Fire/smoke zone evaluation work sheet for health care facilities 
Step 5: Compute Individual Safety Evaluations - Use Table 5.

A. Transfer each of the 13 circled Safety Parameter Value from Table 4 to every unshaded blocks in the line with the corresponding Safety Parameter in Table 5. For Safety Parameter 13 (Sprinklers) the value entered in the (People Movement Safety) is recorded in Table 5 as $1 / 2$ the corresponding value circled in Table 4.

B. Add the four columns, keeping in mind that any negative numbers deduct.

C. Transfer the resulting total values for $\mathrm{S}_{1}, \mathrm{~S}_{2}, \mathrm{~S}_{3}, \mathrm{~S}_{\mathrm{G}}$ to the blocks labeled $\mathrm{S}_{1}, \mathrm{~S}_{2}, \mathrm{~S}_{3}, \mathrm{~S}_{\mathrm{G}}$ in Table 7 on page 4 of this sheet.

\section{Table 5. INDIVIDUAL SAFETY EVALUATIONS}

\begin{tabular}{|c|c|c|c|c|}
\hline $\begin{array}{c}\text { SAFETY } \\
\text { PARAMETERS }\end{array}$ & $\begin{array}{l}\text { CONTAINMENT } \\
\text { SAFETY } \\
{\left[S_{1} \mid\right.}\end{array}$ & $\begin{array}{c}\text { EXTINGUISHMENT } \\
\text { SAFETY } \\
\left|S_{2}\right|\end{array}$ & $\begin{array}{c}\text { PEOPLE } \\
\text { MOVEMENT } \\
\text { SAFETY }\left(S_{3}\right)\end{array}$ & $\begin{array}{c}\text { GENERAL } \\
\text { SAFETY } \\
\text { [SG] }\end{array}$ \\
\hline 1. CONSTRUCTION & & & & \\
\hline $\begin{array}{l}\text { 2. INTERIOR FINISH } \\
\text { (Corr. \& Exit) }\end{array}$ & & & & \\
\hline $\begin{array}{l}\text { 3. INTERIOR FINISH } \\
\text { (ROoms) }\end{array}$ & & & & \\
\hline $\begin{array}{l}\text { 4. CORRIDOR } \\
\text { PARTITIONS/WALLS }\end{array}$ & & & & \\
\hline $\begin{array}{l}\text { 5. DOORS TO } \\
\text { CORRIDOR }\end{array}$ & & & & \\
\hline 6. ZONE DIMENSIONS & & & & \\
\hline 7. VERTICAL OPENING & & & & \\
\hline 8. HAZARDOUS AREAS & & & & \\
\hline 9. SMOKE CONTROL & & & & \\
\hline $\begin{array}{l}\text { 10. EMERGENCY } \\
\text { MOVEMENT ROUTES }\end{array}$ & & & & \\
\hline $\begin{array}{l}\text { 11. MANUAL FIRE } \\
\text { ALARM }\end{array}$ & & & & \\
\hline $\begin{array}{l}\text { 12. SMOKE DETECTION } \\
\& \text { ALARM }\end{array}$ & & & & \\
\hline $\begin{array}{l}\text { 13. AUTOMATIC } \\
\text { SPRINKLERS }\end{array}$ & & & $\div 2=$ & \\
\hline TOTAL VALUE & $s_{1}=$ & $s_{2}=$ & $S_{3}=$ & $s_{G}=$ \\
\hline
\end{tabular}

Figure 23c. Fire/smoke zone evaluation work sheet for health care facilities 
Step 6: Determine Mandatory Safety Requirement Values - Use Table 6.

A. Using the classification of the building (i.e., New or Existing) and the floor where the zone is located, circle the appropirate value in each of the three columns in Table 6.

B. Transfer the three circled values from Table 6 to the blocks marked $S_{a}, S_{b}$, and $S_{c}$ in Table 7.

\begin{tabular}{|l|c|c|c|c|c|c|}
\hline Table 6. & \multicolumn{6}{|c|}{ MANDATORY SAFETY REQUIREMENTS } \\
\hline & \multicolumn{2}{|c|}{$\begin{array}{c}\text { CONTAINMENT } \\
\text { S }\end{array}$} & \multicolumn{2}{|c|}{$\begin{array}{c}\text { EXTINGUISHMENT } \\
\text { Sb }\end{array}$} & \multicolumn{2}{|c|}{$\begin{array}{c}\text { PEOPLE MOVEMENT } \\
\text { S }\end{array}$} \\
\hline ZONE LOCATION & New & EXist. & New & Exist. & New & Exist. \\
\hline FIRST FLOOR & 9.0 & 4.0 & 6.0 & 3.0 & 6.0 & 1.0 \\
\hline ABOVE FIRST FLOOR & 14.0 & 8.0 & 8.0 & 5.0 & 9.0 & 3.0 \\
\hline
\end{tabular}

Step 7: Evaluation Fire Safety Equivalency - Use Table 7.

A. Perform the indicated subtractions in Table 7. Enter the differences in the appropriate answer blocks.

B. For each row check "Yes" if the value in the answer block is zero or greater. Check "No" if the value in the answer block is a, negative number.

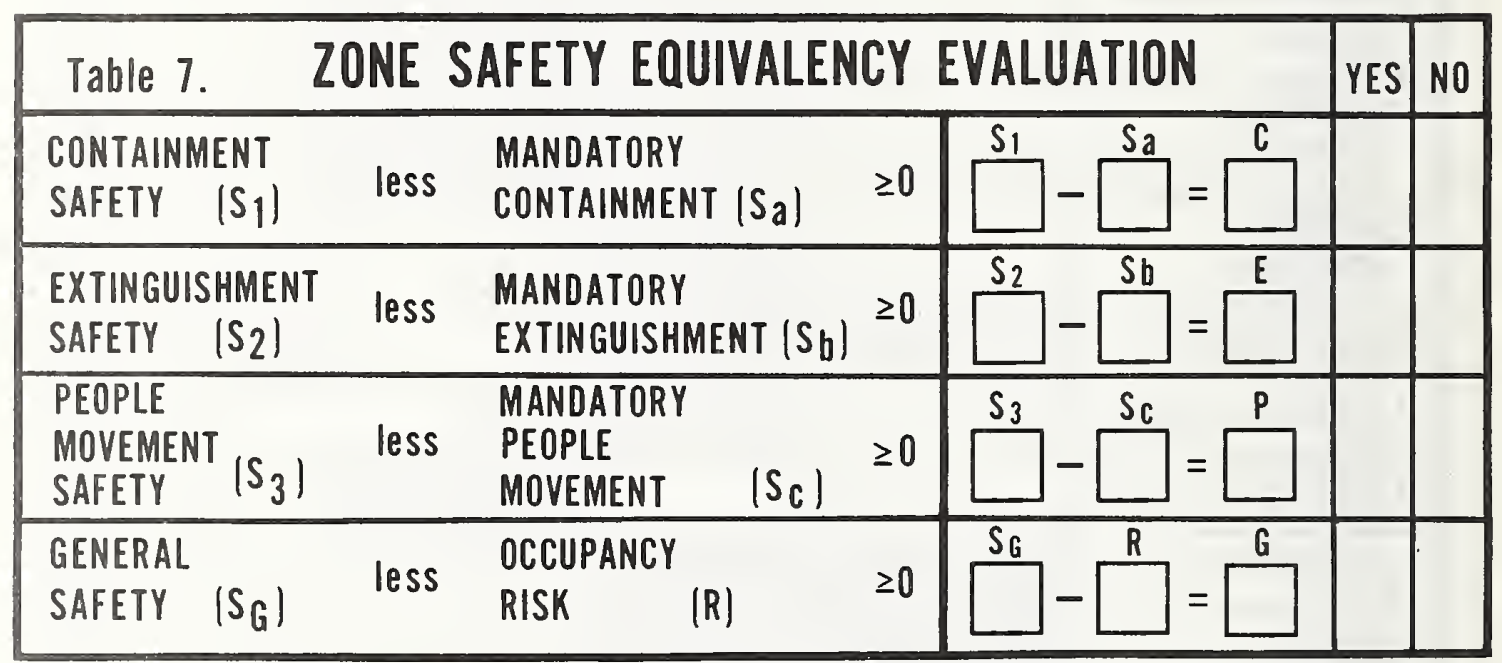

CONCLUSIONS:

1. [ ] All of the checks in Table 7 are in the "Yes" column. The level of fire safety is at least equivalent to that prescribed by the Life Safety Code*

2. [ ] One or more of the checks in Table 7 are in the "No" column. The level of fire safety is not shown by this system to be equivalent to that prescribed by the Life Safety Code."

-The equivalency covered by this worksheet includes the majority of considerations covered by the Life Safety Code. There are a few considerations that are not evaluated by this method. These must be separately considered. These additional considerations are covered in the "Facility Fire Safety Requirements Worksheet." One copy of this separate worksheet is to be completed for each facility.

Figure 23d. Fire/smoke zone evaluation work sheet for health care facilities 


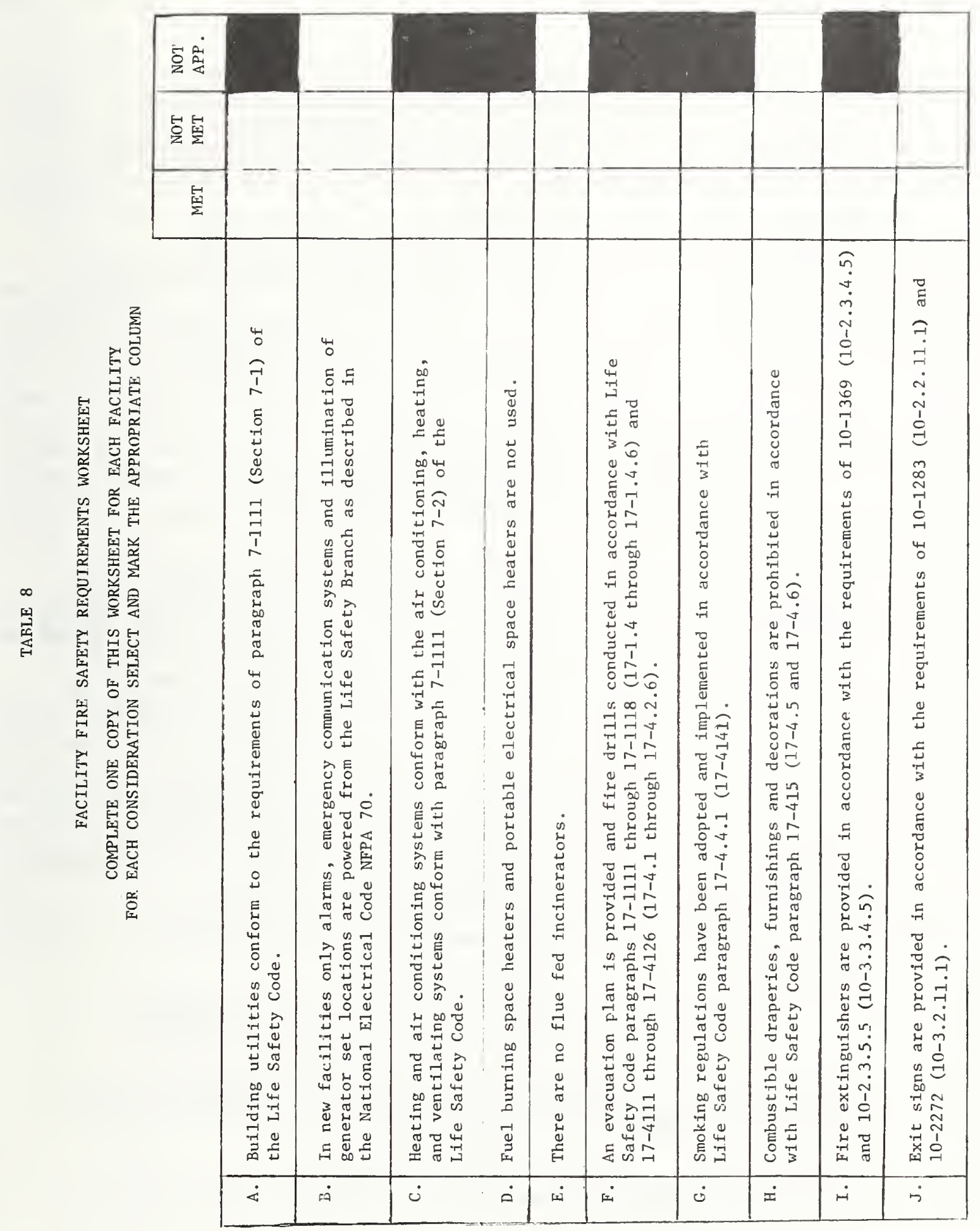

Figure 23e. Facility fire safety requirements work sheet 


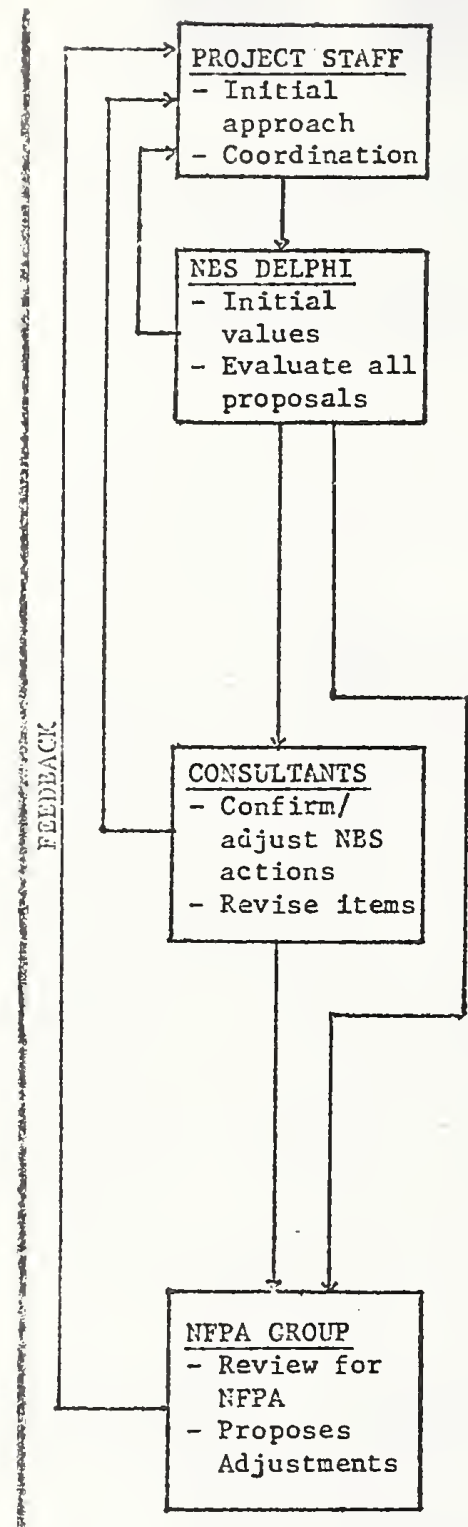

Project Staff

Harold E. Tielson, Fire Protection Engineer Program Chief

A. Jeffrey Shibe, Operations Research Analyst Michael J. Slifka, Fire Protection Engineer*

NBS Delphi Group

Irwin A. Benjamin, Structural Engineer Division Chief

Daniel Gross, Physicist, Deputy Division Chief Harold E. Nelson

Richard L.P. Custer, Fire Protection Engineer Program Chief

llarry Shoub, Fire Protection Engineer**

Bertram Vogel, Structural Engineer**

Michael J. Slifka*

Richard G. Bright, Fire Protection Engineer

Consultant Advisers

C. S. Mullen, Virginia State Fire Narshal

James Dowling, AIA Staff

J. Amand Eurgun, Chairman, MFPA Comittee on Safety to Life

David Iong, American Health Care Assoclation H. J. Werner, Research Director, DHUD (deceased) Harry T. Anderson, Chief, Fire Protection, Navy lichael J. Slifka, Chief, Safety and Protection, VA* Richard E. Stevens, Assistant Vice President, IFPA Joseph Sprague, American Iospital Association Jonas Norehart, Eire Protection Engineer, DHEW Robert M. Suckling, Architect, AIA Comittee on Architecture for Healtin

Thomas Imbs, Architect, AIA Comittee on Codes and Standards

William A. Schmidt, Research Engineer, VA David Boor, JCAH, Fire Prot. Engineering Consultant Jack Sanders, Oklahora State Fire Marshal

NFPA Working Group (NFPA Comrittee on Safety to Life)

Allen Savadkin, DHEW

David Boor (Thomas Hanrahan), JCAH

Joseph Sprague, AHA

Thomas Jaeger, Fire Protection Consultant

Harold E. Nelson, NBS

A. Jeffrey Shibe, NBS

Arthur Bergantz, Fire Protection Consultant

David Elwing, Fire Protection Consultant

Donald Belles, Fire Protection Consultant

* Mr. Slifka was a member of the Program for Design Concepts (i.e. the Project Staff) unt1l 2/77. He then became Chief, Safety and Fire Protection for the Veterans Administration. Since then he has continued to participate in the Delphi Group and joined the Consultants Advisors.

*kir. Vogel succeed Mr. Shoub upon Mr. Shoub's retirenent. Both are experts In code administration and content.

Figure 24. Liaison with review groups 


\section{APPENDIX A \\ NBS DELPHI GROUP}

\section{Delphi Method}

The Delphi technique was developed in the 1950's for the purpose of estimating the probable effects of atomic bombing attacks on the United States. Since then it has been applied to technological forecasting as well as in areas where judgmental information is required. The Delphi technique is basically concerned with the utilization of the combined knowledge of experts to arrive at a consensus opinion where factual information is incomplete.

The NBS exercise followed a process called Policy Delphi. The basic premise of the Policy Delphi is that it acts as a precursor to a committee activity. The Policy Delphi is not a substitute for research studies, analyses, or staff work. It is, however, an organized method for correlating views and information pertaining to a specific problem area and for allowing the respondents representing such views and information the opportunity to react to and assess differing viewpoints. Because the respondents are anonymous, fear of potential repercussions or embarrassment is removed and no simple individual need commit himself publicly to a particular view until after the alternatives have been put on the table.

Turoff in "The Policy Delphi"* analyzed committee and Delphi processes. The study points out that a Delphi followed by a committee provides good results in formulating policies.

The study identifies two major areas of problems with large size committees (i.e. communication and psychological). The communication difficulties are attributed to the diverse membership. The major lack of understanding tends to be between the following groups: individuals who are not familiar with many of the new decision aids coming out of operation research and system analyses but who have an intuitive feel for the complexities of the organization, and individuals who have been trained in many of modern management techniques and who are sometimes a little too confident that these approaches can be applied to every problem. The problems associated with operation of committees that tend to reflect psychological characteristics are:

- The domineering personality, or outspoken individual that takes over committee process.

- The unwillingness of individuals to take a position on an issue before all facts are in or before it is known which way the majority is headed.

- The difficulty of publicly contradicting individuals in higher positions.

*Murray Turoff, "The Design of a Policy Delphi," Technological Forcasting and Social Changes 2, No. 2 (1970). 
- The unwillingness to abandon a position once it is publicly taken.

- The fear of bringing up uncertain ideas that may turn out to be idiotic and results in loss of face.

The above limitation may also apply to small size committees, except when the members of the small committee are given sufficient time to consider and explore the issue, and have assurance that the privacy of their respective remarks will be respected outside the committees. Under those conditions a small committee will not have any of the difficulties which have been identified for the large size committee.

Usually Delphi, whether it is to be conventional or computerized, undergoes four distinct phases. The first phase is characterized by exploration of the subject under discussion, wherein each individual contributes additional information he feels is pertinent to the issue. The second phase involves the process of reaching an understanding of how the group views the issue. If there is significant disagreement among members, the disagreement is explored in the third phase to bring out the underlying reasons for differences and possibly to evaluate them. The last phase, a final evaluation, occurs when all previously gathered information has been initially analyzed and the evaluations have been fed back for consideration.

There are two methods of gaining consensus: conventional and computerized. In the conventional form, a monitor team designs a questionnaire which is sent to a respondent group. After the questionnaire is returned, the monitor team summarizes the results, and based upon the results develops a revised questionnaire for the respondent group to answer. The respondent group is usually given at least one opportunity to revise its original answers after examining the group response.

The computerized method replaces the monitor group to a large degree with a computer which has been programmed to carry out the compilation of the respondent group results. This process has the advantage of eliminating delays in summarizing each round of Delphi, thereby turning the process into a real-time communication system. However, it does require that the information received from the respondents is in a form that can be fed into a computer and that an algorithm can be provided to analyze the data. The NBS Delphi Group used the conventional fourphase approach in its evaluation process.

Approach Used in Developing Fire Safety Parameters and Their Values

\section{CFR Delphi Group}

Seven individuals from the Engineering Division of the Center for Fire Research were chosen to act as a "Delphi" group. The experience of the group members in areas of fire/life safety ranged from six to thirty-five years. (Table A-1 identifies the group members.) Each individual was briefed about the general nature of the life safety risk A-2 
analysis system and was given a detailed description of the safety model. The individuals were encouraged to seek more information about the system or any individual parameter, if the information given to them was insufficient. No guidance was provided as to the importance of any sub-model or individual parameter.

Instructions for Completing of Forms

Each member of the group was given four separate but identical forms, one for each of four fire safety functions: 1) containment safety; 2) extinguishment safety; 3) people movement safety; 4) general safety, (see figure A-1). He was told that the safety requirements should be considered only as they apply to health care facilities. He was also instructed that the safety impact of each requirement should be established on a fire/smoke zone basis. Specific instructions for filling out the questionnaire were:

1. Evaluate the relative worth of the safety requirement, (i.e. parameter) on each of the four fire safety functions, one function at a time, and record conclusions on the appropriate questionnaires.

2. Use numerical values to express the level of safety for each subdivision of each parameter.

3. The numerical values should range from $(+10)$ for the highest level of safety to $(-10)$ for the condition creating the most severely hazardous condition. Where the parameter status neither provides safety nor creates a hazardous condition, a "zero" value should be assigned.

4. Add additional safety parameters to any of the questionnaires, if required to provide a more complete safety evaluation.

5. Increase the number of parameter subdivisions, if the number shown on the forms is insufficient.

6. Remarks may be made on each of the four forms.

Analysis of the Questionnaires

1. Forms. The completed forms were checked for completeness, illegible numbers and remarks. Where required, individuals were asked to provide additional information to complete the questionnaire. Figure A-2 shows the format agreed to by the Delphi group.

2. Preparation of Parameter Values. Some individuals did not use the entire range of suggested numbers ( +10 to -10$)$. Therefore, the set of numbers from their forms was normalized to be compatible with the other forms. Each individual was requested to submit 216 values, which made the process of adjustments quita laborious. The values for each safety level were clustered to identify where major deviations occurred. An individual who supplied values significantly different from the cluster was asked for the reasoning behind his choice. Mostly the 
differences were generated by misinterpretations of the safety parameter functions. The values were then adjusted by the individuals and the process of preparing a consensus safety parameter table began.

3. Safety Parameter Table. After adjustment of the parameter values as described above a single table of safety parameters was prepared using the arithmetic means (see figure A-3). All the values were expressed as whole numbers rounded off toward the "conservative" side.

\section{Safety Parameter Selection for the Three Sub-models. It was} recognized that al1 of the selected parameters were not important in providing safety in a particular fire safety function. Based on the response of the group, a process was developed to assign only those parameters which provide significant values in evaluating a prescribed life safety function. The three functions are: containment, extinguishment, and people movement safety. (There is also the general safety function). For each of the three functions a set of three tables was prepared. The first table had all the values of each parameter as assigned by the individual respondent. The second table was similar to the first except numerical values were clustered in six ranks as follows:

High (10-8); Medium (7-4); Low (3-0); Negative Low ( -1 to -3 ); Negative Medium ( -4 to -7$)$; Negative High $(-8$ to -10$)$. The third table ranked the parameters, according to whether they provided maximum or minimum safety. Based on the information from the third table, safety parameters were chosen for each of the sub-models. Seven parameters were assigned to the containment safety sub-model; five parameters were assigned to the extinguishment safety; and seven parameters were assigned to the people movement safety. (See figure A-4).

5. Results. The analysis of the questionnaire filled out by the Delphi respondents provided the basis for assigning numbers to each subdivision of each parameter as described above, assigning parameters to the safety function; and dropping or changing parameters. The group did not assign sufficient positive or negative values to two proposed safety parameters (ratio of patients to attendants in the zone, and emergency brigades in health care facility). Therefore, the parameter on the brigades was dropped from future group evaluation; and, the parameter on the ratio of patients to attendants in the zone was transferred to the risk model (see figure 23-A). The group suggested that the construction safety parameter be broken into more categories, to be more meaningful as well as to reflect better conformance with the Life Safety Code. The results were summarized in new tables which were presented to group meetings. The group discussed the proposed forms and suggested additional changes. The construction safety parameter was reorganized and presented to individual members for evaluation as well as for the assignment of values for each level of safety. The values were analyzed and a single set of values was generated and included in the safety parameters table.

6. Adjustments. The Delphi group was instructed to make evaluations on the merit of the safeguards being judged without reference to A-4 
specific requirements of the Life Safety Code. The initial safety parameter table contained negative values for several parameter levels that met requirements of the Life Safety Code (fire resistive partitions, smoke detection, corridor flame spread). The group made a decision that these parameter levels should have at least a "zero" safety value. These negative parameter values (for the specific code requirements) were then adjusted to the "zero" value. Each parameter which had a level upgraded was then adjusted so that all of the levels of that parameter reflected the necessary changes.

7. Delphi Group Status. The Delphi group finished its prime assignment to provide the basic system to be analyzed by the outside consultant group. The Delphi group also met several times after finishing this initial assignment, to consider adjustments or changes to the system suggested by the outside consultants or identified through NBS research. At each meeting the group analyzed the problem and suggested possible improvements to the system. 
Table A-1

NBS Delphi Group

Benjamin, Irwin

Gross, Daniel

Custer, Richard

Nelson, Harold

Bright, Richard

Slifka, Michael

Shoub, Harry*

Vogel, Bertram*
Division Chief, Structural Engineer

Deputy Division Chief, Mechanical Engineer

Program Chief, Fire Protection Engineer

Program Chief, Fire Protection Engineer

Fire Protection Engineer

Fire Protection Engineer

Fire Protection Engineer

Codes Specialist

*Mr. Vogel succeeded Mr. Shoub on the latter's retirement. 


\begin{tabular}{|c|c|c|c|c|c|c|c|}
\hline \multirow{3}{*}{ 1. CONSTRUCTION } & \multicolumn{4}{|c|}{ COMBUSTIBLE } & \multirow{2}{*}{\multicolumn{3}{|c|}{ NON COMBUSTIBLE }} \\
\hline & \multicolumn{2}{|c|}{ WOOD FRAME } & \multicolumn{2}{|c|}{ ORDINARY } & & & \\
\hline & \begin{tabular}{|l|l|} 
UNPROTECTEO & PR \\
\end{tabular} & ROTECTEO & UNPROTECTEO & \begin{tabular}{|l|l|l|} 
EO & PROTECTEO & UNP \\
\end{tabular} & UNPROTECTEO & PROTECTEO & FIRE RESISTIVE \\
\hline \multirow{2}{*}{$\begin{array}{l}\text { 2. FLAME SPREAD } \\
\text { (Corr.\& Exit) }\end{array}$} & \multirow[t]{2}{*}{ CLASS C } & \multicolumn{2}{|c|}{ CLASS B } & CLASS A & & & \\
\hline & & & & & & & \\
\hline \multirow{2}{*}{$\begin{array}{l}\text { 3. FLAME SPREAD } \\
\text { (Rooms) }\end{array}$} & CLASS C & \multicolumn{2}{|c|}{ CLASS B } & \multirow[t]{2}{*}{ CLASS A } & & & \\
\hline & & & & & & & \\
\hline \multirow{2}{*}{$\begin{array}{l}\text { 4. FIRE RESISTIVE } \\
\text { (Partition) }\end{array}$} & \multirow[t]{2}{*}{$<1 / 3 \mathrm{HR}$} & \multirow{2}{*}{\multicolumn{2}{|c|}{$1 / 3-1.0 \mathrm{HR}$}} & \multirow[t]{2}{*}{$>1.0 \mathrm{HR}$} & & & \\
\hline & & & & & & & \\
\hline \multirow{2}{*}{$\begin{array}{l}\text { 5. FIRE RESISTIVE } \\
\text { (Vertical Exits) }\end{array}$} & \multirow[t]{2}{*}{$<1.0 \mathrm{HR}$} & \multirow{2}{*}{\multicolumn{2}{|c|}{$1.0-2.0 \mathrm{HR}$}} & \multirow[t]{2}{*}{$>2.0 \mathrm{HR}$} & & & \\
\hline & & & & & & & \\
\hline \multirow{2}{*}{ 6. SMOKE CONTROL } & NO CONTROL & \multirow{2}{*}{\multicolumn{2}{|c|}{ STAIRS \& TOWER }} & \multirow[t]{2}{*}{ STAIRS \& CORRIDOR } & \multicolumn{2}{|c|}{ TOTAL SPACE } & \\
\hline & & & & & & & \\
\hline 7. HORIZONTAL EXITS & NO & \multicolumn{2}{|c|}{$\begin{array}{c}\text { YES W/O } \\
\text { SMOKE CONT. }\end{array}$} & $\begin{array}{l}\text { YES \& CORR. } \\
\text { SMOKE CONT. }\end{array}$ & & & \\
\hline 8. ALARM SYSTEM & N0>6 OCCUP. & $\mathrm{N} 0<6$ & $=60$ OCCUP. & $\begin{array}{l}\text { YES W/O F.0. } \\
\text { CONNECTION }\end{array}$ & $\begin{array}{l}\text { YES W } \\
\text { CONNE }\end{array}$ & $\begin{array}{l}\text { V.D. } \\
\text { CTION }\end{array}$ & \\
\hline MANUAL & & & & & & & \\
\hline & NO & & JRRIOOR & CORR.\& HABIT. & TOTALS & SPACES & \\
\hline 9. DETECTION SYSTEM & & & - & & & & . \\
\hline 10. DOOR OCCUP. & NO DOOR & $<20$ & MIN. FR. & $>20$ MIN. FR. & $\begin{array}{l}>20 \text { MIN. } \\
\text { SELF C }\end{array}$ & $\begin{array}{l}\text { FR. \& } \\
\text { CLOS. }\end{array}$ & \\
\hline TO CORRIDOR & & & & & & & \\
\hline & NONE & & RRIOOR & $\begin{array}{l}\text { CORR \& HABIT. } \\
\text { SPACE }\end{array}$ & TOTAL S & SPACES & \\
\hline II. SPRINKLERS & & & & & & & \\
\hline & $>100^{\prime}$ & & $5^{\prime}-100^{\prime}$ & $50^{\prime}-75^{\prime}$ & 50 & & \\
\hline 12. CORRIDOR LENGTH & & & & & & & \\
\hline RATIO OF OCCUP. & $>10$ TO 1 & & 10 T0 1 & $<5$ T0 1 & & & \\
\hline $\begin{array}{l}\text { 13. TO ATTENDANTS } \\
\text { PRESENT IN F. } Z \text {. }\end{array}$ & & & & & & & \\
\hline 14. FIRE FIGHTERS & $>10$ MIN. & & 0 MIN. & & & & \\
\hline PUBLIC & & & & & & & \\
\hline 15. EMERGENCY BRIGADE & $>20$ OCC. PER F.F. & \begin{tabular}{l|l} 
F. & $<200 \mathrm{OC}$ \\
\end{tabular} & CC. PER F.F. & & & & \\
\hline HEALTH FACILITY & & & & & & & \\
\hline
\end{tabular}

Figure A-1. Safety parameters - Delphi format 


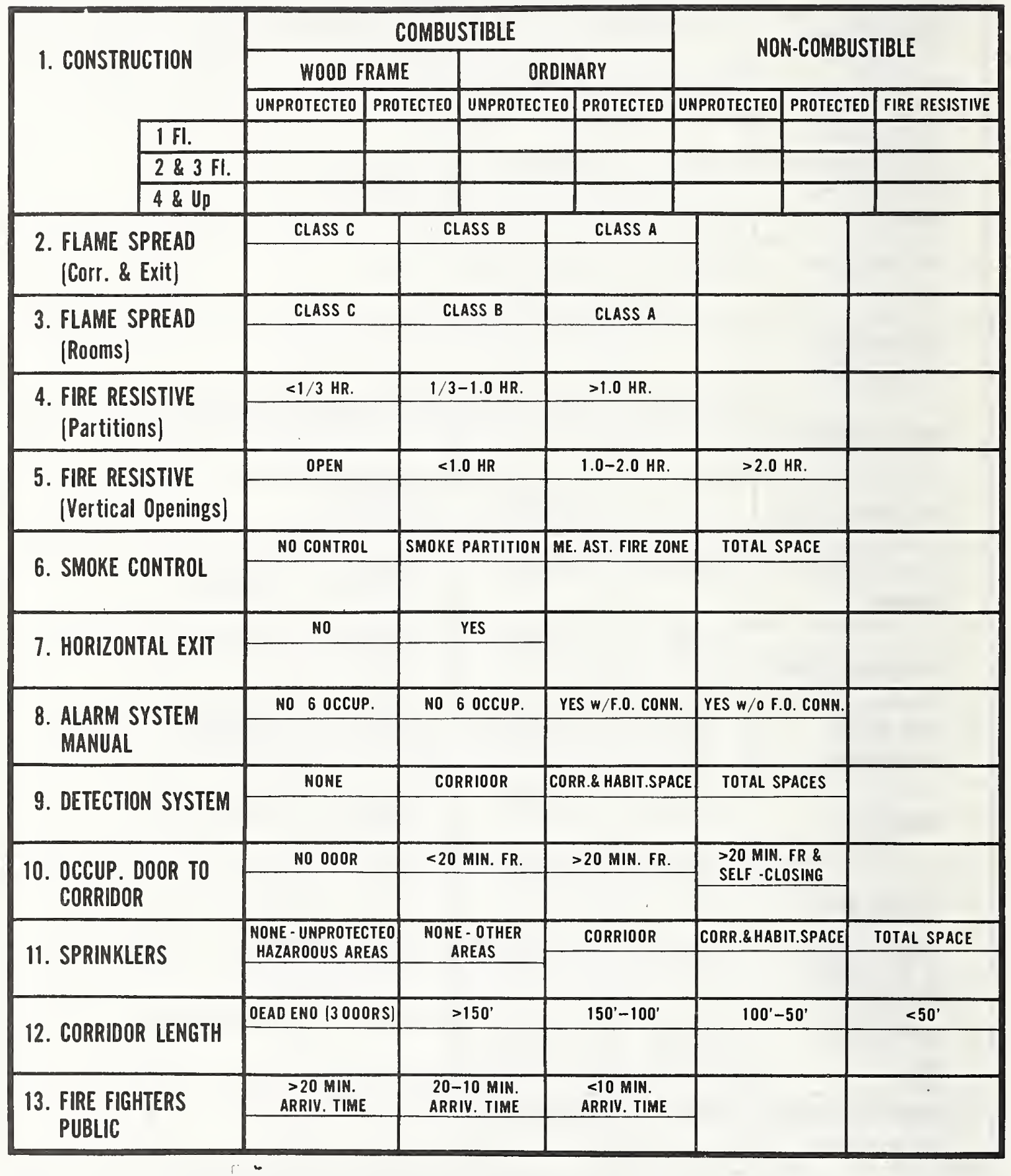

Figure A-2. Safety parameters matrix 


\begin{tabular}{|c|c|c|c|c|c|c|c|}
\hline \multirow{3}{*}{ 1. CONSTRUCTION } & \multicolumn{4}{|c|}{ COMBUSTIBLE } & \multirow{2}{*}{\multicolumn{3}{|c|}{ NON-COMBUSTIBLE }} \\
\hline & \multicolumn{2}{|c|}{ WOOD FRAME } & \multicolumn{2}{|c|}{ ORDINARY } & & & \\
\hline & \begin{tabular}{l|l} 
UNPROTECTEO & PR \\
\end{tabular} & PROTECTED & UNPROTECT & \begin{tabular}{|l|l|l} 
TEO & PROTEC TEO & UN \\
\end{tabular} & UNPROTECTEO & PROTECTE & FIRE RESISTIVE \\
\hline $1 \mathrm{Fl}$. & \begin{tabular}{l|l}
-2 & \\
\end{tabular} & 0 & -2 & \begin{tabular}{l|l|l} 
& 0 & \\
\end{tabular} & 0 & 2 & 2 \\
\hline $2 \& 3 \mathrm{Fl}$. & -8 & -2 & -8 & -2 & -2 & 2 & 4 \\
\hline $4 \&$ Up & -10 & -6 & -10 & -6 & -4 & 2 & 4 \\
\hline \multirow{2}{*}{$\begin{array}{l}\text { 2. FLAME SPREAD } \\
\text { (Corr. \& Exit) }\end{array}$} & CLASS C & \multicolumn{2}{|c|}{ CLASS B } & CLASS A & & & \\
\hline & -5 & \multicolumn{2}{|r|}{0} & 3 & & & \\
\hline \multirow{2}{*}{$\begin{array}{l}\text { 3. FLAME SPREAD } \\
\text { (Rooms) }\end{array}$} & CLASS C & \multicolumn{2}{|c|}{ CLASS B } & CLASS A & & & \\
\hline & -3 & \multicolumn{2}{|r|}{1} & 3 & & & \\
\hline \multirow{2}{*}{$\begin{array}{l}\text { 4. FIRE RESISTIVE } \\
\text { (Partitions) }\end{array}$} & $<1 / 3 \mathrm{HR}$. & \multicolumn{2}{|c|}{$1 / 3-1.0 \mathrm{HR}$. } & $>1.0 \mathrm{HR}$ & & & \\
\hline & 0 & & 2 & 3 & & & \\
\hline \multirow{2}{*}{$\begin{array}{l}\text { 5. FIRE RESISTIVE } \\
\text { (Vertical Openings) }\end{array}$} & OPEN & \multicolumn{2}{|c|}{$<1.0 \mathrm{HR}$} & $1.0-2.0 \mathrm{HR}$. & \multicolumn{2}{|c|}{$>2.0 \mathrm{HR}$. } & \\
\hline & -5 & \multicolumn{2}{|r|}{0} & 3 & \multicolumn{2}{|c|}{4} & \\
\hline & NO CONTROL & SMOKE & PARTITION & ME. AST. FIRE ZONE & TOTAL S & PACE & \\
\hline 6. SMUKE CONIRUL & $-2^{*}$ & & 0 & 3 & 4 & & \\
\hline 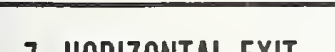 & N0 & & YES & & & & \\
\hline I. HURILUNIAL EXII & 0 & & 3 & & & & \\
\hline 8 ALARM SYSTFM & NO 6 OCCUP. & NO & 6 OCCUP. & YES W/F.O. CONN. & YES W/OF. & 0. CONN. & \\
\hline MANUAL & -4 & & 0 & 1 & 2 & & \\
\hline O DETС & NONE & & RRIDOR & CORR.\& HABIT.SPACE & $\begin{array}{ll}\text { E.E. AST.B } \\
\end{array}$ & Y ROOM & \\
\hline 9. UEIELTIUN STSIEM & 0 & & 2 & 4 & 5 & & \\
\hline 10. OCCUP. DOOR TO & NO OOOR & $<20$ & MIN. FR. & $>20$ MIN. FR. & $\begin{array}{l}>20 \text { MIN. } \\
\text { SELF }- \text { CLO }\end{array}$ & $\begin{array}{l}\text { FR \& } \\
\text { OSING }\end{array}$ & \\
\hline CORRIDOR & -5 & & 0 & 1 & 4 & & \\
\hline 11. SPRINKLERS & $\begin{array}{l}\text { MONE - UNPROTECTEO } \\
\text { HAZAROOUS AREAS }\end{array}$ & & $\begin{array}{l}\text { E-OTHER } \\
\text { AREAS }\end{array}$ & CORRIOOR & CORR.\&HABI & IT.SPACE & TOTAL SPACE \\
\hline & -3 & & 0 & 5 & 8 & & 10 \\
\hline & OEAO ENO [3 DOORS & & $>150^{\prime}$ & $150^{\circ}-100^{\prime}$ & $100^{\prime}-$ & & $<50^{\prime}$ \\
\hline 12. CURRIDUR LENGIH & -4 & & -2 & 0 & 1 & & 2 \\
\hline 13. FIRE FIGHTERS & $\begin{array}{l}>20 \text { MIN. } \\
\text { ARRIV. TIME }\end{array}$ & & $\begin{array}{l}-10 \text { MIN. } \\
\text { RIV. TIME }\end{array}$ & $\begin{array}{l}<10 \text { MIN. } \\
\text { ARRIV. TIME }\end{array}$ & & & \\
\hline PUBLIC & -5 & & 0 & 2 & & & \\
\hline
\end{tabular}

NOTE : *Fire zones with less than 30 people substitute $[-2]$ with $[0.0]$

Figure A-3. Safety parameters values

$$
\text { A-9 }
$$




\begin{tabular}{|c|c|c|c|c|}
\hline $\begin{array}{c}\text { SAFETY } \\
\text { PARAMETERS }\end{array}$ & $\begin{array}{c}\text { GENERAL } \\
\text { SAFETY } \\
\left(S_{G}\right) \\
\end{array}$ & $\begin{array}{l}\text { CONTAINMENT } \\
\text { SAFETY } \\
\left(S_{1}\right) \\
\end{array}$ & $\begin{array}{c}\text { EXTINGUISHMENT } \\
\text { SAFETY } \\
\left(S_{2}\right) \\
\end{array}$ & $\begin{array}{c}\text { PEOPLE } \\
\text { MOVEMENT } \\
\text { SAFETY }\left(S_{3}\right)\end{array}$ \\
\hline 1. CONSTRUCTION & & & & \\
\hline $\begin{array}{l}\text { 2. FLAME SPREAD } \\
\text { (Corr. \& Exit) }\end{array}$ & & & & \\
\hline $\begin{array}{l}\text { 3. FLAME SPREAD } \\
\text { (Rooms) }\end{array}$ & & & & \\
\hline $\begin{array}{l}\text { 4. FIRE RESISTIVE } \\
\text { (Partitions) }\end{array}$ & & & & \\
\hline $\begin{array}{l}\text { 5. FIRE RESISTIVE } \\
\text { (Vertical Openings }\end{array}$ & & & & \\
\hline 6. SMOKE CONTROL & & & & 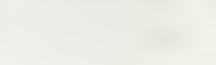 \\
\hline 7. HORIZONTAL EXIT & & & & \\
\hline $\begin{array}{l}\text { 8. ALARM SYSTEM } \\
\text { MANUAL }\end{array}$ & & & & \\
\hline 9. DETECTION SYSTE & & & & \\
\hline $\begin{array}{l}\text { 10. OCCUP. DOOR TO } \\
\text { CORRIDOR }\end{array}$ & & & & \\
\hline 11. SPRINKLERS & & & & \\
\hline 12. CORRIDOR LENGTH & & & & \\
\hline $\begin{array}{l}\text { 13. FIRE FIGHTERS } \\
\text { PUBLIC }\end{array}$ & & & & \\
\hline .TOTAL VALUE & & & & \\
\hline
\end{tabular}

Figure A-4. Individual safety evaluations 


\section{APPENDIX B \\ CONSULTANT GROUP}

The Consultant Group consisted of prominent persons in the regulation or specification of fire safety for health care facilities. This included regulatory officials, code writing officials, hospital surveyors, government agency fire protection chiefs, and accrediting officials representing a cross section of the applied field. The group membership is shown in Table B-1. The dates of meetings and attendance are shown in Table B-2.

Table B-1 Consultant Group

Name

Anderson, H. T.

Morehart, J.

Schmidt. W. A.

Scott, H.

Slifka, M. J.

Werner, W. J.

(deceased)

Guest, J .

Mullen, C. S.

Sanders, J.
Affiliation

Naval Facility Engineering

Command

Chief, Fire Protection

Navy

Department of H.E.W.

Fire Safety Engineer

Office of the Secretary

Veterans Administration.

Research Engineer

Veterans Administration

Fire Safety Engineer

Veterans Administration

Chief, Safety \& Protection

Department of H.U.D.

Research Director

Policy Development \& Research

Deputy Fire Marshal

State of Oklahoma

Fire Marshal

State of Virginia

Fire Marshal

State of Oklahoma
Representative

Risk Management

Risk Management

Researcin

Risk Management

Risk Management

Regulatory

Code Enforcing

Code Enforcing

Code Enforcing 


\begin{tabular}{|c|c|c|}
\hline Walsh, M. & $\begin{array}{l}\text { Cincinnati Ohio } \\
\text { Director, Buildings \& } \\
\text { Inspections Department }\end{array}$ & Code Enforcing \\
\hline Boor, D. & $\begin{array}{l}\text { Loss Control Services } \\
\text { Fixe Safety Engineer }\end{array}$ & Accreditation \\
\hline Elwing, D. & $\begin{array}{l}\text { Joint Commission on } \\
\text { Accreditation of Hospitals } \\
\text { (J.C.A.H.) }\end{array}$ & Accreditation \\
\hline Hanrahan, $T$. & $\begin{array}{l}\text { J.C.A.H. } \\
\text { Fire Safety Engineer }\end{array}$ & Accreditation \\
\hline Hoover, A. & $\begin{array}{l}\text { J.C.A.H. } \\
\text { Director }\end{array}$ & Accreditation \\
\hline Dowling, J. & $\begin{array}{l}\text { American Institute of } \\
\text { Architects }\end{array}$ & $\begin{array}{l}\text { Codes, American } \\
\text { Institute of } \\
\text { Architects (A.I.A.) }\end{array}$ \\
\hline Imbs, T. J. & Thomas Justin Imbs & Codes, A.I.A. \\
\hline Suckling, R. M. & $\begin{array}{l}\text { Rea, Hayes, Lange \& } \\
\text { Suckling }\end{array}$ & Health Design, A.I.A. \\
\hline Stevens, R. E. & $\begin{array}{l}\text { National Fire Protection } \\
\text { Assn. } \\
\text { Assistant Vice President }\end{array}$ & NFPA Life Safety Code \\
\hline Burgun, J.A. & Rogers, Butler \& Burgun & NFPA Life Safety Code \\
\hline Sprague, I. & American Hospital Assn. & Hospital Operators \\
\hline Long, D. & $\begin{array}{l}\text { American Health Care Assn. } \\
\text { Director of Life Safety } \\
\text { Programs }\end{array}$ & Health Care Operators \\
\hline
\end{tabular}


Table B-2. Dates of Meetings and Attendance

Neeting

Septembex 8-9, 1976

Washington, D.C.

Attendance

Anderson, $\mathrm{H}$. Burgun, $A$. Dorvling, J. Elwing, $D$.

\author{
Imbs, $\mathrm{T}$. \\ Morehart, J. \\ Mullen, S.
}

\section{Meeting}

November 18, 1976

Cincinnati, Ohio

Burgun A.

-Dowling, J.

E.lwing, D.

Hanrahan, $T$.

Imbs, T.

Burgun, A.

Hoover, $A$.

Imbs, $T$.

\section{Morehart, I. \\ Mulien, S. \\ Sanders, J. \\ Schmidt, $\mathrm{N}$.}

\section{Meeting}

June 13-14, 1977

Washington, D.C.

\section{Attendarise}

Norehart, J.
Nillen, S.
Slifka, M.
Meeting

March 1, 1978

Washington, D.C.

\section{Attendance}

Anderson, $\mathrm{H}$.

Boor, D.

Purgun, $A$.

Dowling, $J$.
Elwing, $D$.

Imbs, $T$.

Norehart, I.
Sanders, J.

Schmidt, $\mathrm{N}$.

Scott, $H$.

Slifka, M. Sprague, $J$. Stevens, R. Suckiing, $R$.
Sprague, $J$. suckling, $k$. Verner, $W$.
Nullen, s. Sprague, $J$. Suckling, $k$. 



\section{APPENDIX C}

CROSS CORRELATION BETWEEN EVALUATION SYSTEM AND THE LIFE SAFETY CODE

Definitions for hazards and safety used in the Fire/Smoke Zone Evaluation Work Sheet for Health Care Facilities (FSEW) were directly correlated to similar definitions used in the Life Safety Code.* This appendix provides a general cross reference between the FSEW and the Life Safety Code. Table C-1 shows the cross correlation for the definitions used in the FSEW and the Life Safety Code NFPA 101-1973.

The following code is being used to refer to the definitions used in the FSEW:
N.A.
Not Applicable
Numbers 1 to 13
FSEW, Table 4, Column 1
Parameters as listed in
figure 23-b.
Letters A thru J
FSEW, Table 8, Column 1
(figure 23-e of this report)

*Chapters 17 and 10 reproduced by permission from NFPA No. 101-1973

(Life Safety Code), copyright 1973, National Fire Protection Association, Boston, Massachusetts. 
TABLE $\quad-1$

CROSS REFERENCE TO THE LIFE SAFETY CODE (LSC) - 1973

\begin{tabular}{l|l} 
Evaluation* & LSC-1973 \\
Worksheet & L.A. \\
\hline 10-0001. Institutional buildings are those used for purposes such \\
as medical or other treatment or care of persons suffering from \\
physical or mentarilness, disease or tnfirmity; for the care of in- \\
fants, convalescents or aged persons; and for penal or corrective \\
purposes. Institutional buildings provide sleeping facilities for the \\
occupants and are occupied by persons who are mostly incapable \\
of self-preservation because of age, physical or mental disability, \\
or because of security measures not under the occupants' control. \\
Buildings or sections of buildings which house, or in which care is \\
rendered to mental patients who are capable of average judgment \\
in taking action for self-preservation under emergency conditions, \\
in the opinion of competent medical authority approved by the \\
state agency having jurisdiction, may come under other sections of \\
this Code instead of Section 10-1. \\
Sections of institutional buildings may come under other occu- \\
pancy classifications regarding exit requirements if these areas are \\
not used to house institutional occupants, or are not areas in which \\
these persons are treated or to which they have normal access, or \\
which serve as a means of egress for them. \\
Institutional buildings comprise three groups: groups (a) and (b) \\
are treated together in Chapter 10, and group (c) is considered \\
separately: \\
(a) Health Care Facilities (Hospitals and nursing homes) \\
(b) Residential-Custodial Care (Nurseries, homes for the aged, \\
mentally retarded care institutions, etc.) \\
(c) Residential-Restrained Care (Penal institutions, reforma- \\
tories, jails, etc.) See Section 10-3.
\end{tabular}

N.A.

10-0002. Institutional occupancies shall include all buildings. or parts thereof with occupancy as described in 10-0001. 
N.A.

N.A.

N.A.
10-0003. All institutional buildings shall be so designed, constructed, maintained, and operated as to minimize the possibility of a fire emergency requiring the evacuation of occupants. Because the safety of sccupants of institutional buildings cannot be assured adequately by dependence on evacuation of the building, their protection from fire shall be provided by appropriate arrangement of facilities, adequate staffing, and careful development of operating and maintenance procedures composed of the following:

(a) Proper design, construction, and compartmentation;

(b) Provisions for detection, alarm, and extinguishment; and

(c) Fire prevention and the planning, training, and drilling in programs for the isolation of fire and transfer of occupants to areas of refuge or evacuation of the building.

10-0004. It is recognized that in buildings housing various types of psychiatric paticnts, or used as penal institutions, it may be necessary to lock doors and bar windows that are equipped to confine and protect building inhabitants. Other sections of this Code requiring the kecping of exits unlocked may be waived by the authority having jurisdiction. It is also recognized that some psychiatric patients are not capable of sceking safety without guidance. In buildings in which doors arc locked or windows are barred, provisions shall be made for the rapid removal of occupants by such reliable means as the remote control oí locks or by keying all locks to kcys carried by attendants.

\section{0-0005. Definitions}

(a) Hospital - a building or part thereof used for the medical, psychiatric, obstetrical or surgical care, on a 24-hour basis, of 4 or more inpatients. Hospital, wherever used in this Code, shall include general hospitals, mental hospitals, tuberculosis hospitals, children's hospitals, and any such facilities providing inpatient care.

(b) Nursing Home - a building or part thereof used for the lodging, boarding and nursing care, on a 24-hour basis, of 4 or more persons who, because of mental or physical incapacity, may be unable to provide for their own needs and safety without the assistance of another person. Nursing home, wherever used in this Code, shall include nursing and convalescent homes, skilled nursing facilities, intermediate care facilities, and infirmaries of homes for the aged.

(c) Residential-Custodial Care Facility - a building, or part thereof, used for the lodging or boarding of 4 or more persons who are incapable of self-preservation because of age, or physical or mental limitation. This includes facilities such as homes for the aged, nurscries (custodial care for children under 6 years of age), and mentally retarded care institutions. Day care facilities that do not provide lodging or boarding for irstitutional occupants are not covered in this section of the Code. 
N.A.

1.

1.

1.

1.

1.

\section{SECTION 10-1. NEW HOSPITALS, NURSING HOMES AND RESIDENTIAL-CUSTODIAL CARE OCCUPANCIES}

\section{0-111. Application}

10-1111. This Seetion establishes life safety requirements for hos. pitals, nursing homes, and residential-eustodial care institutionsWhere requirements varv. the speeifie oecupancy, such as hospital, nursing home, nursery, residential-custodial care institution, home for the aged, or mentally retarded care institution, is named in the paragraph pertaining thereto. See Chaplet 17 for operating fealures.

\section{0-112. New Construction, Additions, Conversions}

10-1121. Any addition shall be separated from any existing nonconforming strueture by a noneombustible fire partition having at least a 2-hour fire resistanee rating. Communieating openings in such dividing fire partition shall occur only in corridors and shall be proteeted by an approved self-closing fire door. Exeept where provisions meeting the requirements of 5-2134 and 10-1245 are made for such doors, they are intended normally to be kept elosed. Unless these doors are required exits, they are not required to swing with exit travel as speeified in 5-2121.

10-1122. Any building eonverted to these oecupaneies shall comply with all requirements for new facilities.

10-1123. See Section 2-2 for life safcty provisions during construction.

\section{0-113. Occupancy and Occupant Load}

10-1131. Institutional oceupaneies in buildings housing other oecupaneies shall be eompletely separated from them by noncombustible construetion having at least a 2-hour fire-resistanee rating. All means of egress from institutional oceupancies that traverse noninstitutional spaces shall eonform to requirements of this standard for institutional oeeupaneies. Any oecupancy with a hazard of contents elassified higher than that of the institution and loeated in the same building as institutional occupaneies shall be protected as required in Seetion 10-1371. Industrial, office, mereantile and storage oceupancies elassified as high-hazard shall not be permitted in buildings housing institutional oecupaneies.

10-1i32. Seetions of institutional buildings may be elassified as other oeeupaneies if they meet all of the following conditions:

(a) They are not intended to serve institutional oceupants for purposes of housing, treatment, eustomary access, or means of egress.

(b) They are adequately separated from areas of institutional occupaneies by eonstruction having a 2-hour fire resistanee rating. 
1.

1.

10.

10.

10.

10.
10-1133.* Auditoriums, chapels, stalt residential areas, garages or similar occupancies provided in connection with institutions shall have exits provided in accordance with other applicable sections of this Code.

10-1134. The occupant load for which means of egress shall be provided for any floor shall be the maximum number of persons intended to occupy that floor but not less than 1 person for each 120 square feet gross floor area in institutional sleeping departments and not less than 1 person for each 240 square fect of gross floor area of inpatient institutional treatment departments. Gross floor areas shall be measured within the exterior building walls with no deductions. (See Chapler 3.)

\section{0-12. Exit Details}

\section{0-121. Number and Types}

10-1211.* Exits shall be restricted to the following permissible types:
(a) Doors leading directly outside the building (see 10-124)
(b) Interior stairs and smokeproof towers (see 10-125)
(c) Ramps (see 10-127)
(d) Horizontal exits (see 10-126)
(e) Outside stairs (see Section 5-4)
(f) Exit Passageways (see Section 5-7)

10-1212. At least 2 exits of the above types, remote from each other, shall be provided for each floor or fire section of the building. At least 1 exit in each floor or fire section shall be as indicated in 10-1211 (a), (b), (c), or (f).

10-1213. Revolving doors shall not be counted as required exits, and shall not be installed except as specifically stated in Section 5-2.

10-1214. Elevators constitute a supplemetary facility, but shall not be counted as required exits. 


\begin{tabular}{|c|c|}
\hline $\begin{array}{l}\text { Evaluation* } \\
\text { Worksheet }\end{array}$ & LSC-1973 \\
\hline \multirow[t]{3}{*}{10.} & 10-122. Capacity of Exits \\
\hline & $\begin{array}{l}\text { 10-1221.* The capacity of any required exit shall be based on its } \\
\text { width in units of } 22 \text { inches as defined in } 5-115 \text {. The capacity of } \\
\text { exits providing travel by means of stairs shall be } 22 \text { persons per exit } \\
\text { unit; and exits providing travel without stairs, such as doors or } \\
\text { horizontal exits, shall be } 30 \text { persons per exit unit. }\end{array}$ \\
\hline & $\begin{array}{l}\text { Exception: The capacity of exits in Institutional Occupancies equipped } \\
\text { throughout with an approved automatic fire extinguishing system may be } \\
\text { increased to } 35 \text { persons per exit unit for travel by means of stairs, and } \\
\text { to } 45 \text { persons per exit unit for travel without stairs. }\end{array}$ \\
\hline
\end{tabular}

10.

\section{0-123. Access to Exit}

10-1231. Every aisle, passageway, corridor, exit discharge, exit location and access shall bc in accordance with Section 5-1, except as modified in the following paragraphs.

10.

10-1232. Travel distance (a) between any room door intended as exit access and an exit shall not exceed 100 fect; (b) between any point in a room and an exit shall not exceed 150 feet; (c) between any point in an institutional sleeping room or suite and an exit access door of that room or suite shall not exceed 50 feet. Travel distance shall be measured in accordance with 5-119.

Exception: The travel distances in $(a)$ or $(b)$ above may be increased by 50 feet in buildings completely equipped with an automatic. fre exlinguishing system.

10.

10-1233. Every institutional sleeping room, unless it has a door opening at ground level, shall have an exit access door leading directly to a corridor which leads to an exit. One adjacent room such as a sitting or anteroom may intervene if all doors along the path of exit travel are equipped with nonlockable hardware, except as provided in 10-1242, and this intervening room is not intended to serve more than 8 institutional sleeping beds.

Exception: Special nursing suites permitted in 10-1237 shall not be limited 108 beds or bassinets.

10.

10-1234. Aisles, corridors and ramps required for exit access or exit in a hospital or nursing home shall be at least 8 fect in clear and unobstructed width. Aisles, corridors and ramps required for exit access or exit in a residential-custodial care institution shall be at least 6 feet in clear and unobstructed width.

Exception: Corridors and ramps in adjunct areas not intended for the housing, treatment, or use of inpatients, may be a minimum of 6 feet in clear and unobstructed width. 
10.

6.

4.

10.

10.

10.
10-1235. Any room and any suite of rooms, as permitted in 10-1233, of more than 1,000 square feet shall have at least 2 exit access doors remote from each other.

10-1236. Every cxit or exit access shall be so arranged that no corridor or aislc has a pocket or dead end excecding 30 feet.

10-1237. Any institutional sleeping room which complies with the requirements previously set forth it this section may be subdivided with nonfire-rated, noncombustible partitions, provided, that the arrargement allows for direct and constant visual supervision by nursing personnel. Rooms which are so subdivided shall not exceed 5,000 square feet.

\section{0-124. Doors}

10-1241. Doors shall be in accordance with Section 5-2, except as modified below. For door requirements in horizontal exits and smoke partitions see 5-514, 6-6, 10-126, 10-131 and 10-231.

10-1242. Locks installed on institutional sleeping room doors shall be so arranged that they can be locked only from the corridor side. All such locks shall be arranged to permit exit from the room by a simple operation without the use of a key.

Exception No. 1: Doors leading directly to the outside of the building may be subject to locking from the room side.

Exception No. 2: Doors in homes for the aged may be lockable by the occupant if they can be unlocked from the opposite side, and keys are carried by attendants at all times.

Exception No. 3: Locks permitted by 10-0004.

10-1243. Exit access doors from hospital and nursing home sleeping rooms, diagnostic and treatment rooms or areas such as X-ray, surgery and physical therapy, all doors between these spaces and the required exits, and all exit doors serving these spaces shall be at least 44 inches wide. Doors to residential-custodial sleeping rooms and doors to nursery sleeping rooms and all exit doors serving these spaces shall be at least 36 inches wide.

Exception No. 1: Exit doors which are so located as not to be subject to use by any institutional occupant may be not less than 28 inches wide.

Exception No. 2: Doors in exit stair enclosures shall be not less than 36 inches wide. 


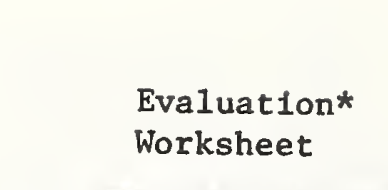

9. -10 .

8. -10 .

10.

10.

\section{LSC-1973}

10-1244.* Any door in a fire separation, horizontal exit or a smoke partition may be held open only by an electrical device which complies with 5-2134.

Each of the following systems shall be so arranged as to initiate the self-closing action, by zone or throughout the entire institutional occupancy:
(a) The required manual alarm system (10-1361), and (b) The required and approved automatic fire detection system
(10-1362), and
(c) An approved automatic fire extinguishing system, if provided.

10-1245. Doors in stair enclosures and in walls surrounding hazardous areas shall not be equipped with hold-open devices.

\section{0-125. Stairs, Smokeproof Towers}

10-1251. Every stair and smokeproof tower shall be in accordance with Section 5-3, shall be Class $A$, and shall be constructed as described in 10-1323.

Exception: Stairs that do not connect to a corridor, do not connect more than two levels and do not serve as a means of egress, need not comply
with these regulations.

\section{0-126. Horizontal Exits}

10-1261. * A horizontal exit shall be in conformance with Section 5-5 modified as below.

(a) At least 30 net square feet per occupant in a hospital or nursing home or 15 net square feet per occupant in a residential-custodial care institution shall be provided on each side of the horizontal exit for the total number of occupants in adjoining compartments.

(b) A single door may be used as a horizontal exit if it serves one direction only and is at least 44 inches wide for a hospital or nursing home or at least 36 inches wide for residential-custodial care institutions. The swing shall be in the direction of exit travel.

(c) A horizontal exit in a hospital or nursing home in a corridor 8 feet or, more in width serving as a means of egress from both sides of the doorway shall have the opening protected by a pair of swinging doors, each door to be a minimum of 44 inches wide and swinging in the opposite direction from the other.

(d) A horizontal exit in a residential-custodial care institution in a corridor 6 feet or more in width serving as a means of egress from both sides of the doorway shall have the opening protected by a pair of swinging doors, each door to be a minimum of 32 inches wide and swinging in the opposite direction from the other.

(e) An approved vision panel is required in each horizontal exit door. Center mullions are prohibited. 
10.

10-127. Ramps

10-1271. Ramps shall be in accordance with Section 5-6, and shall be Class $\mathrm{A}$ and shall not exceed 6 feet in vertical dimension between top and bottom floor elevations; a Class B ramp may be used where the height of the ramp is 1 foot or less. Ramp width shall be as specified in 10-1234.

B.

B.

J.

B.

9.

\section{0-128. Emergency Lighting, Exit Markings, Alarms and Com-} munication Systems

10-1281.* Each hospital shall be provided with emergency lighting as described in Section 5-10 and exit markings as described in Section 5-11. Such emergency lighting and the illumination of required exits and directional signs shall be supplied by the Life Safety Branch of the hospital elcctrical system as described in Chapter 3, NFPA 76A, (1973), Standard for Essential Electrical Systems for Hospitals. The Life Safety Branch shall also serve alarms, emergency communication systems and the illumination of generator set locations as described in paragraphs (c), (d) and (e), Section 312 of the same reference.

10-1282. Each nursing home and residential-custodial care facility shall have emergency lighting in accordance with Section 5-10.

Exception: Emergency lighting with at least one hour duration shall be provided.

10-1283. Exit signs shall be provided in each hospital, nursing home, and residential-custodial facility in accordance with Section $5-11$.

10-1284. Any alarm system(s) and any detection system(s) required in any institutional occupancy shall be provided with an alternative power supply in accordance with Section 220, NFPA $72 \mathrm{~A}$, Standard for the Installation, Maintenance, and Use of Local Protective Signaling Systems (1972).

\section{0-13. Protection}

\section{0-131.* Subdivision of Building Spaces}

10-1311. Smoke Partitions Required. Smoke partitions shall be provided, regardless of building construction type, as follows:

(a) To divide into at least two compartments every story used by inpatients for sleeping or treatment and any story having an occupant load of 50 or more persons (see 10-1134), and (b) To limit on any story the maximum area of each smoke com-
partment to no more than 22,500 square feet, of which both length or width shall be no more than $150 \mathrm{ft}$.

Exception: Protection may be accomplished in conjunction with the provisions of horizontal exits. 


\section{Evaluation* \\ Worksheet}

9.

9.

9.

9.

9.

9.

9.

1.

1.
10-1312. Smoke partitions shall be provided on stories which are usable but unoccupied.

10-1313. Any smoke partition sha!l be constructed in accordance with Section 6-6 and shall have a fire resistance rating of at least 1 hour.

10-1314. At least 30 net square feet per occupant for the total of bed or litter patients shall be provided on each side of the smoke partition. On other stories not housing bed or litter patients at least six net square feet per occupant shall be provided on each side of the smoke partition for the total number of occupants in adjoining compartments.

10-1315. Corridor openings in smoke partitions shall be protected by a pair of swinging doors, each door to swing in a direction opposite from the other. The minimum width of each door shall be as follows:

(a) Hospitals and nursing homes: 44 inches.

(b) Residential-custodial care institutions: 32 inches.

10-1316. Doors in smoke partitions shall comply with 6-613 and shall be self-closing and held open only if they meet the requirements of $10-1244$.

10-1317. Vision panels of approved transparent wired glass not exceeding 720 square inches in steei frames shall be provided in all doors in smoke partitions.

10-1318. Rabbets, bevels, or astragals are required at the meeting edges, and stops are required on the head and sides of door frames in smoke partitions. Positive latching hardware is not required. Center mullions are prohibited.

\section{0-132.* Minimum Construction Standards}

10-1321. Institutional buildings of 1 story in height only may be constructed of protected noncombustible construction, fire-resistive construction, protected ordinary construction, protected wood frame construction, heavy timber construction, or unprotected noncombustible construction. (See 10-136 for automatic sprinkler requirements.) For the purpose of 10-1321 and 10-1322, stories shall be counted starting at the lowest floor of exit discharge. All levels below the floor of exit discharge shall be separated from the floor of exit discharge by at least protected noncombustible construction.

10-1322. Institutional buildings 2 stories or more in height shall be of at least fire-resistive construction.

Exception: Institutional buildings up to and including three stories in height may be constructed of protected noncombustible construction if equipped throughout with an approved automatic extinguishing system. 
7.

1.

N.A.

4.

10-1323. Institutional occupancies two or more stories in height shall have enclosure walls of noncombustible materials having a fire resistance rating of at least two hours around stairways, elevators, chutes, and other vertical openings between floors. For other exceptions, see 10-1251.

Exception: The fire resistance rating of enclosures in institutional occupancies equipped throughout with an approved automatic extinguishing system may be reduced to one hour in buildings up 10, and including three stories.

10-1324. All interior walls and partitions in buildings of fire: rcsistive and noncombustible construction shall be composcd of noncombustible materials.

10-1325.* Every institutional sleeping room shall have an outside window or outside door arranged and located so that it ean be opened from the inside without the use of tools or keys to permit the venting of products of eombustion and to pernit any occupant to have direct aecess to fresh air in case of emergency. (See 10-0004 for detention screen requirements.) The maximum allowable sill height shall not exceed 36 inches above the floor.

Exception No. 1: The window sill in special nursing care areas may be 60 inches above the floor.

Exception No. 2: Rooms intended for occupancy of less than 24 hours, such as those housing obstetrical labor beds, recovery beds, observation beds in the emergency department and newborn nurseries, need not comply with this section.

\section{0-133. Construction of Corridor Walls.}

10-1331*. Corridors shall be separated from use areas by partitions having a fire-resistive rating of at least one hour. These walls shall be continuous from the floor slab to the underside of the floor or roof slab above, through any conccaled spaces such as those above the suspended ceilings and through interstitial structural and mechanical spaces. Doors with a 20-minute fire protection rating shall be used on openings other than those serving exits or hazardous areas. Doors shall be provided with latehes of a type suitable for keeping the door tightly closed and aceeptable to the authority having jurisdietion. Transfer grills, whether protected by fusible link-operated dampers or not, shall not be used in these walls or doors. Fixed wired glass vision panels may be placed in corridor walls, provided thcy do not exceed 1,296 square inches in size and are installed in approved steel frames. Fixcd wired glass vision panels may be installcd in wood doors, provided they do not exceed 720 square inches in size and are installed in approved steel frames.

Exception No. 1: In institutional occupancies equipped throughout with an approved automatic extinguishing system, corridors may be separated from use areas by partitions. Doors in such partitions may be solid bonded wood core $11 / 4$ inches thick, or the equivalent. Doors shall be equipped with latches for kecping the doors tightly closed and may bc provided with glass vision panels without restriction.

$$
\text { C-11 }
$$




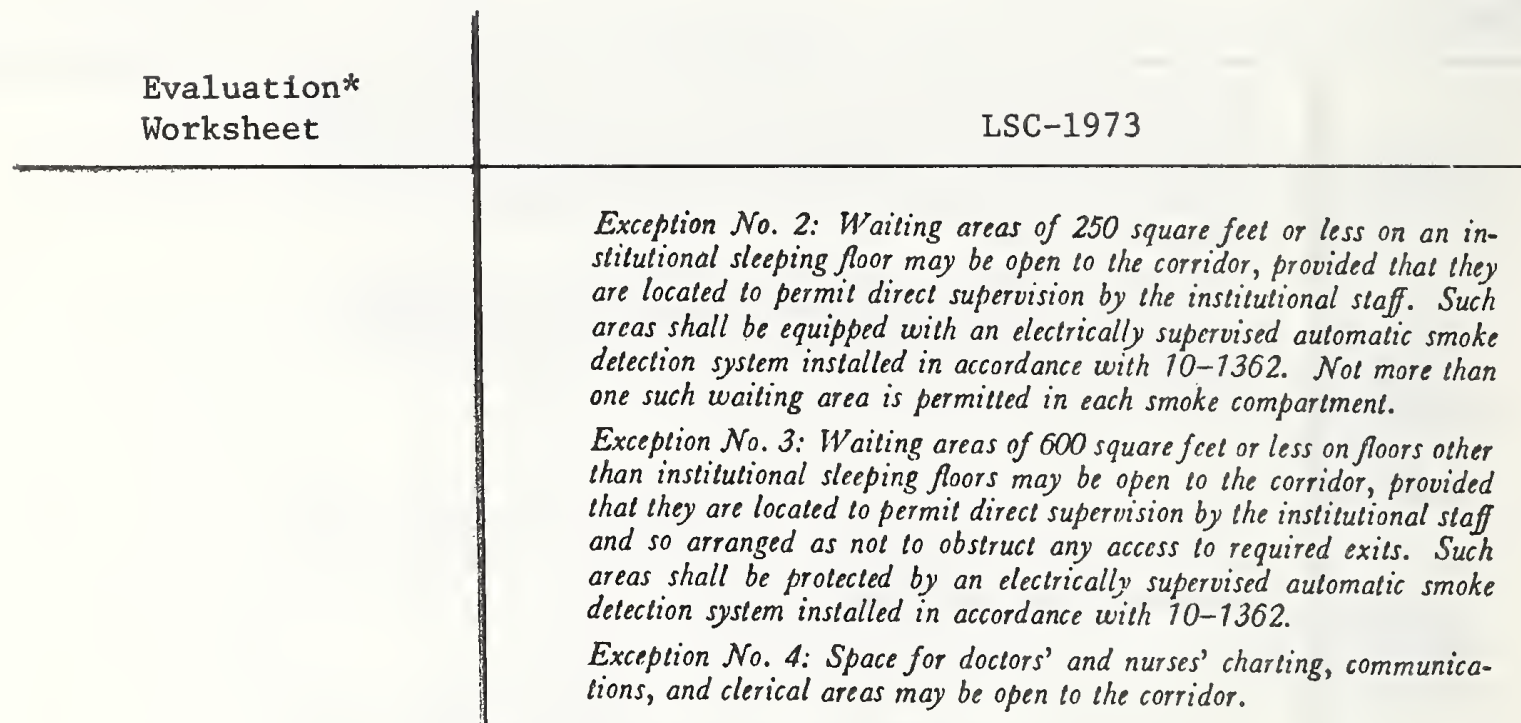

7.

7.

$2 .-3$.

$2 .-3$.

\section{0-134. Protection of Vertical Openings and Firestopping}

10-1341. Any stairway, ramp, elevator shaft, light and ventilation shaft, chute and other openings between stories shall be enclosed with noncombustible materials and in accordance with 6-1111, 6-1113, 6:1114, and 10-1323. A door in a stairway enclosure shall be self-closing, shall normally be kept in closed position and shall be marked in accordance with 5-2133.

10-1342. Firestopping shall be provided in accordance with 6-1311.

\section{0-135. Interior Finish}

10-1351. Interior finish of walls and ccilings in means of egress and of any room shall be Class A in accordance with Section 6-2.

Exception No. 1: Walls and ceilings may be of Class $B$ materials in individual rooms of not over four persons in capacity.

Exception No. 2: The provisions of Section 6-2, permitting a class of interior finish with a higher flamespread in buildings with automatic sprinklers, shall not apply for institutional occupancies.

10-1352. Floor finish material shall be Class A or B throughout all hospitals, nursing homes and residential-custodial care facilities.

Exception No. 1: The provisions of Section 6-2, permitting a class of interior finish with a higher flamespread in buildings with automatic sprinklers, shall not apply 10 foor finish material in institutional occupancies.

Exception No. 2: Tongue and groove wood flooring, one-half inch thick or greater, shall be permitted in institutional buildings equipped throughout with an approved automatic cxtinguishing sysiem.

$$
\mathrm{C}-12
$$


10-1361*. Every building shall have an electrically supervised, manually operated fire alarm system, in accordance with Section 6-3. The firc alarm system shall be installed to transmit an alarm automatically to the fire department that is legally committed to serve the area in which the institution is located, by the most direct and reliable method approved by local regulations. Internal audible alarm devices shall be provided in accordance with Section 6-3.

Exception: Prc-signal systems shall not be permitted in institutional occupancies.

12 .

10-1362. An approved automatic smoke detection system shall be installed in all corridors of hospitals, nursing homes, and residentialcustodial care facilities. Such systerns shall be installed in accordance with the applicable standards listed in Appendix B, but in no case shall smoke detectors be spaced further apart than 30 feet on centers or more than 15 feet from any wall. All automatic smoke detection systems required by this section shall be electrically interconnected to the fire alarm system.

Exceplion: Where each patient sleeping room is prolected by such an approved detection system and a local delector is provided at the smoke partition, such corridor systems will not be required on the patient sleeping room floors.

12.

10-1363. Required fire detection devices or systems shall be in accordance with Section $6-3$ and shall be electrically interconnected to the manually operated fire alarm system.

13.

10-1364*. Automatic fire extinguishing protection shall be provided throughout all hospitals, mursing homes, and residential-custodial care facilities. (See 10-132 for construction lypes permilled.)

Exception: Buildings of firc-resistive or one-story protected noncombustible construction.

13.

10-1365. Where exceptions are stated in the provisions of this Code for institutional occupancies equipped throughout with an approved automatic extinguishing systcm, such systems shall be in complete accordance with the requirements of 10-1366.

13.

10-1366. Required automatic sprinkler systems shall be in accordance with Section 6-4 for systems in light hazard occupancies and shall be electrically interconnected with the fire alarn system. The main sprinkler control valve shall be electrically supervised so that at least a local alarm will sound when the valve is closed. 


\begin{tabular}{l|l}
$\begin{array}{l}\text { Evaluation* } \\
\text { Worksheet }\end{array}$ & \multicolumn{1}{|c}{ LSC-1973 } \\
\hline 8. & $\begin{array}{l}10-1367 . \text { The sprinkler piping, serving no more than } 6 \text { sprinklers } \\
\text { for any isolated hazardous area. may be connected directly to a } \\
\text { domestic water supply system having a capacity sufficient to provide } \\
0.15 \text { gallons per minute per square foot of foor area throughout the } \\
\text { ientire enclosed area. An outside-screw-and-yoke shutof valve shall } \\
\text { be installed in an accessible location between the sprinklers and } \\
\text { the connection to the domestic water supply. }\end{array}$ \\
\hline
\end{tabular}

7. -8 .

10-1368. Sprinkler requirements for hazardous areas are stated in 10-1371 and sprinkler requirements for chutes are given in 7-1131.

I.

10-1369. Portablc fire extinguishers shall be provided in all institutional occupancies in accordance with Section 6-4.

\section{0-137. Hazardous Areas}

10-1371.* Any hazardous area shall bc safeguarded in accordance with Section 6-5. Hazardous areas include, but are not restricted to the following. Those arcas accompanicd by a dagger $(t)$ in the list shall have both separation and a complete extinguishment system.

Boilcr and heater rooms

Laundries

Kitchens

Repair shops

Handicraft shops

Employce locker rooms

†Soiled linen rooms

†Paint shops

†Rooms or spaces, including repair shops, used for the storage of conbustible supplies and equipment in quantities deemed hazardous by the authority having jurisdiction.

†Trash collection rooms

Gift shops

8.

10-1372. Laboratorics shall be protected in accordance with the applicable standard listed in Appendix B.

10-14. Building Service Equipment

10-141. Air-Conditioning, Ventilating, Heating, Cooking, and Other Service Equipment

10-1411. Air-conditioning, ventilating, heating, cooking, and other service equipment shall be in accordance with Chapter 7 , and shall be installed in accordance with the manufacturer's specifications.

Exception: As modified in 10-1412 and 10-1413 below.

$$
\mathrm{C}-14
$$


D.

C.

7.

N.A.

N.A.
10-1412.* Portable comfort heating devices are prohibited. Any heating device other than a central heating plant shall be so designed and installed that combustible material will not be ignited by it or its appurtenances. If fuel fired, such heating devices shall be chimney or vent connected, shall take air for combustion directly from outside, and shall be so designed and installed to provide for complete separation of the combustion system from the atmosphere of the occupied arca. The heating system shall have safety devices to immediately stop the flow of fuel and shut down the equipment in case of either excessive temperatures or ignition failure.

Exception No. 1: Approved suspended unil heaters may be used, except in means of egress and patient slecping areas, provided such heaters are located high enough to be out of the reach of persons using the area and provided they are equipped with the safety devices called for above.

Exception No. 2: Fireplaces may be installed and used only in areas other than patient sleeping areas, provided that these areas are separated from patient sleeping spaces by construction having a 1-hour fire resistance rating and they comply with the appropriate standard listed in Appendix $B$. In addition thercto, the fireplace shall be equipped with a hearth that shall be raised at least 4 inches, and a heat tempered glass fireplace enclosure guaranteed against breakage up to a temperalure of $650^{\circ}$ Fahrenheit. If, in the opinion of the authority having jurisdiction, special hazards are present, a lock on the enclosure and other safety precautions may be required.

10-1413. Combustion and ventilation air for boiler, incinerator or heater rooms shall be taken directly from and discharged directly to the outside air.

10-1414. Any rubbish chute and linen chute including pneumatic systems shall be safeguarded in accordance with 7-113. An incinerator shall not be directly flue-fed nor shall any foor charging chute directly connect with the combustion chamber. Any trash chute shall discharge into a trash collecting room used for no other purpose and protected in accordance with Section 6-5.

\section{0-15. Windowless Buildings}

10-1511. See Section 16-4 for requirements for windowless buildings.

\section{0-2. EXISTING HOSPITALS, NURSING HOMES, AND RESIDENTIALCUSTODIAL CARE OCCUPANCIES}

10-211. Application

10-2111. This section establishes life safety requirements for all existing hospitals, nursing homes, and residential-custodial care institutions. Where requirements vary, the specific occupancy, such as hospital, nursing home, nursery, residential-custodial care institution, home for the aged, or mentally retarded care institution is named in the paragraph pertaining thereto. See Chapter 17 for operating fratures.

C-15 


\begin{tabular}{l|l}
$\begin{array}{l}\text { Evaluation* } \\
\text { Worksheet }\end{array}$ & \multicolumn{1}{|c|}{ LSC-1973 } \\
\hline N.A. & $\begin{array}{l}\text { 10-212. Modification of Retroactive Provisions } \\
\text { 10-2121. The requirements of this section may be modified if their } \\
\text { application would be clearly impractical in the judgment of the } \\
\text { authority having jurisdiction and if the resulting arrangement could } \\
\text { be considered as presenting minimum hazard to the life safety of the } \\
\text { occupants. The requirements may be modified by the authority } \\
\text { having jurisdiction to allow alternative arrangements that will } \\
\text { secure as nearly equivalent safety to life from fire as practical; but } \\
\text { in no case shall the modification afford less safety than compliance } \\
\text { with the corresponding provisions contained in the following part of } \\
\text { this Code. }\end{array}$
\end{tabular}

N.A.

10-2122.* A limited but reasonable time shall be allowed for compliance with any part of this section, commensurate with the magnitude of expenditure and the disruption of services.

N.A.

10-2123. When alternate protection is installed and accepted, the institution shall be considered as conforming for purposes. of this Code.

1.

\section{0-213. Conversions, Additions, and Modernization}

10-2131. No existing building shall be converted to a hospital, nursing home, or residential-custodial care institution unless it complies with all requirements for new institutional buildings.

1.

10-2132. A new addition to an existing institution shall be in conformance with Section 10-1 of this Code. The new addition shall be separated from the existing institution by noncombustible construction having a fire resistance rating of at least 2 hours, unless the existing institution conforms to the requirements of Section $10-1$ of this Code.

1.

10-2133. No construction in either modernization or renovation projects shall diminish the fire safety features of the institution currently in effect. Alterations or installations of new building services equipment shall be accomplished as nearly as possible in conformance with the requirements for new construction.

1.

\section{0-214. Occupancy and Occupant Load}

10-2141. Institutional occupancies in buildings housing other occupancies shall be completely separated from them by noncombustible construction having a fire resistance rating of at least two hours. 
1.

10.

10.

10.

10.

10 .

20.
10-2142.* Sections of institutional buildings may come under other occupancy classifications if they meet all of the following conditions:

(a) They are not intended to serve institutional occupants for purposes of housing, treatment, customary access, or means of egress.

(b) They are adequately separated from areas of institutional oc* cupancies by construction having a fire resistance rating of at least two hot:s.

10-2143. Auditoriums, chapels, residential areas, garages, or other occupancies in connection with hospitals or nursing homes shall have exits provided in acrurdance with the other applisable sections of this Cude.

10-2144. The occupant load for which means of egress shall be provided for any floor shall be the maximum number of persons intended to ocrupy that floor, but not less than 1 persnn for each 120 square fect gross floor area in institutional sleeping departments and not less than 1 person for each 240 square feet of gross floor area of inpatient institutional treatment departments. Gross floor areas shall be measured within the exterior building walls with no deductions.

\section{0-22. Exit Details}

\section{0-221. Number and Types}

10-2211. Exits shall be restricted to the following perinissible types:

(a) Doors leading directly outside the building (see Section 5-2)

(b) Interior stairs and smokeproof towers (see 10-225)

(c) Horizontal exits (see 10-226)

(d) Ramps (see 10-2252)

(e) Outside stairs (see Section 5-4)

(f) Exit passageways (see Section 5-7)

10-2212. At least 2 exits of the above types, remote from each other, shall be provided for each floor or fire section of the building. At least 1 exit in each floor or fire section shall be as indicated in 10-2211 (a), (b), (c), or ( $)$.

10-2213. Revolving doors shall not be counted as required exits, and shall not be installed except as specifically stated in Section 5-2.

10-2214. Elevators constitute a supplementary facility, but are not counted as required exits. 
10.

10.

6. -10

\section{0-222. Capacity of Exits}

10-2221. The capacity of any required exit shall be based on its width in units of 22 inches as defined in 5-115. The capacity of (a) exits providing travel by means of stairs shall be 22 persons per exit unit, and (b) exits providing travel without stairs, such as doors or horizontal exits, shall be 30 persons per exit unit.

Exception: The capacity of exits in Institutional Occupancies equipped throughout with an approved automatic fire extinguishing system may be increased 1035 persons per exit unit for travel by means of stairs, and to 45 persons per exit unit for travel without stairs.

\section{0-223. Access to Exits}

10-2231. Every aisle, passageway, corridor, exit discharge, exit Lcation and access shall be in arcordance with Section 5.1, exccpt as modified bclow.

10-2232. Travel distance (a) between any room door intended as exit access and an exit shall not exceed 100 feet; (b) between any point in a room and an exit shall not exceed 150 feet; (c) between any point in an institutional sleeping room or suite and an exit access door of that room or suite shall not exceed 50 feet. Travel distance shall be measured in accordance with 5-119.

Exception: The travel distance in $(a)$ or $(b)$ above may be increased by 50 feet in buildings completely equipped with an automatic fire extinguishing system.

10.

10-2233. Every institutional sleeping room, unless it has a door opening at ground level, shall have an exit door access leading directly to a corridor which leads to an exit. One adjacent room such as a sitting or anteroom may intervene if all doors along the path of exit travel are equipped with nonlockable hardware, except as provided in 10-2242, and this intervening room is not intended to serve more than 8 institutional sleeping beds.

10.

10-2234. Any required aisle, corridor, or ramp shall be not less than 48 inches in clear width when serving as means of egress from institutional sleeping rooms. It shall be of such width and so arranged as to avoid any obstructions to the convenient removal of nonambulatory persons carried on stretchers or on mattresses serving as stretchers.

10.

10-2235. Any room, and any suite of rooms, as permitted in 10-2233, of more than 1,000 square feet shall have at least 2 exit access doors remote froni each other.

$6 .-10$

10-2236. Every corridor shall provide access to at least two approved means of egress from the building in accordance with 5-120, without passing through any intervening rooms or spaces other than corridors or lobbies. Existing dead-end corridors are undesirable and shall be altered wherever possible so that exits will be accessible in at least 2 different directions from all points in aisles, passageways, and corridors. 
10.

\section{5. -10}

10.

9. -10

$7 \cdot-8 .-10$.

10.

10.
10-224. Doors

10-2241. Every door shall be in accordance with Section 5-2 except as modified below. For doors in horizontal exils and smoke partitions see 10-2261 and 10-231.

10-2242. Locks installed on institutional sleeping room doors shall be so arranged that thcy can bc locked only from the corridor side.

All such locks shall be arranged to permit-exit from the room by a simple operation without the use of a kcy.

Exception No. 1: Doors leading directly to the outside of the building may be subject to locking from the room side.

Exception No. 2: Doors in homes for the aged may be lockable by the occupant, if they can be unlocked from the opposite side and keys are carried by altendants at all times.

Exception No. 3: Locks permitted by 10-0004.

10-2243. Exit access doors to hospital and nursing home sleeping rooms, diagnostic and treatment areas such as, X-ray, surgery, and physical therapy, all doors between these spaces and the required exits, and all exit doors serving these, spaces shall be at lcast 42 inches wide. Doors to residential-custodial sleeping rooms and all exit doors serving these spaces shall be at least 32 inches wide.

Exception No. 7: Doors which are so located as not to be subject to use by an institutional occupant shall be not less than 28 inches in width as defined in 5-2141.

Exception No. 2: Doors in exit stairway enclosures shall be not less than 36 inches wide.

10-2244. Any door in a fire separation, horizontal exit or a smoke partition may be held open only by an electrical device which complies with 5-2134. The device shall be so arranged that the operation of the following will initiate the self-closing action:

(a) The manual alarm system required in 10-235 and either b or c below.

(b) A local smoke detector designed to detect smoke passing through the opening.

(c) A complete and approved automatic fire extinguishing system or automatic fire detection system.

10-2245. Doors in stair enclosurcs or in walls separating hazardous areas shall not be equipped with hold-open devices.

\section{0-225. Stairs, Smokeproof Towers, Ramps}

10-2251. Every stair and smokeproof tower shall be in accordance with Section 5-3 and shall be Class A or B.

Exception: Any cxisting interior stair not complying with Section 5-3 may be continued in use subject to the approval of the authority having jurisdiction.

10-2252. Every ramp shall be in accordance with Section 5-6, and shall bc Class $\mathrm{A}$ or Class $\mathrm{B}$. Ramp width shall be as specified in 10-2234.

$$
\text { C-19 }
$$




\begin{tabular}{|c|c|}
\hline $\begin{array}{l}\text { Evaluation* } \\
\text { Worksheet }\end{array}$ & LSC-1973 \\
\hline 10. & 10-226. Horizontal Exits \\
\hline & $\begin{array}{l}\text { 10-2261.* A door in a horizontal exit shall be at least } 42 \text { inches } \\
\text { wide and shall be in accordance with Section } 5-5 \text {, except as modi- } \\
\text { fied herein. At least } 30 \text { net square feet per institutional occupant } \\
\text { shall be provided for the total number of institutional occupants in } \\
\text { adjoining compartments. A door in a horizontal exit is not re- } \\
\text { quired to swing with exit travel as specified in } 5-5143 \text {. }\end{array}$ \\
\hline
\end{tabular}

B.

J.

9.

9.

9.

9.

9.

\section{0-227. Exit Lighting and Signs}

10-2271. Each hospital, nursing home and residential-custodiai care facility shall bc provided with emergency lighting in accordance with 5-10.

\section{Exception: Emergency lighting of at least one hour duration shall be} provided.

10-2272. Exit signs shall be provided in each nursing home and rcsidential-custodial care facility in accordance with Section 5-11.

Exception: Signs may be omitted in one-story buildings with an occupancy of less than 30 persons.

\section{0-23. Protection}

\section{0-231. Subdivision of Building Spaces}

10-2311. Smoke partitions shall be provided, regardlcss of building construction, as follows:

(a) To divide every story used for sleeping rooms for more than 30 institutional occupants into at least two compartments, and

(b) To limit on any story the maximum area of each smoke compartment to no more than 22,500 square feet of which both length and width are limited to $150 \mathrm{ft}$.

Exception: Protection may be accomplished in conjunction with the provision of hcrizontal exits.

10-2312. Smoke partitions shall be constructed in accordance with Scction 6-6 and shall have a fire resistance rating of at least one-half h:sur.

10-2313. Smokc partitions shall be provided on stories which are usable but unoccupied.

10-2314. Space shall be provided on both sides of the smoke partition(s) or in each arca of refuge for the total number of institutional occupants scrved.

10-2315. Opcnings in smokc partitions shall bc protected by wired glass panels in steel frames or by $13 / 4$ inch solid bonded wood core doors as a minirnum. 
9.

1.

1.

1.

1.

1.

4.

4.

10-2316. Doors in smoke partitions shall be self-closing or kept in the open position provided they meet the requirement of 10-2244. Such doors shall not be required to swing with exit travel.

\section{0-232.* Minimum Construction Standards for Existing In-} stitutions.

10-2321. For the purpose of this section, stories shall be counted starting at the lowest floor of exit discharge.

10-2322. Institutional buildings of one story in height only may be of any type of construction (see 10-2352 for extinguishntent requirements).

10-2323. Institutional buildings up to and including two stories in height may be constructed of fire resistive construction protected noncombustible construction, protected ordinary construction, protected wood frame construction, heavy timber construction, or unprotected noncombustible construction. (See 10-2352 for automalic extinguishment requirements.)

10-2324. Institutional buildings three stories, or more, in height shall be of fire resistive construction.

Exception: Institutional buildings up 10 and including three stories in height may be of protected noncombustible construction if equipped throughout with an automatic extinguishing system.

10-2325. Every interior wall and partition in buildings of fireresistive and noncombustible construction shall be of non-com bustible materials.

10-2326. Every institutional sleeping room shall have an outside window or outside door arranged and located to permit the venting of products of combustion and to permit any occupant to have access to fresh air in case of emergency. (See 10-0004 for detention screen requirements.)

Exceplion: Rooms housing obstetrical labor beds, recovery, emergency observation beds, and newborn bassinets.

10-2327. Corridors in existing institutional occupancies shall be separated from use areas by walls constructed to resist the passage of smoke. Doors in such corridor partitions, other than those serving exits or hazardous areas, shall be at least $13 / 4$ inch solid bonded wood core or equivalent. Doors shall be provided with latches of a type suitable for keeping the door tightly closed and acceptable to the authority having jurisdiction.

10-2328. Transonis, louvers, or transfer grills, whether protected by fusible link-operated dampers or not, shall be closed and made smoke tight by permanent noncombustible construction.

$$
\text { C-21 }
$$




\begin{tabular}{|c|c|}
\hline $\begin{array}{l}\text { Evaluation* } \\
\text { Worksheet }\end{array}$ & LSC-1973 \\
\hline 4. & $\begin{array}{l}\text { 10-2329. Glass vision panels in such corridor walls or doors shal } \\
\text { be fixed wired glass in approved stecl frames, limited to } 1296 \text { sq. in } \\
\text { per pancl. } \\
\text { Exception: Institutional occupancies equipped throughout with an ap- } \\
\text { proved automatic extinguishing system, may be provided with g'ass } \\
\text { vision panels without restriction. }\end{array}$ \\
\hline
\end{tabular}

7.

10-233. Protection of Vertical Openings and Firestopping.

10-2331. Each stairway between stories shall be enclosed in accordance with 6-1113 and 6-1114 with partitions having a 1-hour fire resistance rating.

Exception No. 1: Where a full enclosure is impracticable and the stair is not a required cxit, the required enclosure may be limited to that necessary to prevent a fire originating in any story from sprcading to any ocher story.

Exception No. 2: Stairs that do not connect 10 a corridor, do not connect more than two levels, and do not serve as a means of egress need not comply with these regulations.

7.

10-2332. Any elevator,shaft, light and ventilation shaft, chute, and other vertical opening between stories shall be protected as required above for stairways.

7 .

10-2333. Each exterior wall of frame construction and interior stud partitions shall be firestopped so as to cut off all concealed draft openings, both horizontal and vertical, between any cellar or basement and the first floor. Such firestopping shall consist of suitable noncombustible material or of wood at least 2 inches (nominal) thick.

7.

10-2334. Any existing linen and trash chute which opens directly on to any corridor shall be sealed by fire-resistive construction to prevent further use or shall be provided with a fire door assembly suitable for a Class $B$ location and having a fire protection rating of $11 / 2$ hours. All new chutes shall comply with 7-113.

\section{0-234. Interior Finish}

$2 .-3$.

10-2341.* Interior finish shall be Class $A$ or Class $B$ in accordance with Section $6-2$. In buildings equipped with a complete automatic firc extinguishing systern, Class $C$ interior finish may be continued in usc, except in means of egress. 
10-2351. Every building shall have a manually operated firealarm system, in accordance with Section 6-3. Audible alarm devices shall be used.

Exception No. 1: Presignal systems shall not be permitted in institutional occupancies.

Exception No. 2: Where visual alarm devices have been installed in patient sleeping areas, they may be accepted by the authority having jurisdiction. vided throughout all hospitals, nursing homes, and residentialcustodial care facilities.

Exception: Buildings of fire-resistive construction of any height or protected noncombustible conslruction not over 1 story in height.

10-2353. Any required automatic sprinkler system shall be in accordance with Section 6-4, for systems in light hazard occupancies, and shall be electrically interconnected with the fire alarm system. The main sprinkler control valve shall be electrically supervised so that at least a local alarm will sound when the valve is closed.

10-2354. The sprinkler piping, serving no more than six sprinklers

8. for any isolated hazardous area, may be connected directly to a domestic water supply system having a capacity sufficient to provide 0.15 gallons per minute per square foot of floor area throughout the entire enclosed area. An outside screw-and-yoke shutoff valve shall be instailed in an accessible location between the sprinklers and the connection to the domestic water supply.

I.

10-2355. Portable fire extinguishers shall be provided in all institutional occupancies in accordance with 6-422.

\section{U-236 Hazardous Areas}

8.

10-2361. Any hazardous area shall be safeguarded in accordance with Section 6-5. Hazardous areas include, but are not restricted to the following:

Boiler and hcater rooms

Laundries

Kitchens

Repair shops

Handicraft shops

Employee locker rooms

Soiled linen rooms
Rooms or spaces used for storage of combustible supplies and equipment in quantities deemed hazardous by the authority having jurisdiction

Trash collection rooms Gift shops

8.

10-2362. Laboratories shall be in accordance with the applicable standard listed in Appendix B. 


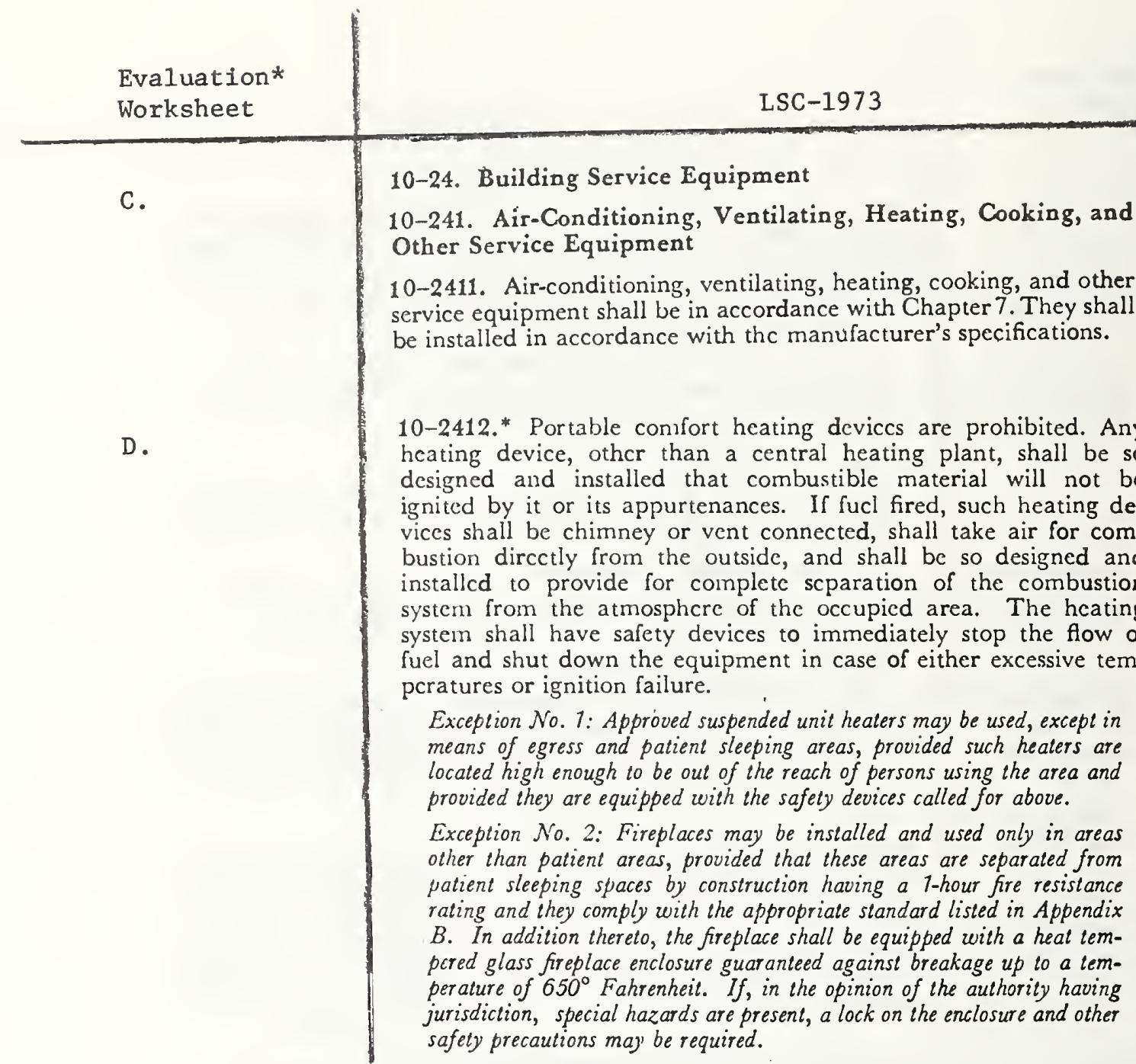

c.

10-2413. Combustion and ventilation air for boiler, incinerator, or heater rooms shall be taken directly from and discharged directly to the outside air.

10-2414. Any rubbish chute and linen chute including pneumatic systems shall be safeguarded in accordance with 7-113. Existing fue-fed incinerators shall be sealed by fire-resistive construction to prevent further use. Any trash chute shall discharge into a trash collecting room used for no other purpose and protected in accordance with Section 6-5.

N.A.

10-25. Windowless Buildings

10-2511. Sce section 16-4 for requirements for windowless buildings. 


\section{7-11. Firc Exit Drills}

F.

17-1111.* Fire exit drills conforming to the provisions of this Chapter of the Code shall be regularly conducted in schools through grade 12 and in other occupancies where specified by the provisions of Chapters 8 through 16 , or by appropriate action of the enforcing authority having jurisdiction, but with any necessary modifications in detail of procedures to make the drills most effective for their intended purpose in any individual building.

F.

17-1112. Fire exit drills, where required, shall be held with sufficient frequency to familiarize all occupants with the drill procedure and to have the conduct of the drill a matter of established routine.

F.

17-1113.* Drills shall be held at unexpected times and under varying conditions to simulate the unusual conditions obtaining in case of fire.

F.

17-1114. Responsibility for the planning and conduct of drills shall be assigned only to competent persons qualified to exercise leadership.

F.

17-1115. In the conduct of drills emphasis shall be placed upon orderly evacuation under proper discipline rather than upon speed as such; no running or horseplay shall be permitted.

F.

F.

17-1116.* Drills shall include suitable procedures to make sure that all persons in the building, or all persons subject to the drill, actually participate.

17-1117. Fire alarm facilities, where available, shall be regularly used in the conduct of fire exit drills.

$$
\text { H. }
$$

\section{7-12. Furnishings and Decorations}

17-1211. No furnishings, decorations, or other objects shall be so placed as to obstruct exits, access thereto, egress therefrom, or visibility thereof. 


\begin{tabular}{|c|c|}
\hline $\begin{array}{l}\text { Evaluation* } \\
\text { Worksheet }\end{array}$ & LSC-1973 \\
\hline $\mathrm{H}$. & $\begin{array}{l}\text { 17-1212.* Flammable furnishings or decorations shall be flame } \\
\text { retardant where required by the applicable provisions of this } \\
\text { Chapter. }\end{array}$ \\
\hline н. & $\begin{array}{l}\text { 17-1213.* No furnishings or decorations of an explosive or highly } \\
\text { flammable character shall be used in any place of assembly or } \\
\text { other occupancy. } \\
\text { Exception: Privale dwellings. }\end{array}$ \\
\hline 13. & $\begin{array}{l}\text { 17-13. Automatic Sprinkler Systems } \\
\text { 17-1311. All automatic sprinkler systems required by this Code } \\
\text { shall be continuously maintained in reliable operating condition } \\
\text { at all times, and such periodic inspections and tests shall be made } \\
\text { as are necessary to assure proper maintenance. }\end{array}$ \\
\hline
\end{tabular}

11.

\section{7-14. Alarm and Fire Detection Systems}

17-1411. Systems shall be under the supervision of a responsible person who shall cause proper tests to be made at specified intervals and have general charge of all alterations and additions.

11. -12 .

11. -12 .

N.A.

\section{7-15. Fire Retardant Paints}

17-1511. Fire retardant paints or solutions shall be renewed at such intervals as necessary to maintain the necessary flame re-
tardant properties.

\section{7-16. Recognition of Means of Egress}

17-1611. Hangings or draperies shall not be placed over exit doors or otherwise located as to conceal or obscure any exit. Mirrors shall not be placed on exit doors. Mirrors shall not be placed in or adjacent to any exit in such a manner as to confuse the direction of exit. 
F.

N.A.

F.

F.

F.

\section{7-411. Attendants, Evacuation Plan, Fire Exit Drills}

17-4111. The administration of every hospital, nursing home and residential-custodial care institution shall have in effect and available to all supervisory personnel written copies of a plan for the protection of all persons in the event of fire and for their evacuation to areas of refuge and from the building when necessary. All employees shall be instructed and kept informed respecting their duties under the plan. A copy of the plan shall be readily available at all times in the telephone operator's position or at the security center. The provisions of $17-4113$ to $17-4127$ inclusive shall apply.

17-4112. Every bed intended for use by institutional occupants shall be easily movable under conditions of evacuation and shall be equipped with the type and size casters to allow easy mobility, especially over elements of the structure such as expansion plates and elevator thresholds. The authority having jurisdiction may make exceptions in the equipping of beds intended for use in areas limited to patients such as convalescent, self-care, or psychiatric patients.

17-4113.* Fire exit drills in hospitals shall include the transmission of a fire alarm signal and simulation of emergency fire conditions except that the movement of infirm or bed-ridden patients to safe areas or to the exterior of the building is not required. Drills shall be conducted quarterly on each shift to familiarize hospital personnel (nurses, interns, maintenance engineers, and administrative staff) with signals and emergency action required under varied conditions. At least 12 drills shall be held every year. When drills are conducted between 9:00 P.M. and 6:00 A.M. a coded announcement may be used instead of audible alarms.

\section{7-412. Procedure in Case of Fire}

7-4121. Upon discovery of fire, personnel shall immediately take the following action:

(a) If any person is involved in the fire, the discoverer shall go to the aid of that person calling aloud an established code phrase. The use of a code provides for both the immediate aid of any endangered person and the transmission of an alarm. Any person in the area, upon hearing the code called aloud, shall transmit the interior olorm using the nearest manual alarm station.

(b) If a person is not involved in the fire, the discoverer shall transmit the interior alarm using the nearest manual alarm station.

(c) Personnel, upon hearing the alarm signal, shall immediately execute their duties as outlined in the institutional fire safety plan.

17-4122. The institutional telephone operator shall determine the location of the fire as indicated by the audible signal. In a building equipped with an uncoded alarm system, a person on the floor of fire origin shall be responsible for the prompt notification of the fire location to the institutional telephone operator.

$$
\text { C-27 }
$$




Evaluation*
Worksheet

F.

F.

F.

10.
17-4124. If the interior alarm system is out of service, any person discovering a fire shall immediately notify the telephone operator by telephone. The operator shall then transmit this to the fire department and alert the building.

17-4125. A written institutional fire safety plan shall provide for:
(a) Use of alarms
(b) Transmission of alarm to fire department
(c) Response to alarms
(d) Isolation of fire
(e) Evacuation of area
(f) Preparing building for evacuation
(g) Fire extinguishment.

17-4126. All institutional personnel shall be instructed in the use of, and response to, fire alarms; and, in addition, they should be instructed in the use of the code phrase to insure transmission of an alarm under the following conditions:

(a) When the discoverer of a fire must immediately go to the aid of an endangered person.

(b) During a malfunction of the interior alarm system.

Personnel hearing the code announced shall first transmit the interior alarm using the nearest manual alarm station and shall then immediately execute their duties as outlined in the institutional fire safety plan.

\section{7-413. Maintenance of Exits}

17-4131. Daily inspection and proper maintenance shall be provided to insure the dependability of the method of evacuation selected. Institutions which find it necessary to lock exits shall at all times maintain an adequate staff qualified to release and conduct occupants from the immediate danger area to a place of safety in case of fire or other emergency. Where patient room doors are locked, attendants shall carry keys to these doors. 
G.

H.

H.

H.

H.

\section{7-414. Smoking}

17-4141.* Smoking regulations shall be adopted and shall include the following minimal provisions:

(a) Smoking shall be prohibited in any room, ward, or compartment where flammable liquids, combustible gases, or oxygen are used or stored and in any other hazardous location. Such areas shall be posted with "NO SMOKING" signs.

(b) Smoking by patients classified as not responsible shall be prohibited.

Exception: When the patient is under direct supervision.

(c) Ashtrays of noncombustible material and safe design shall be provided in all areas where smoking is permitted.

(d) Metal containers with self-closing cover devices shall be readily available to all areas where smoking is permitted.

\section{7-415. Draperies}

17-4151.* Window draperies and curtains for decorative and acoustical purposes shall be flame retardant.

17-4152.* Cubicle curtains shall be noncombustible or rendered and maintained flame retardant.

\section{7-416. Furnishing and Decorations}

17-4161. Furnishings and decorations in institutional occupancies shall be in accordance with the provisions of 17-12.

17-4162.* Combustible decorations are prohibited in any institutional occupancy unless flame retardant. 



\section{INSTRUCTION MANUAL}

This manual is provided to assist in completion of the Fire/Smoke Zone Evaluation Work Sheet for Health Care Facilities. The step by step instructions for the mechanics of completing the work sheet are included in the work sheet itself. They are not repeated in this manual. This manual provides expanded discussion and definitions of the various items in the work sheet to assist the surveyor or reviewer when questions of definitions or interpretation arise. The manual is organized to progressively follow the format of the work sheet.

\section{CONTENTS}

\section{Fire/Smoke Zone}

Selection of Zones to be Evaluated Maintenance

Occupancy Risk (General Discussion)

Patient Mobility (Mobility Status)

Patient Density (Patient Factor)

Zone Location (Floor Factor)

Ratio of Patients to Attendants (Patients-Attendants Factor)

Page

Patient Average Age (Age Factor)

Safety Parameters (General Discussion)

Construction

Interior Finish (Corridor and Exit)

Interior Finish (Rooms)

Corridor Partitions/Walls

Doors to Corridor

$$
\begin{array}{r}
\text { D-2 } \\
\text { D-2 } \\
\text { D-3 } \\
\text { D-3 } \\
\text { D-3 } \\
\text { D-4 } \\
\text { D-4 } \\
\text { D-5 } \\
\text { D-5 } \\
\text { D-6 } \\
\text { D-6 } \\
\text { D-6 } \\
\text { D-7 } \\
\text { D-7 } \\
\text { D-8 } \\
\text { D-9 } \\
\text { D-9 } \\
\text { D-10 } \\
\text { D-11 } \\
\text { D-12 } \\
\text { D-13 } \\
\text { D-13 } \\
\text { D-14 } \\
\text { D-16 } \\
\text { D-19 } \\
\text { D-20 } \\
\text { D-21 }
\end{array}
$$

Zone Dimensions

Vertical Openings

Hazardous Areas

Smoke Control

Emergency Movement Routes

Manual Fire Alarm

Smoke Detection and Alarm

Automatic Sprinklers

Table D-2 Flame Spread Classification for Wall and Ceiling Finishes

Table D-3 Fire Resistive Partition Details

Table D-4 Hazardous Areas

NOTE: The paragraph references within this manual are to the 1973 Edition of the NFPA No. 101 Life Safety Code. The corresponding paragraphs for the 1976 Code are shown in parenthesis. 
Fire/Smoke Zone - A fire/smoke zone (zone) is a space which is separated from all other spaces by floors, horizontal exits, or smoke barriers. Where a floor is not subdivided by horizontal exits or smoke barriers the entire floor is the zone.

NOTE: Individual rooms exceeding 1,000 square feet of floor area shall be evaluated as follows:

a. If the room has a single exit, it shall be evaluated as a single dead-end zone.

b. If the room has 2 or more exits, it shall be evaluated as either a room in a zone or as a separate zone, whichever gives the better (higher) rating.

Selection of Zones to be Evaluated - For a complete evaluation, every zone in the health care facility should be evaluated individually. From a practical standpoint most health care facilities have repetitive arrangements so that a complete picture can be developed by evaluating typical zones until all combinations are evaluated. The zones selected should include:

A. Each type of patient zone having a different type of mobility, density, or attendant ratio as classified in Table 1 of the work sheet.

B. Each zone that represents a significantly different type of construction finish, or protection system.

C. Zones containing special medical treatment or support activities (operating suites, intensive care units, laboratories).

D. Zones not involving housing, treatment, or customary access for patients as follows:

1. Any zone, whether used for patient egress or not, may be evaluated on the same basis as a patient use zone. In such case the value of factor $F$ in Table 2 shall be assigned the value of factor L (Fire Zone Location) from Table 1. In such cases Item 10, Emergency Movement Routes shall be graded "Deficient Capacity" if the exit capacity is less than that prescribed for the actual occupancy of the space and as "<2 routes" if less than $75 \%$ of the prescribed exit capacity is present.

2. If the zone is separated from all patient use zones by two hour fire resistive construction (including any members that bear the load of a patient zone and with Class B fire doors on any communicating openings), it may be excluded from evaluation. In such case that space shall conform with the portion of the Life Safety Code appropriate to its use. In addition appropriate charges under Item 8, Hazardous Areas, in table 4 shall be charged against other zones in the facility. 
Maintenance - Any protection system, requirements, or arrangement which is not maintained in a dependable operating condition or is used in such a manner that the intended fire safety function or hazard constraint is impaired should be considered as defective and receive no credit in the evaluation.

Occupancy Risk (General Discussion) - In establishing a system for evaluating occupancy risk, it is recognized that: ( 1 ) there is a basic level of risk inherent in every health care facility; (2) that the fuel characteristics of furniture, equipment, and supplies vary with time; and (3) that the arrangement of these items within the space available also may vary with time.

Consequently, these three factors are not included as parameters in a safety equivalency measurement. To account for these factors, the occupancy risk base line is set at the inherent risk level with the presumption that the furniture, equipment and supplies will be the most combustible and adversely located (from a fire safety standpoint) of those normally found in health care facilities.

1. Patient Mobility

The single most important factor controlling risk in a health care facility is the degree to which patients must be assisted in taking actions necessary for their safety. The level of capability in health care facilities will vary from patients who, if informed or directed, will be able to take positive self-protecting actions to those patients who have no ability to move or even to take the simplest actions to safeguard themselves. In some cases patients may be directly connected to a fixed life support system and so intimately depended upon it that regardless of their physical condition or the availability of assistance they cannot be moved without jeopardy of death or serious harm. In the measurement of occupancy risk factors the least mobile category of patient expected in the zone determines the risk factor for that zone. The rationale for this approach is that if a zone accepts any patient with a reduced mobility status it may at any time increase the number of those patients. The impact of this approach will be that most health care facilities will be rated in the "not mobile" risk category.

Mobility Status - Patient mobility status is based on the capability of each patient to take actions necessary to protect himself. The four classes are defined as follows:

A. Mobile - Capable of readily rising from bed and taking self-protecting actions at approximately the same rate as a healthy adult. In order to be classified as mobile the patient must not require assistance in getting out of bed and must be able to open a closed or locked door. Mobile persons when sleeping shall be considered as mobile if they are not restrained or in any other. way reduced in response capabilities so that the type of arousal mechanism that would normally awaken an adult would not be affected. 
B. Limited Mobility - Those patients who have all of the capabilities of a mobile person except that their rate of travel will be significantly less.

C. Not Mobile - Incapable of removing themselves from danger exclusively by their own efforts. Examples would include persons who are totally bedridden, who require assistance to get out of bed or to move, or who are restrained, locked in their rooms, or otherwise prevented from taking complete emergency self protection evacuation actions without assistance. Mobility status should be based on the minimum level of mobility in an average 24 hour period.

D. Not Moveable - Not capable of being moved from the room in which they are housed through the course of a fire. Examples would include patients attached to life support systems or involved in medical or surgical procedures that prohibit their immediate relocation without extreme danger of death or serious harm.

\section{Patient Density}

The occupancy risk evaluation for occupancy density (number of patients within the zone) measures both the inherent increase in the maximum fire death potential that occurs as the number of patients in a zone increases, and the problems involved by a limited staff in handling larger numbers of patients during an emergency.

Patient Factor - The density of patients is the number of patients that could potentially be housed in the zone. The patient count should be based on the number of assignable beds in the zone on the assumption that they may all be occupied at the time of the fire emergency.

\section{Zone Location}

This risk factor relates to fire department accessibility to a fire. The rating system recognizes the inherent advantages of a first floor zone. It also recognizes the problems of evacuation from higher floors and the virtual impossibility of using external fire fighting efforts above the 6 th floor in any building.

Floor Factor - The measured zone's location shall be considered to be on floor 1 if the floor has direct access to the exterior at or within less than $1 / 2$ floor height above or below grade. If a building is on a sloping grade, each floor that has such exterior access shall be considered as a first floor situation for measurement of fire zones on those floors. The measured zone shall be considered on the second to third floor range, the fourth to sixth floor range, based on the height of the zone above the nearest grade floor. The zone shall be considered to be above the sixth floor if it is more than six floors above the nearest grade floor. The risk factor value for zones in basements is the same as for zones at or above the 7 th floor. The problems involved in emergency internal access, fire fighting and rescue, and the inability 
to make external attack in basements is approximately equivalent to that in upper stories of buildings.

\section{Ratio of Patients to Attendants}

This risk factor recognizes the importance of patient safety of a staff immediately available to respond in an emergency. The emergency actions that may be undertaken by the staff include detection, alarm, fire extinguishment, confinement of the fire, establishing barriers between the patients and the fire (closing patient room doors), rescue, emergency medical aid, and other related functions. A few of these functions, such as detection and alarming, may not be critically related to the ratio of nursing staff to patients while those ralated to rescue and the closing of patient room doors have a strong relationship to the staffing ratio. The staff ratio considered is based on the minimum staffing level immediately available (normally night hours).

Patients-Attendants Factor - The ratio of patients to attendants is based on those patients in the fire/smoke zone and the immediately available attendant staff. In calculating the ratio it shall be based on the minimum staffing level (usually occurring in the night shift). Where nursing stations or other positions of attendants are located at the junction of two or more zones and the location of the station is such that each of the zones has immediate access and view of the nursing station, then the total staffing assigned to the nursing station can be credited to each of the zones. An exception is where staff members are bound by duty assignments (cardiac care units, infant nurseries, operating suites, etc.) that prevent them from responding to other than their assigned zone.

The evaluation system assesses a charge of 4.0 to this risk factor in any case where there are periods when there are no attendants immediately available to a zone that houses patients.

5. Patient Average Age

This risk factor recognizes the increased susceptibility of the elderly and of infants up to one year of age to physical harm by smoke particles, gaseous combustion products and heated air. This rating assigns a larger risk factor to zones occupied by a population whose average age is above 65 or below one year. Basically, imposition of this charge demands additional safety protection in nursing homes for the aged and nurseries.

Age Factor - The mode value is used to arrive at the age factor for the patients in the zone. The calculation should be based on the past record of occupants assigned to the zone. Patients under one year old are classified at the same risk level as those over 65 . This is in recognition of the fire susceptibility of infants. 
Safety Parameters (General Discussion) - The safety parameters are a measure of those building factors that bear upon or contribute to the safety of those persons (patients, staff, visitors, others) who may be in the particular zone at the time of a fire.

Each of the safety parameters was analyzed. Where the current Code requirements recognize several different approaches to the parameter, the most important alternatives were listed. In addition, conditions likely to be encountered in situations failing to meet the explicit Code requirements, and conditions exceeding those required by the Code but available for increased protection were also listed.

\section{Construction}

Construction types are classified in accordance with the definitions of NFPA Standard No. 220 (1961 edition). Table D-1 is an abstract of the specific framing and material requirements for each of the classes of construction noted.

The requirements of NFPA Standard No. 220 for "interior partitions enclosing stairways or other openings through floors" are not to be considered in the construction classification. These floor openings and their protection are separately evaluated under safety parameter 7 , Vertical Openings.

Where the facility includes additions or connected structures of different construction the rating and classification of the structure shall be based on (a) separate buildings if a two hour or greater fire resistive separation exists between the portions of the building and on (b) the lower safety parameter point score involved if such a separation does not exist.

The floor level used to determine the parameter value is the floor of the fire zone being evaluated. The "Floor of Zone" is the story height above the floor of lowest exit discharge.

When the zone is on a floor below the floor of lowest discharge, the construction value shall be based on the distance of that floor from the closest level of discharge, i.e.: one floor below discharge = "Second"; two floors below discharge = "Third"; three or more floors below discharge = "Fourth and above."

\section{Interior Finish (Corridor and Exit)}

The classification of flame spread for corridor and exits is in accordance with the categories specified in the Life Safety Code and defined in Table D-2. The flame spread classification shall be based on the most combustible surface after deleting trim. No allowance is made in the Safety Parameter Values for Class D or E interior finishes. It is not anticipated that such material will be used in health care facilities. In the rare case such high flame spread interior finish material is 
involved an individual appraisal outside the capability of this evaluation system will be required.

\section{Interior Finish (Rooms)}

The same classification of interior finish applies to rooms as to corridors and exits. The specific definitions are in Table D-2. The flame spread classification shall be based on the most combustible surface after deleting trim. No consideration is included in the Safety Parameter Values for Class $D$ or $E$ interior finishes. It is not anticipated that such material will be used in health care facilities. In the rare case such high flame spread interior finish material is involved an individual appraisal outside of the capability of this evaluation system will be required.

\section{Corridor Partitions/Walls}

For the purpose of this evaluation, the fire resistive partitions considered are solely those between patient use areas and the corridors. A11 elements of the partition, except the door (considered as a separate element in this evaluation), must be included in the determination of its time-rated fire resistance classification, according to NFPA 251 (see Table D-3). An exception to the general rule of evaluating doors separate from walls occurs when one or more rooms has no door (see Safety Parameter 5). In this instance it is considered that the worth of the fire resistance capabilities of the corridor partition wall is so reduced that the wall should be graded as having no fire resistance. The mechanism for doing this is incorporated into the Fire Safety Evaluation Work Sheet.

Walls shall be considered as incomplete if they have unprotected openings (louvers, gaps, transfer grills) between the floor and the ceiling, or have ordinary glass lights.* If openings exist above the ceiling level (or even if the partitions stop at the ceiling level) the walls shall be considered as complete if the corridor ceiling is complete from wall to wall for the full extent of the fire zone. In such cases the ceiling and the corridor walls jointly perform the fire and smoke barrier functions normally expected of a corridor wall which extends from the floor slab to the underside of the floor or roof slabs above. In these instances any protection credited as corridor protection under Safety Parameter items 9, Smoke Contro1; 12, Smoke Detection and Alarm; or 13, Automatic Sprinklers must extend to all of the area covered by the ceiling involved.

*Ordinary glass lights shall not be considered as making a partition incomplete in locations where both sides of the glass light are fully protected by automatic sprinkler systems. 
Walls shall be considered to have less than a $1 / 3$ hour fire resistance rating if they are not equivalent to $1 / 2$ inch gypsum wall board on both sides of studs (even if they extend at least from floor to ceiling) or if they are not continuous above the ceiling to the underside of the floor or roof (or floor or roof assembly) above, through any concealed space such as above a suspended ceiling and through interstitial structure and mechanical spaces. Partitions shall also be rated as less than $1 / 3$ hour if they are not incomplete but other defects are involved or the criteria in Table $\mathrm{D}-3$ are not met.

Fire resistive partitions shall be considered as between $1 / 3$ and one hour if they meet all the criteria for continuity of construction and the criteria of Table $\mathrm{D}-3$ and have a fire resistance of between 20 minutes and one hour.

\section{Doors to Corridor}

The classification of doors to the corridor shall be based on the minimum quality of any door in the zone and the classification shall be determined in accordance with NFPA 252. Doors for protection of hazardous areas and stairwells are not included in this evaluation. They are covered separately in safety parameter items 7 and 8 .

A. No Door - A room shall be considered as not having a door if there is no door in the opening or if there is some other mechanism which prevents closing of the door or otherwise leaves a significant opening between the patient room and the corridor. Doors with louvers or ordinary glass lights* shall be classified as "no door". Doors which have been blocked open by door stops, chocks, tie backs or other devices which require manual unlatching or releasing action to close the door shall be classified as "no door". Also doors that are not provided with a latch suitable for keeping the door tightly closed shall be classified as "no door".

B. Door Less than 20 Minutes of Fire Resistance - Doors which are not deficient as described in $A$, but which do not meet the requirements for $C$ below, will be classified as less than 20 minutes of fire resistance.

C. Door 20 Minutes or More Fire Resistance - Doors shall be considered as having 20 minutes or greater fire resistance if they are of $13 / 4$ " thick solid core wood construction or any other arrangement of equal or greater stability and fire integrity. The thermal insulation capability of the door is not considered. Hollow or sheet steel doors therefore meet the 20-minute requirement.

*Ordinary glass lights shall not be considered as making a partition incomplete in locations where both sides of the glass light are fully protected by automatic sprinkler systems. 
D. 20 Minutes or More Fire Resistance and Automatic Closing - Automatic closing devices shall be considered present if the door has an arrangement which holds them open in a manner such that they will be released by a smoke detector operated device (e.g. magnetic or pneumatic hold open device) prior to the passage of significant smoke from a room of fire origin into the corridor or from the corridor into a room not involved in the fire. The requirement for 20 minutes of fire resistance is the same as in $\mathrm{C}$ above.

Self-Closing Patient Room Doors - Traditional self-closing doors on individual patient rooms shall be evaluated in the following manner:

1. If it can be established that the doors are constantly kept in the normally closed position except when persons are actually passing through the openings, the self-closing device shall be considered as equal to an automatic closing device and credited accordingly.

2. If the self-closing doors are blocked open they shall be classified as "no door" and a charge of (-10) invoked.

\section{Zone Dimensions}

Zone length is measured as the length of the corridor in the smoke zone.

The length of a corridor "dead end" shall be measured from the point at which a person egressing from the dead end would have an option of egressing in two separate directions.

In assessing the values for this parameter, a single value will be chosen based on the poorest safety level in the zone. For example, if one or more dead ends in excess of 30 feet exist, the charge for dead ends $(-4)$ shall be applied regardless of the actual corridor lengths.

Since dead end corridors and single emergency movement routes (covered in item 10, Emergency Movement Routes) will each confine the occupants of a fire zone to a single means of egress, the charges for these two items are not cumulative. As indicated by the footnote on the safety parameter values page in the Fire Safety Evaluation Work Sheet, the charge for dead end corridors is to be a value of 0 instead of either $(-4)$ or $(-6)$ in the special case where a charge of $(-8)$ is assessed under item 10 for single emergency movement routes.

\section{Vertical Openings}

These values apply to vertical openings and penetrations including exit stairways, ramps and other vertical exits of the type recognized by the Life Safety Code, pipe shafts, ventilation shafts, duct penetrations, and laundry and incinerator chutes. Enclosures shall be of construction having fire resistance not less than that prescribed for vertical openings (see safety parameter item 7). In addition, they shall be equipped with D-9 
fire doors or acceptable protection of openings into the shafts also designed and installed as to provide a complete barrier to the vertical spread of fire or smoke. A stairway shall be considered open if it is: (a) unenclosed; (b) is enclosed but does not have doors; (c) is enclosed but has openings other than doorways; and (d) is enclosed and has doors but the doors do not have self-closers.

Where vertical openings are located outside the fire/smoke zone and the separation between the zone and vertical opening is of one-hour or greater fire resistance and is of higher fire resistance than the protection of the vertical opening itself, (for example: an open shaft separated from the zone by a two-hour fire resistive partition with Class B self-closing fire doors) the rating of this factor for the zone being measured shall be based on the higher of the two fire resistant categories. In the above example, a safety parameter value of 3 would be given for the two-hour fire resistance. When this occurs however the space with the vertical opening cannot be considered as an exit route or refuge area for that zone when considering safety parameter 10, Emergency Movement Routes.

A vertical opening shall be considered as open for greater than three floors if there is unprotected penetration of four or more floors on the same shaft without an intervening slab or other cutoff. (Also see same area as an unprotected penetration covered in the discussion of item 13, Automatic Sprinklers.)

\section{Hazardous Areas}

Hazardous area protection is determined in accordance with Section 6-5 of the 1973 Life Safety Code. In assessing the charge for hazardous areas only one charge shall be made. It shall be the most severe charge corresponding to the deficiencies present. A double deficiency can exist only where the hazard is severe and the space is not sprinkler protected. Double protection consists of both a fire resistive enclosure and automatic sprinkler protection of the hazardous area. If both of these are lacking for a severe hazardous location, the double deficiency charge shall be made. If double deficiencies exist both in the zone and outside the zone, the higher charge (-11) for the condition inside the zone shall be made. The charges are not cumulative regardless of how many hazardous areas are present.

Where the hazard is not severe, the maximum deficiency that can occur is a single deficiency which may be countered by either a fire resistive enclosure or automatic extinguishing equipment.

A single deficiency situation will also be considered to exist when a severe hazard is either protected by automatic extinguishing systems or by fire resistive enclosure but not by both.

The term "Adjust Zone" as used in the evaluation form means any zone, either on the same floor or on the floor immediately below, that physically 
abuts the zone being evaluated and is not separated by one-hour fire resistive rated construction.

Table D-4 is abstracted from paragraph 10-1371 (10-2.3.5.1) of the 1973 (1976) Life Safety Code and provides guidance in identifying hazardous and severely hazardous areas.

\section{Smoke Control}

Smoke Control - The smoke control definitions are as follows:

A. No control. There are no smoke barriers (or horizontal exits) on the floor and there is no mechanical smoke control system.

B. Smoke partition. A smoke partition consists of a partition extending across the entire width of the zone equipped with doors that are either self closing or are closed upon detection by smoke detectors located at the door arches or other release mechanism as described in paragraph 5-2134 (5-2.1.2.3). To be credited as a smoke partition an existing partition must also conform with the requirements of paragraphs 10-2312 through 10-2315 (10-3.3.6.2 - 10-3.3.6.5) of the 1973 (1976) Life Safety Code. New smoke partitions in either new or existing buildings must meet the more stringent requirements of paragraphs 10-1313 through 10-1318 (10-3.3.6.6.3 - 10-3.3.6.6.8) of the Life Safety Code. A horizontal exit will act as a smoke partition and is credited as both a smoke partition (item 9) and an emergency movement route (item 10).

C. Mechanically assisted systems* - by zone. Mechanically assisted smoke control on a zone basis must include a smoke partition as in B above supported by a mechanism of automatic controlled fans, smoke vent shafts, or a combination thereof to provide a pressure differential that will assist in confining the smoke to the zone of origin. The fans involved may be special smoke control fans or special adjustment of the normal building air movement fans.

D. Mechanically assisted systems* - by corridor. Mechanically assisted smoke control on a corridor basis is a system initiated by a method of smoke detection that will assure operation of the smoke control system before significant smoke has entered into the corridor of the zone. The mechanism must be capable of pressurizing the corridor in the zone sufficiently to prevent smoke from the room of origin entering the corridor through the entire course of the fire. For such a system to be effective it must be able to hold back the smoke through the expected maximum severity of the fire. This type of smoke control system must also have the capability of evacuating smoke from the corridor on the presumption that the emergency

*Paragraphs 10-2326 (10-3.3.7.1) and 10-1325 (10-2.3.7.1) require that sleeping rooms have operable outside windows. This requirement does not apply where mechanically assisted systems are provided. 
evacuation procedures and other activities involving the opening and closing of doors will cause occasional brief periods of overpowering of the smoke control system and movement of smoke from the fire area into the corridor. This would normally necessitate early warning smoke detectors, automatic door closers, and/or sprinklered protection. Where such is the case the individual credits for each of the involved protection devices are additive to the credits for the smoke control system.

\section{Emergency Movement Routes}

A movement route is any means of egress meeting the requirements for such means in Sections 5-3 (5-2.11) through 5-10 (5-2.7) of Chapter 5 of the 1973 (1976) Life Safety Code excluding only fire escapes, fire escape ladders Section 5-92 (5-2.10) and slide escapes 5-93 (5-2.11). Horizontal exits shall also meet the requirements stated below. Doors exiting directly to the exterior shall also constitute a movement route for the room containing such a door.

A. $\quad \leq 2$ Routes - The emergency movement means from a zone is classified as less than two routes if there are not two or more movement routes serving it. Movement routes may be outside the physical limits of the zone.

B. Multiple Routes - The emergency movement route is multiple if the zone occupants have the choice of two or more distinctly separated movement routes from the zone.

C. Deficient Capacity - The egress route shall be considered as deficient in capacity if the door to a patient room or passage through a smoke barrier is less than 34 inches in the clear or if the corridor in the zone between patient rooms and smoke barriers and exits is less than 48 inches in clear width. These figures are based on the minimum width for a wheelchair to egress a room and the minimum width for the passage of a wheelchair in one direction and an ambulatory person in the opposite direction. Exit routes shall also be considered deficient if any of the dimensional details are less than that required by the Life Safety Code for the egress route involved. However any route where the doors from rooms or through partitions or walls are less than 32 inches in the clear, where the corridor(s) involved are less than 34 inches wide or where stair or stair access is less than 28 inches in the clear shall not be credited as an egress route. Exit routes shall also be considered deficient in capacity if they are not provided with emergency lighting in accordance with Section 10-2271 (10-3.2.10.1).

D. Horizontal Exit - The presence of a single horizontal exit from the zone being evaluated shall be considered meeting this requirement provided the space on the opposite side of the horizontal exit is capable of handling all of the patients from affected zones. To be credited as a horizontal exit the existing arrangement must also conform with the requirements of $10-2261(10-3.2 .2 .5)$ of the 1973 
(1976) Life Safety Code. New horizontal exits in new or existing buildings must meet the more stringent requirements of paragraph 10-1261 (10-2.2.2.5) of the Life Safety Code.

E. Direct Exits - To be credited with direct exits, each patient use space (except bath rooms, restrooms, and corridors) in the zone shall have a door operable by the room occupant(s) that opens directly to the exterior at grade or onto an exterior balcony with direct access to an exterior exit or a smoke proof tower. To be credited, the direct exit must be ramped or otherwise without steps or changes in elevation that would prevent or obstruct the movement of wheelchairs or wheel littered patients through the direct exits to a place of safety and refuge.

11. Manual Fire Alarm

The manual alarm systems for new construction shall be in accordance with the requirements of paragraphs $10-1361$ and $10-1284$ (10-2.3.3.2 through 10-2.3.3.5) (10-2.3.3.8). Existing construction shall be in accordance with paragraph 10-2351 (10-3.3.3.2 and 10-3.3.3.5). Connection to the fire department shall be considered as being met if the fire alarm system is connected directly to the fire department, through an approved central station, or through other means acceptable to the authority having jurisdiction.

\section{Smoke Detection and Alarm}

A detection system as used here is one based on use of smoke detectors. No recognition is given for thermal detectors. The detection system categories are as follows:

A. None - There are no smoke detectors in the zone.

B. Corridor Only - Smoke detectors located in the corridor shall be considered as meeting the requirement if the detectors are within 15 feet of each end of the corridor and not more than 30 feet apart throughout the corridor. All such detectors shall be electrically interconnected with the fire alarm system. If the facility does not have a fire alarm system, no credit shall be given to the detectors unless they include an alarming system that meets the requirements for alarming that would be involved with a manual fire alarm system. This includes audible alarm devices throughout the building.

C. Rooms Only - Smoke detectors shall be considered as meeting this requirement when there is at least one smoke detector in each room occupied or used by patients. In rooms having a dimension in excess of 30 feet additional detectors shall be provided so that detector spacing does not exceed approximately 30 feet. Detectors are not required in restrooms or closets. Detectors intended for operation of door closing mechanisms that are located on the patients' 
side of the door or in the door opening are considered as meeting this requirement for rooms of 500 square feet or less.

D. Corridor and Habitable Spaces - Detection systems installed throughout the corridors of the zone involved and in the habitable spaces (patient rooms, nurses' stations, and other areas basically used for human occupancy) shall be considered as meeting the requirements for a corridor and habitable spaces detection system. Closets, toilet rooms, and other auxiliary spaces as well as ceiling voids, interstitials and other building space not used by humans as a normal part of their regular occupancy are not required to have detectors.

E. Total Spaces - Total space provision of detectors includes detector coverage of all spaces except noncombustible building voids which contain no combustible materials. The total space credit is to be given if the zone measured meets this criteria regardless of the presence or lack of detectors in other portions of the building.

\section{Automatic Sprinklers}

In evaluating sprinkler protection within the zone, the protection or lack of protection of hazardous areas is considered separately and covered under safety parameter 8. For all other areas in the zone, sprinklers shall be graded on the following basis:

A. None - No charge is applied if there are no sprinklers or if sprinklers, though present, are not sufficient to qualify for one of the other categories listed herein.

B. Corridor* - The safety parameter value of 2 for corridor sprinklers is to be awarded only where the corridor is protected by a specially designed corridor system in which the sprinkler heads are placed according to their individual spray patterns and in consideration of the flow hydraulics of the sprinkler system. This special design must assure that there is an automatic sprinkler head outside each patient room so positioned that the spray pattern from that head will completely cover the entrance to the protected doorway. This degree of sprinkler protection is based on the amount of sprinkler protection in the measured zone whether or not areas outside the fire zone are similarly protected.

C. Corridor and Habitable Space** - This credit is conditional on the classification of construction type as covered in Safety Parameter Item 1, Construction, as follows:

*Corridor sprinkler protection is credited only in buildings classified as "protected" or "fire resistive" in Safety Parameter Item 1, Construction.

**Habitable space includes patient rooms, nurses' stations, and other areas basically used for human occupancy. Habitable space does not include closets, bathrooms and toilets, elevators, and similar spaces. 
1. Item 1, Construction, is based on a "Protected" or "Fire Resistive" type of construction. This credit is based on a system that effectively provides coverage for all corridor and habitable space in the zone plus the establishment of spray patterns that will protect the doors or other openings to areas not so protected in a manner that will prevent advance of any fire into the sprinklered zone. In buildings of protected or fire resistive construction, the credit is to be applied to any zone where the above conditions are met whether or not areas outside the zone are similarly protected.

2. Item 1, Construction, is based on an "Unprotected" type of construction. In any unprotected type of construction the credit for corridor and habitable space protection is to be given only if, in addition to the conditions described in (1) above, sprinkler protection is also provided in all spaces in the building (including attic or loft spaces) with construction elements that are not sheathed, enclosed or otherwise protected with fire resisting materials such as gypsum board, plaster, or masonry block.

D. Total Space - Total space automatic sprinkler protection is to be credited only if the entire structure is protected by automatic sprinklers in accordance with Section 6-41 (6-4.1) of the 1973 (1976) Life Safety Code.

Wherever sprinkler protection is involved in an area having an unprotected vertical opening, the sprinkler protection around that vertical opening must conform with one of the approved methods outlined either in Chapter 6 of the Life Safety Code or in NFPA Standard No. 13. This protection is required to allow the credit for sprinkler protection but shall in no way reduce any change under safety parameter 7 resulting from an unprotected vertical opening.

In Table 5 of the Fire Safety Evaluation Form, (Individual Safety Evaluations) the value for sprinkler protection credited to the people movement safety $\left(S_{3}\right)$ category is divided by 2 . This produces a safety value only $1 / 2$ the value credited in other categories. This is in recognition of the ability of sprinkler protection to control, confine, and frequently extinguish fire while recognizing its limitation in preventing or controlling the spread of smoke.

Each sprinkler system shall be provided with supervision. Each sprinkler system shall be electrically interconnected with the fire alarm system and the main sprinkler control valve shall be electrically supervised so that at least a local alarm shall sound in a constantly attended location when the valve is closed. 
The construction types are defined per NFPA 101 reference to NFPA 2201961 edition. They consist of:

a. Wood Frame Construction (Unprotected)

Definition - That type of construction in which exterior walls, bearing walls and partitions, floor and roof constructions and their supports are of wood or other combustible material, when the construction does not qualify as Heavy Timber Construction, Ordinary Construction, or Protected Wood Frame Construction.

b. Protected Wood Frame Construction

Definition - Wood Frame Construction may be designated Protected Wood Frame Construction when roof and floor construction and their supports have one-hour fire resistance, and stairways and other openings through floors are enclosed with partitions having one-hour fire resistance.

c. Ordinary Construction (Unprotected)

Definition - That type of construction in which exterior bearing walls or bearing portions of exterior walls are of noncombustible construction having a minimum fire resistance of two hours and stability under fire conditions; non-bearing exterior walls are of noncombustible construction; and in which the roofs, floors and interior framing are wholly or partly of wood (or other combustible material) of smaller dimensions than required for Heavy Timber Construction. Fire resistance may be required for non-bearing exterior walls, and fire resistance additional to that specified may be required for bearing walls or bearing portions of walls, by conditions such as occupancy, location with respect to lot lines, fire exposure and other pertinent conditions.

d. Protected Ordinary Construction

Definition - Ordinary Construction may be designated Protected Ordinary Construction when roof and floor construction and their supports have one-hour fire resistance, and stairways and other openings through floors are enclosed with partitions having one-hour fire resistance.

e. Noncombustible Construction (Unprotected)

Definition - That type of construction in which the walls partitions and structural members are of noncombustible construction not qualifying as Fire Resistive Construction or Protected Noncombustible Construction. 


\section{Table D-1 Construction Types (Continued)}

f. Protected Noncombustible Construction

Definition - Noncombustible Construction may be designated Protected Noncombustible Construction when bearing walls or bearing portions of walls, exterior or interior, are of noncombustible construction having a minimum fire resistance rating of two hours and are stable under fire conditions; roof and floor construction and their supports have one-hour fire resistance; and stairways and other openings through floors are enclosed with partitions having one-hour fire resistance.

g. Fire - Resistive Construction

Definition - That type of construction in which the structural members including walls, partitions, columns, floor and roof constructions are of noncombustible materials with fire resistance ratings not less than those specified in the following table.

The two classifications are identified by the required fire resistance of floors as a matter of convenience. 
Fire Resistance Rating of Structura1 Members in Hours

Bearing walls or bearing portions of walls, exterior or interior

Bearing walls and bearing partitions

must have adequate stability under

fire conditions in addition to the

specified fire resistance rating.

Nonbearing walls or portions of walls, exterior or interior

NC-Noncombustible. Fire resistance may be required in such walls by conditions such as fire exposure, location with respect to lot lines, occupancy or other pertinent conditions.

Principal supporting members including columns, trusses, girders and beams for one floor or roof only

Principal supporting members including columns, trusses, girders and beams for more than one floor or roof

Secondary floor construction members, such as beams, purlins and slabs, not affecting the stability of the building

Secondary roof construction members, such as beams, purlins and slabs, not affecting the stability of the building
Classification

3-hour 2-hour

4

3

NC

NC

3

2

4

3

3

2

2

$11 / 2$ 
Table D-2 Flame Spread Classification for Wall and Ceiling Finishes

Flame Spread - The surface flame spread rating of ceilings and walls as measured by ASTM E-84. The categories of flame spread are:

Class A Interior Finish. Flame Spread 0-25. Includes any material classified at 25 or less on the test described above. Any element thereof when so tested shall not continue to propagate fire.

Class B Interior Finish. Flame Spread 26-75. Includes any material classified at more than 25 but not more than 75 on the test described above.

Class C Interior Finish. Flame Spread 76-200. Includes any material classified at more than 75 but not more than 200 on the test scale described above.

Existing floor covering materials in existing buildings are not to be considered in classification of interior finish. Floor covering material in new buildings shall be considered as Class A in rating interior finish in Table 4, Items 2 and 3, if they are of either Class A or Class B rating. If not the rating shall be based on the actual class of the floor covering material.

No change or adjustment in the above classifications is to be made even if the space is sprinkler protected. The impact of sprinkler protection is separately accounted for in Item 13 of figure 4. 
Transfer grills, whether protected by fusible link-operated dampers or not, shall not be used in these walls or doors. Fixed wired glass vision panels may be placed in corridor walls, provided they do not exceed 1,296 square inches in size and are installed in approved steel frames. Fixed wire glass vision panels may be installed in wood doors, provided they do not exceed 720 square inches in size and are installed in approved steel frames.

Waiting areas of 250 square feet or less on an institutional sleeping floor may be open to the corridor, provided that they are located to permit direct supervision by the institutional staff. Such areas shall be equipped with an electrically supervised automatic smoke detection system installed in accordance with paragraph 10-1362 of the Life Safety Code. Not more than one such waiting area is permitted in each smoke compartment.

Waiting areas of 600 square feet or less on floors other than institutional sleeping floors may be open to the corridor, provided that they are located to permit direct supervision by the institutional staff and so arranged as not to obstruct any access to required exits. Such areas shall be protected by an electrically supervised automatic smoke detection system installed in accordance with paragraph 10-1362 (10-2.3.3.6) of the Life Safety Code.

Space for doctors' and nurses' charting, communications, and clerical areas may be open to the corridor.

All new interior walls and partitions in buildings of fire-resistive and noncombustible construction shall be composed of noncombustible materials. 
Hazardous Areas - Hazardous areas include, but are not restricted to, the following. Those areas accompanied by a dagger ( $\uparrow$ ) in the list shall have both separation and a complete extinguishment system. Others shall be protected by either separation or a complete extinguishment system.

\section{Hazardous Areas}

$\begin{array}{ll}\begin{array}{l}\text { Boiler and heater rooms } \\ \text { Laundries }\end{array} & \begin{array}{r}\text { of coms or spaces used for storage } \\ \text { Kitchens }\end{array} \\ \text { Repair shops } & \text { hazardous by the authority having } \\ \text { Handicraft shops } & \text { jurisdiction } \\ \text { Employee locker rooms } & \text { Trash collection rooms } \\ \text { † Soiled linen rooms } & \text { Gift shops } \\ \text { +Paint shops } & \end{array}$

NOTE: The fire hazard potential of pharmacies, laboratories, and other Medicaid support activities can vary widely from nonhazardous to extra hazardous. The individual cases must be evaluated on the basis of the guidance in section 6-5 of the Life Safety Code and any applicable standards for the specific operation or hazard involved. 

APPENDIX E

SAMPLE COMPLETED FORMS

E-1 
FIGURE E - IA

FIRE/SMOKE ZONE* EVAIUATION WORK SLIEET FOR HEALTH CARE FACIIITIES FACILITY LARGE HOSPITAL_ BUII.DING WHITE

ZONE(S) FVALUATED 3RD FLOOR - C WING

EVALUATOR A.J.S. DATE G-15-78

Complete this work sheet for each enne. Where conditions are the same in several zones, one work sheet can be used for those zones.

Step 1: Determinc Occupancy Rish Parancter factors - Ise lable 1.

A. For each Rish i'aranteter in lable I, select and circle the appropriste rish factor baluc. Choose only onc for each of the five Rish Parameters.

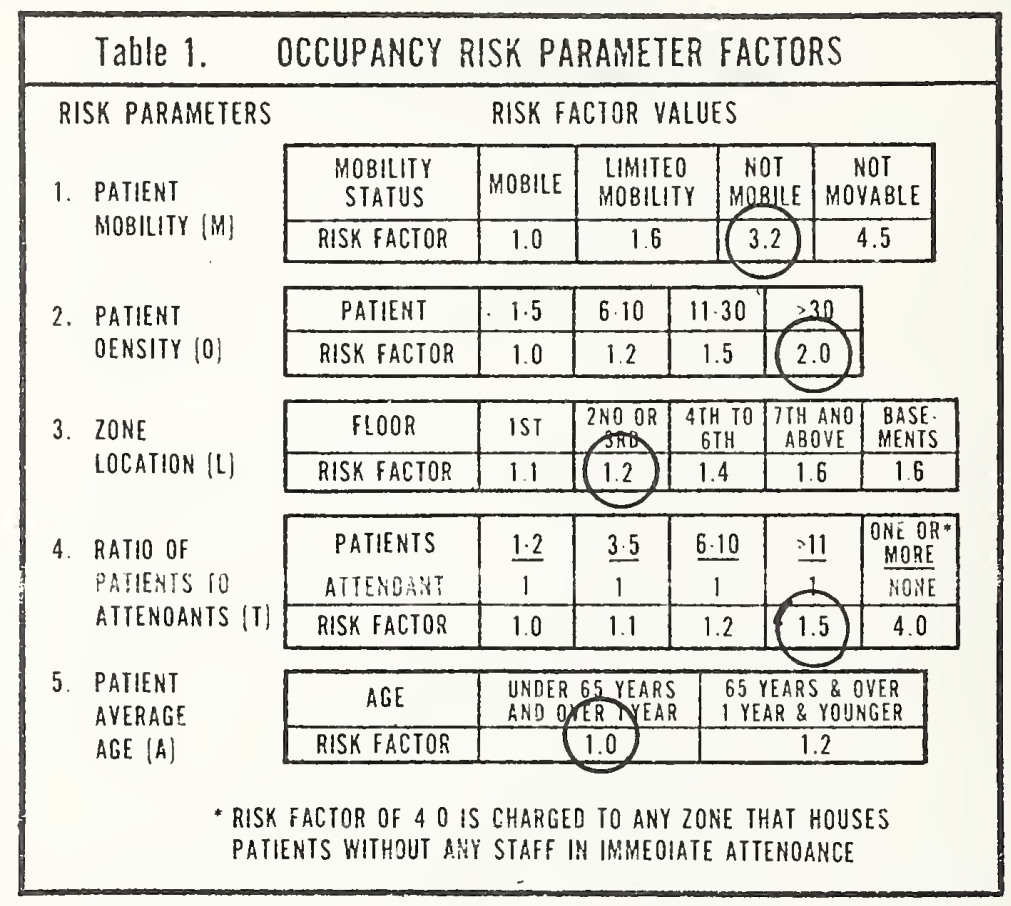

Step 2: Compute Occupancy Risk Factor (F) - Use Table 2.

A. Transfer the circled risk factor values from Table 1 to the corresponding blocks in Table 2.

B. Compute $\mathrm{F}$ by multiplying the risk factor values as indicated in Table 2 .

\section{Table 2. OCCUPANACY RISK FACTOR CALCULATION}

\begin{tabular}{|c|c|c|c|c|c|c|c|c|}
\hline & $M$ & & D & $L$ & & $T$ & A & $\mathrm{F}$ \\
\hline DECUPANCY RISK & 3.2 & $x$ & 2. & $\times 1,2$ & $x$ & $1.5 \times$ & 1. & 11.5 \\
\hline
\end{tabular}

Step 3: Compute Adjusted Building Status (R) - Use Table 3A or 3B.

A. If building is classified as NEW use Table $3 \mathrm{~A}$. If building is classified as existing use Table 3B.

B. Transfer the value of $F$ from Table 2 to Table $3 \mathrm{~A}$ or Table $3 \mathrm{~B}$ as appropriate. Calculate "R."

C. Transfer " $R$ " to the block labeled " $R$ " in Table 7 on page 4 of the work sheet.

Table 3A. (NEW BUILDIVGS)

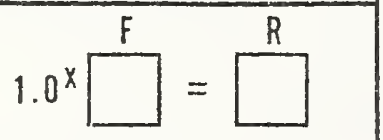

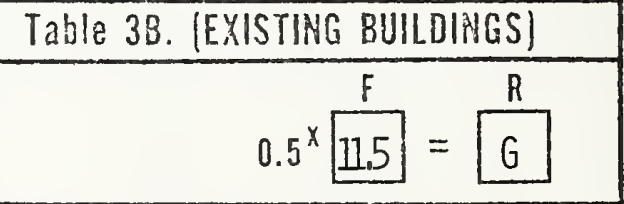

Table 38. (EXISTING BUILDINGS)

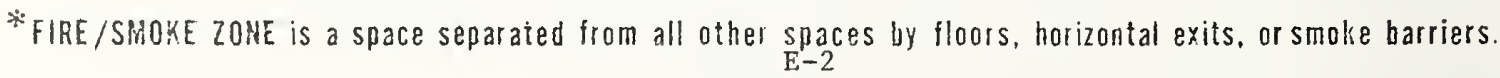


FIGURE E- $1 \mathrm{~B}$

Step 4: Determine Safety Parameter Values - Use Table 4.

A. Select and circle the safety value for each safety parameter in Table 4 that best describes the conditions in the zone. Choose only one value for each of the 13 parameters. If two or more appear to apply choose the one with the lowest print value.

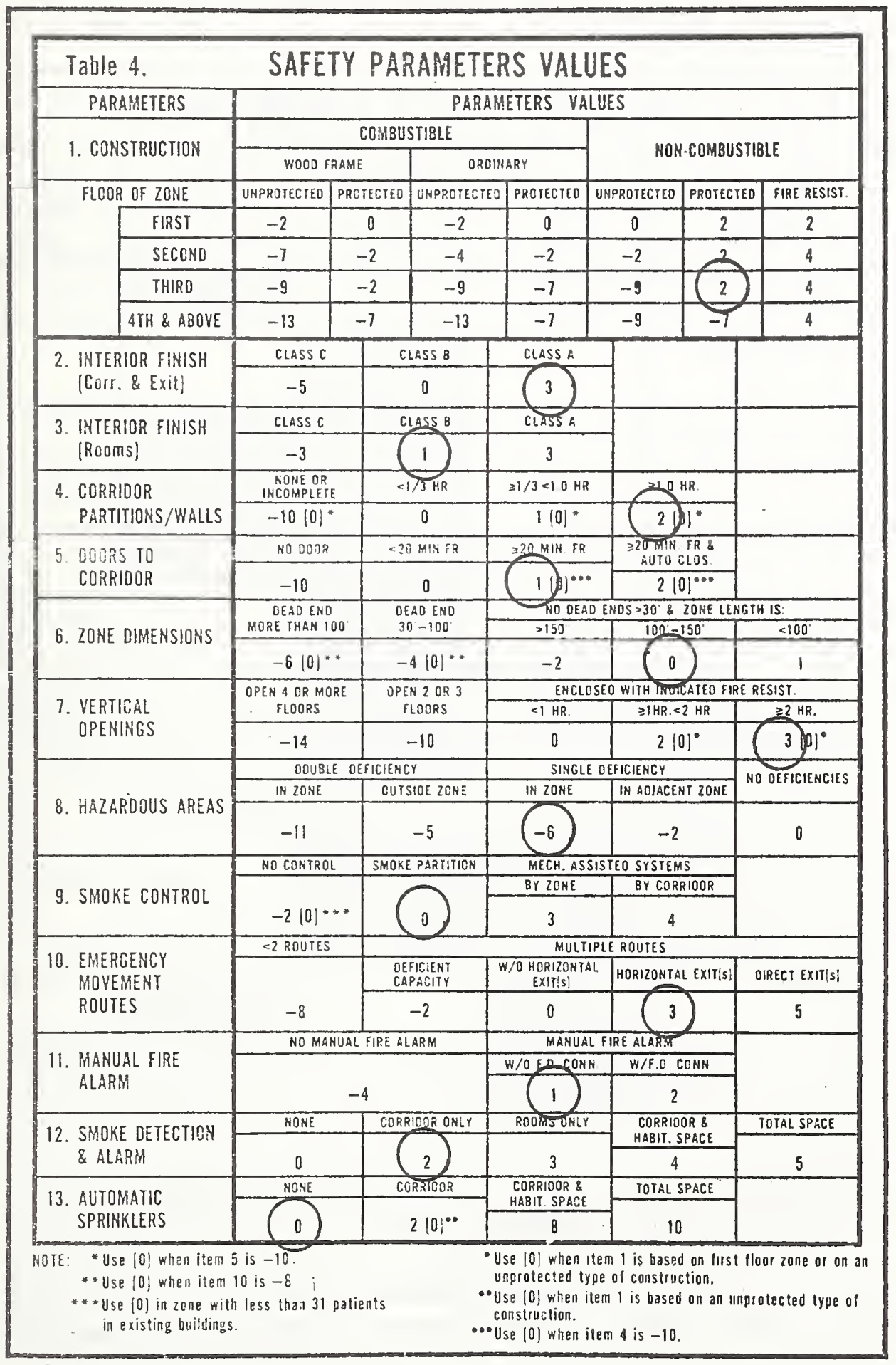


Step S: Compute Individual Safety Evaluations - Use Table 5.

A. Transfer each of the 13 circled Satcty Paraneter Value from Table 4 to every unshaded blocks in the line with the corresponding Safety larameter in Tiable 5. For Safety Parameter 13 (Sprinklers) the value entered in the (People Movement Safety) is recorded in Table 5 as 1.2 the corresponding value circled in Table 4.

B. Add the four columns, keeping in nind that any negative numbers deduct.

C. Transfer the resulting total values for $S_{1}, S_{2}, S_{3}, S_{6}$ to the blocks labeled $S_{1}, S_{2}, S_{3}, S_{1}$ in Table 7 on page 4 of this sheet.

\begin{tabular}{|c|c|c|c|c|}
\hline $\begin{array}{c}\text { SAFETY } \\
\text { PARAMETERS }\end{array}$ & $\begin{array}{c}\text { CONTAINMENT } \\
\text { SAFETY } \\
\text { (Si) }\end{array}$ & $\begin{array}{c}\text { EXTINGUISHMENT } \\
\text { SAFETY } \\
\text { [S2] }\end{array}$ & $\begin{array}{l}\text { PEOPLE } \\
\text { MOVEMENT } \\
\text { SAFETY }\left(S_{3} \text { ) }\right.\end{array}$ & $\begin{array}{l}\text { GENERAL } \\
\text { SAFETY } \\
\text { |SG| }\end{array}$ \\
\hline 1. CONSTRUCTION & 2 & 2 & & 7 \\
\hline $\begin{array}{l}\text { 2. INTERIOR FINISH } \\
\text { (Corr. \& Exit) }\end{array}$ & 3 & & 3 & 3 \\
\hline $\begin{array}{l}\text { 3. INTERIOR FINISH } \\
\text { (ROOMS) }\end{array}$ & 1 & & & 1 \\
\hline $\begin{array}{l}\text { 4. CORRIDOR } \\
\text { PARTITIOAS/WALLS }\end{array}$ & $?$ & & & 2 \\
\hline $\begin{array}{l}\text { 5. DOORS TO } \\
\text { CORRIDOR }\end{array}$ & 1 & & 1 & 1 \\
\hline 6. ZONE DIMENSIONS & & & 0 & 0 \\
\hline 7. VERTICAL OPENINGS & 3 & & 3 & 3 \\
\hline 8. HAZARDOUS AREASS & -6 & -6 & & -6 \\
\hline 9. SMOKE CONTROL & & & 0 & 0 \\
\hline $\begin{array}{l}\text { 10. EMERGENCY } \\
\text { MOYEMENT ROUTES }\end{array}$ & & & 3 & 3 \\
\hline $\begin{array}{l}\text { 11. MANUÁL FIRE } \\
\text { ALARPA }\end{array}$ & & 1 & & 1 \\
\hline $\begin{array}{l}\text { 12. SPAOKE DETECTIDW } \\
\text { \& ALARM }\end{array}$ & & 2 & 2 & 2 \\
\hline $\begin{array}{l}\text { 13. AUTOBATIC } \\
\text { SPRINKLERS }\end{array}$ & 0 & 0 & $\div 2=$ & 0 \\
\hline TOTAL VALUE & $s_{1}=$ & $S_{2}=-1$ & $s_{3}=12$ & $S_{G}=$ \\
\hline
\end{tabular}


Step 6: Determine Mandatory Safety Requirement Values - Use Table 6.

A. Using the classification of the building (i.e., New or Existing) and the floor where the zone is located, circle the appropirate value in cach of the three columns in Table 6.

B. Transfcr the three circled values from Table 6 to the blocks marked $S_{a}, S_{b}$, and $S_{c}$ in Table 7.

\begin{tabular}{|l|c|c|c|c|c|c|}
\hline Table 6. & MANDATORY SAFETY REQUIREMENTS - \\
\hline & $\begin{array}{c}\text { CONTAINMENT } \\
S_{a}\end{array}$ & \multicolumn{2}{|c|}{$\begin{array}{c}\text { EXTINGUISHMENT } \\
S_{b}\end{array}$} & $\begin{array}{c}\text { PEOPLE MOVEMENT } \\
S_{c}\end{array}$ \\
\hline ZONE LOCATION & New & EXist. & New & Exist. & New & Exist. \\
\hline FIRST FLOOR & 9.0 & 4.0 & 6.0 & 3.0 & 6.0 & 1.0 \\
\hline ABOVE FIRST FLOOR & 14.0 & $(8.0)$ & 8.0 & $(5.0)$ & 9.0 & $(3.0)$ \\
\hline
\end{tabular}

Step 7: Evaluation Fire Safety Equivalency - Use Table 7.

A. Perform the indicated subtractions in Table 7. Enter the differences in the appropriate answer blocks.

B. For each row check "Yes" if the value in the answer block is zero or greater. Check "No" if the value in the answer block is a, negative number.

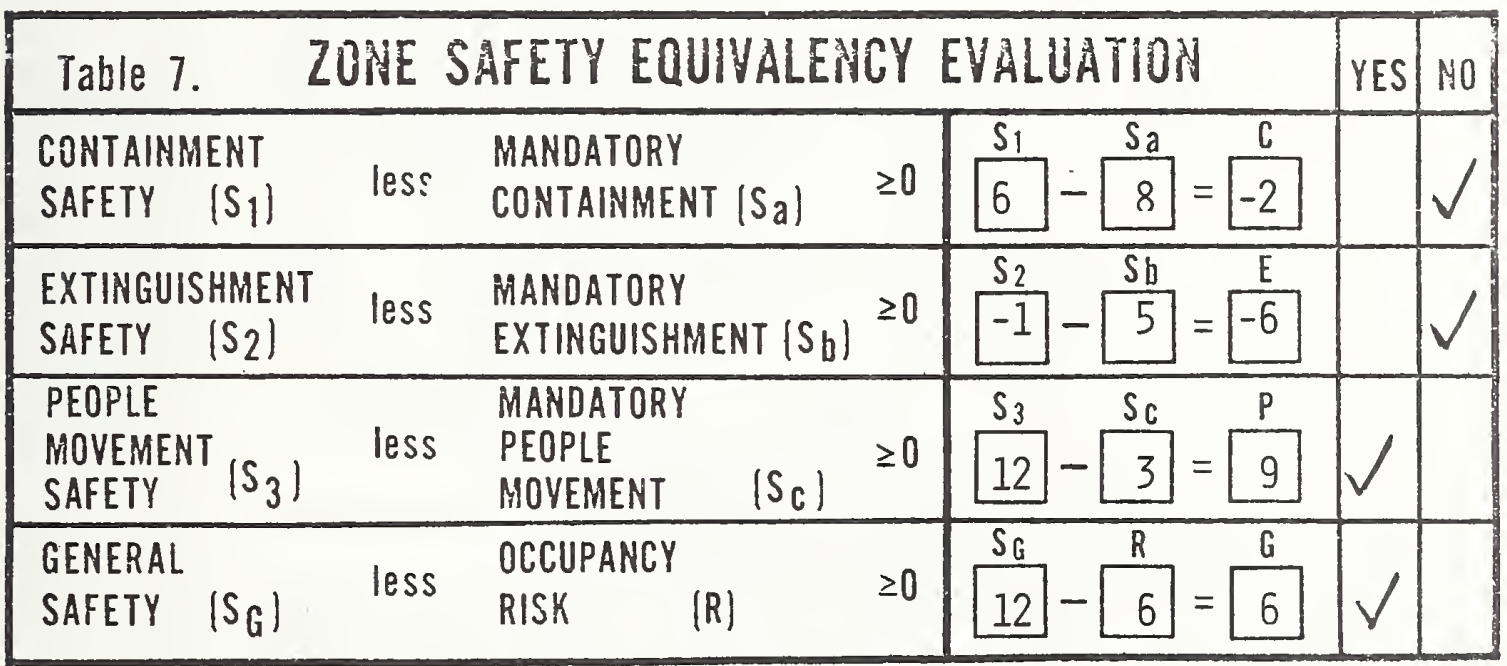

CONCLUSIONS:

1..[ ] All of the checks in Table 7 are in the "Yes" column. The level of fire safety is at least equivalent to that prescribed by the Life Safety. Code*

2. $[X]$ One or more of the checks in Table 7 are in the "No" column. The level of fire safety is not shown by this system to be equivalent to that prescribed by the Life Safety Code."

- The equivalency covered by this worksheet includes the majority of considerations covered by the Lifc Safety Code. There are a few considerations that are not evaluated by this method. These must be separately considered. These additional considerations are covered in the "Facility Fire Safety Requirements Worksheet." One copy of this separate worksheet is to be complcted for each facility.

This form has been prepared by the Fire Safety Engineering Division. Center for Fire Research, NBS, as part of the HEW/NBS Life Safety/Fire Safety projeet. 
FIGURE E - 2A

FIRE/SMOKE ZONE* EVAIUATION WORK SHFET FOR HEALTH CARE FACII.ITIES

FACILITY BUIILDING

ZONE(S) EVALUATED SECOND FLOOR

EVAIUATOR A.」.S. S. DATE 6-15-78

Complete this work sheet for each zone. Where conditions are the same in several zones, one work sheet can be used for those zones.

Step I. Determine Occupancy Rish Parameter Factors - l'se lable I.

A. For each Kisk Parameter in Table I, select and circle the appropriate risk factor valuc. Choose only one for each of the five Risk Parameters.

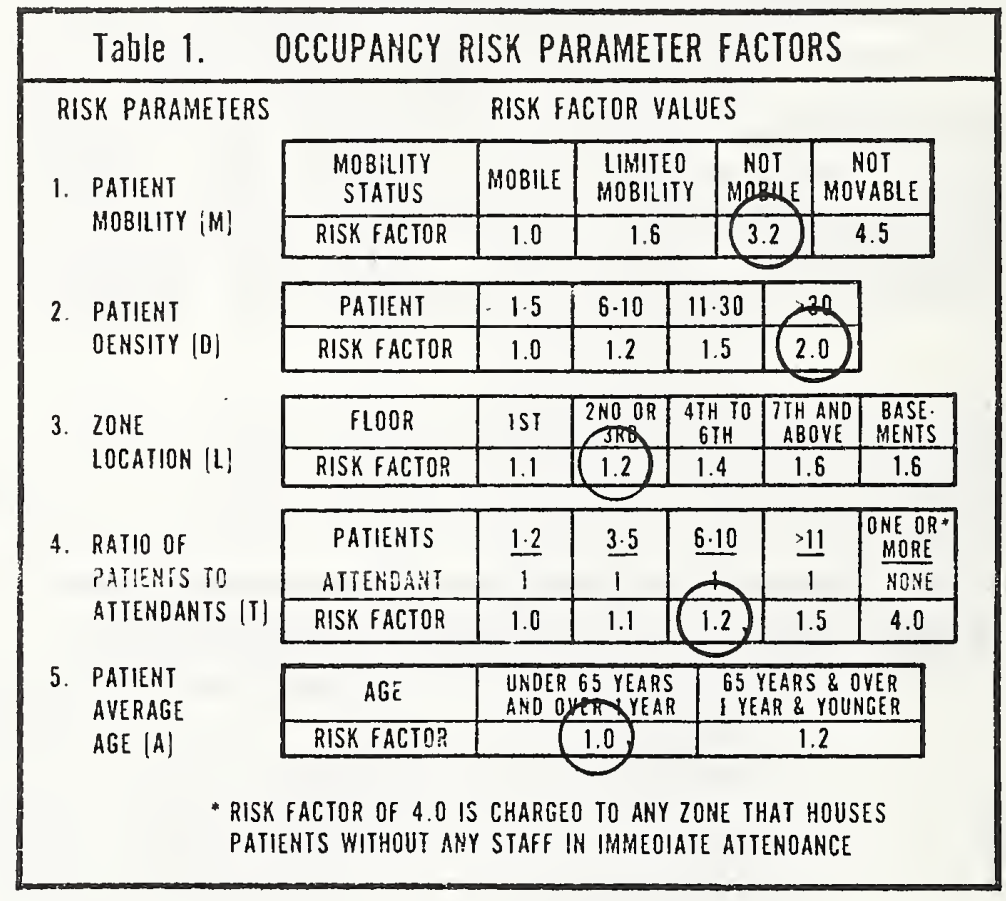

Step 2: Compute Occupancy Risk Factor (F) - Use Table 2.

A. Transfer the circled risk factor values from Table $f$ to the corresponding blocks in Table 2.

B. Compute $F$ by multiplying the risk facto: values as indicated in Table 2.

\section{Table 2. OCCUPANCY RISK FACTOR CALCULATION}

\begin{tabular}{|c|c|c|c|c|c|c|c|c|c|}
\hline & M & & 0 & & $\mathrm{~L}$ & $T$ & & A & $\mathrm{F}$ \\
\hline JCCUPANCY RISK & 3.2 & $x$ & 2 & $x$ & 1.2 & $\times 1.2$ & $x$ & 1 & $0 ?$ \\
\hline
\end{tabular}

Step 3: Compute Adjusted Building Status ( $R$ ) - Use Table 3A or 3B.

A. If building is classified as NEW use Table 3A. If building is classified as existing use Table $3 B$.

$B$. Transfer the value of $F$ from Table 2 to Table $3 A$ or Table $3 B$ as appropriate. Calculate "R."

C. Transfer " $R$ " to the block labeled " $R$ " in Table 7 on page 4 of the work sheet.
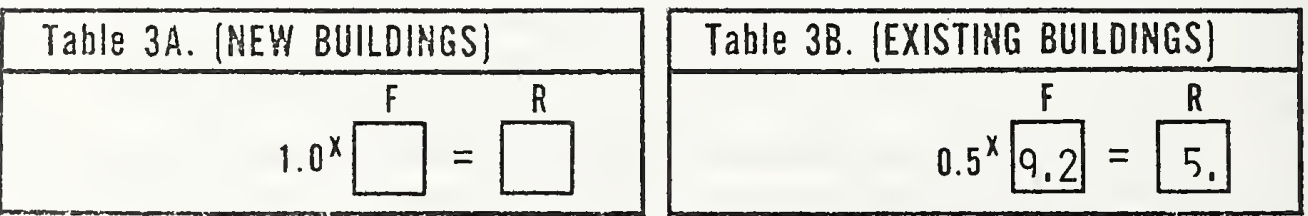

* FIRE/SinCKE ZONE is a space separated from all other spaces by floors, horizontal exits, or smoke barriers. 
Step 4: Determine Safety Parameter Values - Use Table 4.

A. Select and circle the safety value for each safety parameter in Table 4 that best describes the conditions in the zone. Choose only one value for each of the 13 parameters. If two or more appear to apply choose the one with the lowest print value.

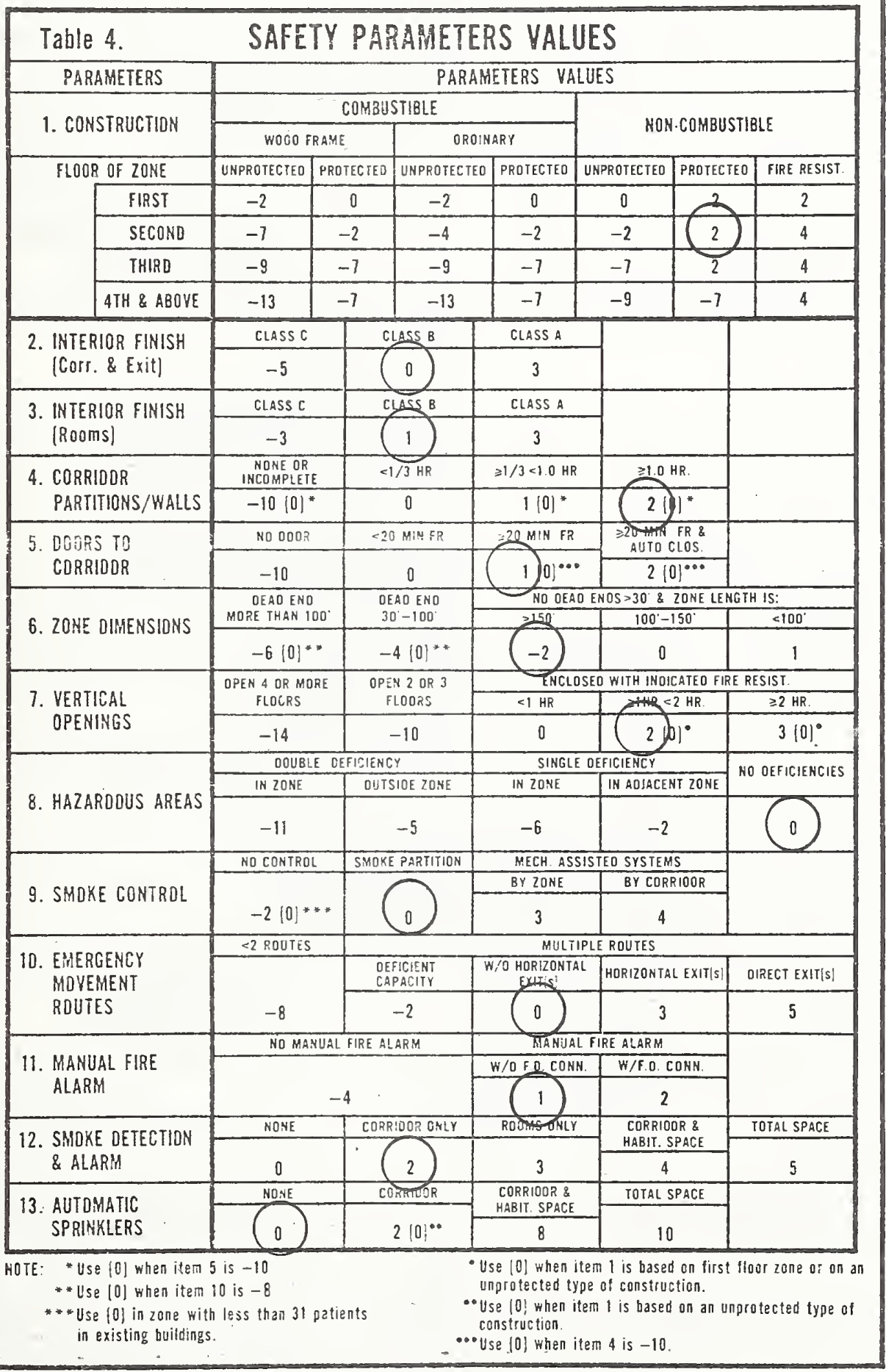


FIGURE $E-2 C$

Step 5: Compute Individual Safety Evaluations - Use Table 5

A. Transfer each of the 13 eireled Safety Parameter Value from Table 4 to cvicry unshaded blocks in the line with the eorresponding Safety Parameter in Iable 5. For Safety Parameter 13 (Sprinklers) the value entered in the (Pcople Movement $S_{a f e t}$ ) is recorded in Table 5 as 1,2 the corresponding value eireled in Table 4.

B. Add the four columns, keeping in mind that any negative numbers deduct.

C. Transfer the resulting total values for $S_{1}, S_{2}, S_{3}, S_{6}$; to the blocks labeled $S_{1}, S_{2} . S_{3} . S_{6}$ in Tatle 7 on page 4 of this sheet.

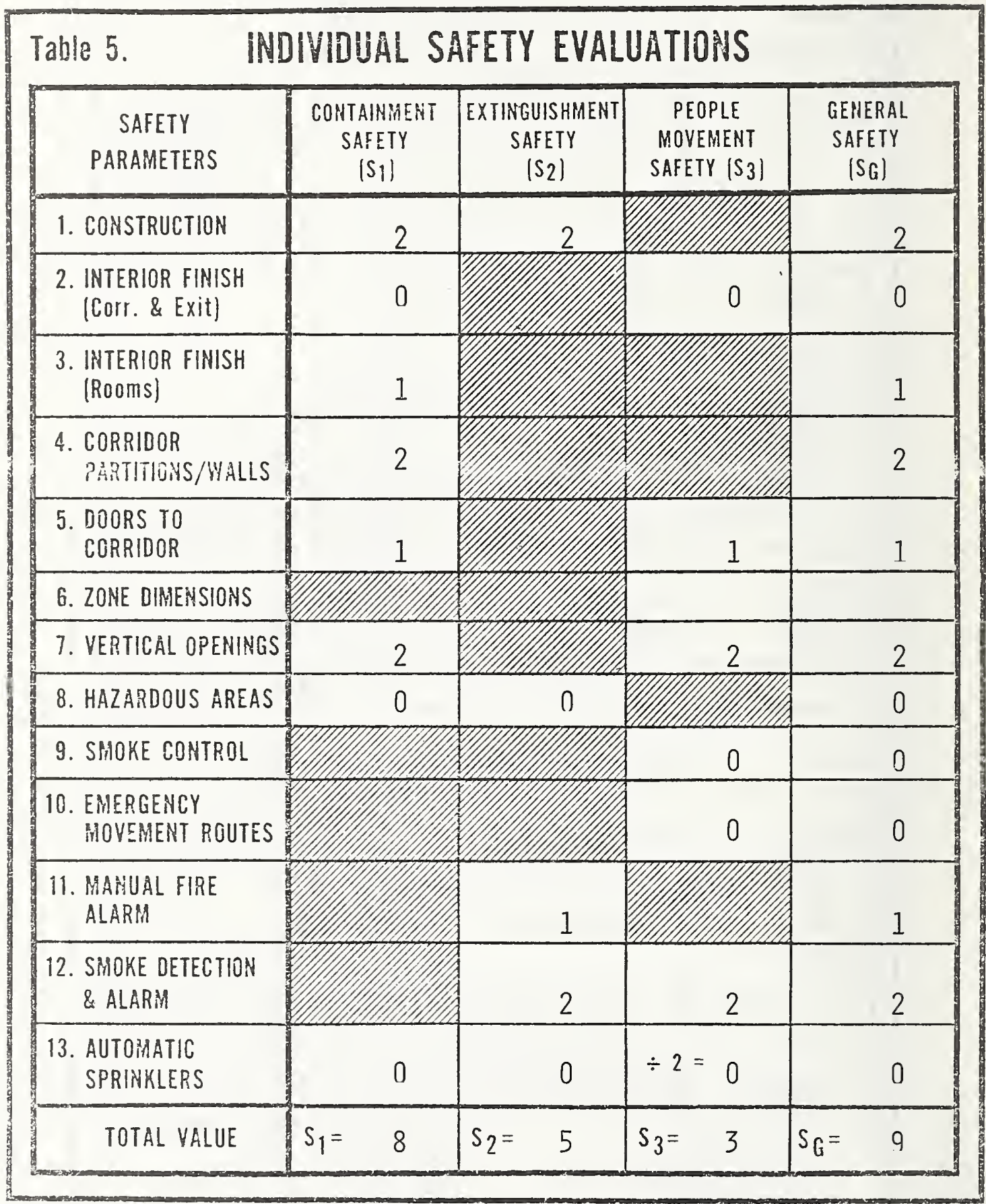


Step 6: Determine Mandatory Safety Requirement Values - Use Table 6.

A. Using the classification of the building (i.e., New or Existing) and the floor where the zone is located, circle the appropirate value in each of the three columns in Table 6.

B. Transfer the three circled values from Table 6 to the blocks marked $S_{a}, S_{h}$, and $S_{c}$ in Table 7.

\begin{tabular}{|c|c|c|c|c|c|c|}
\hline \multicolumn{7}{|c|}{ MANDATORY SAFETY REQUIREMENTS } \\
\hline & \multicolumn{2}{|c|}{$\begin{array}{c}\text { CONTAINMENT } \\
S_{a}\end{array}$} & \multicolumn{2}{|c|}{$\begin{array}{c}\text { EXTINGUISHMENT } \\
\mathrm{S}_{b}\end{array}$} & \multicolumn{2}{|c|}{$\begin{array}{l}\text { PEOPLE MOVEMENT } \\
S_{C}\end{array}$} \\
\hline ZONE LOCATION & New & Exist. & New & Exist. & New & Exist. \\
\hline FIRST FLOOR & 9.0 & 4.0 & 6.0 & 3.0 & 6.0 & 1.0 \\
\hline ABOVE FIRST FLOOR & 14.0 & (8.0) & 8.0 & (5.0) & 9.0 & (3.0) \\
\hline
\end{tabular}

Step 7: Evaluation Fire Safety Equivalency - Use Table 7.

A. Perform the indicated subtractions in Table 7. Enter the differences in the appropriate answer blocks.

B. For each row check "Yes" if the value in the answer block is zero or greater. Check "No" if the value in the answer block is a,negative number.

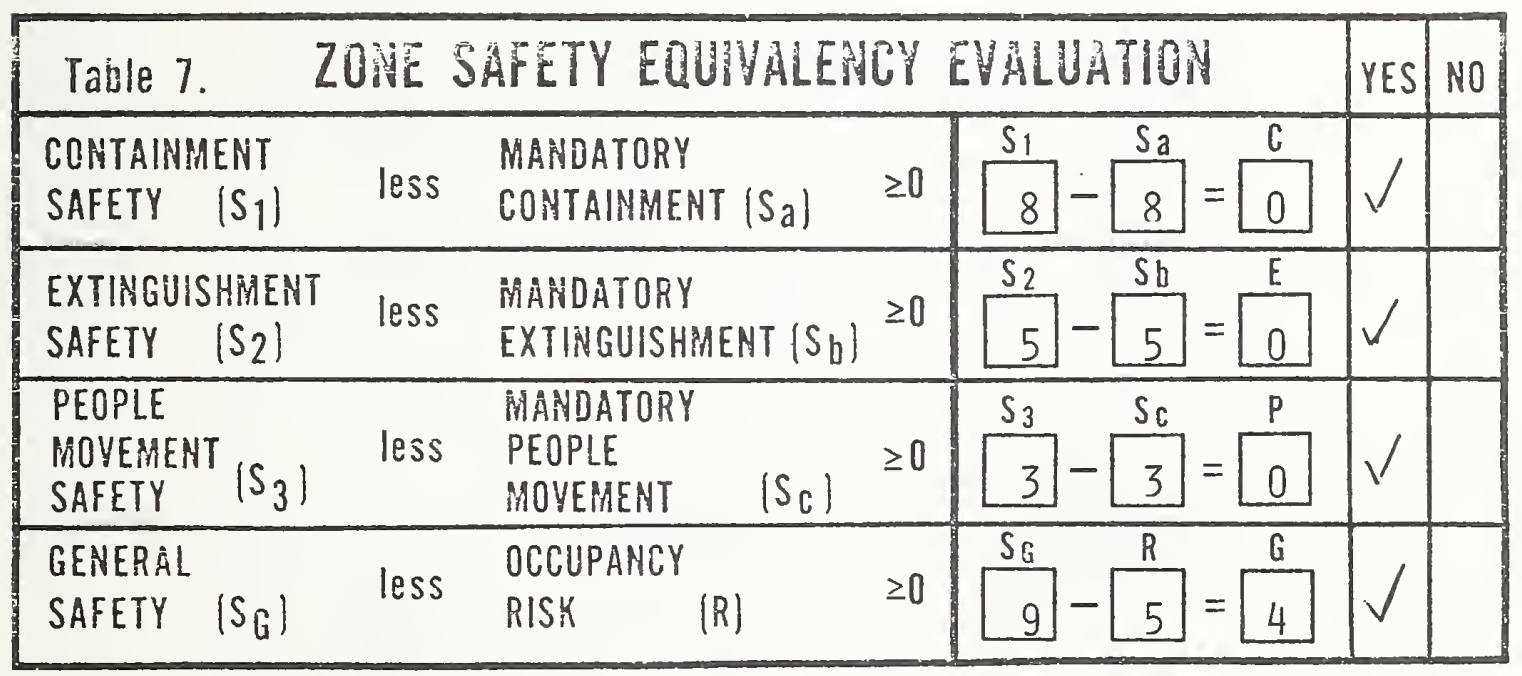

\section{CONCLUSIONS:}

[. [ ] All of the checks in Table 7 are in the "Yes" column. The level of fire safety is at least equivalent to that prescribed by the Life Safety Code*

2. [X] One or more of the checks in Table 7 are in the "No" column. The level of fire safety is not shown by this system to be equivalent to that prescribed by" the Life Safety Code.*

*The equivalency covered by this w'orksheet includes the majority of considerations covered by the Life Safety Code. There are a few considerations that are not evaluated by this method. These must be separately considered. These additional considerations are covered in the "Facility Fire Safety Requirements Worksheet." One copy of this separate workshcet is to be completed for each facility.

This form has been prepared by the Fire Safety Engineering Division. Center for Fire Rcsearch, NBS, as part of the HEW/NBS life Safety/Fire Safety project. 
FIGURE E-3A

FIRE/SMOKE ZONE* EVALUATION WORK SHEET FOR HEALTH CARE FACILITIES

FACILITY NURSING HOME

ZONE(S) EVALUATED FIRST FLOOR

EVALUATOR A.J.S. DATE $6-15-78$

Complete this work sheet for each zone. Where conditions are the same in several zones, one work sheet can be used for those zones.

Step 1: Determine Occupancy Risk Parameter F'actors - I'se Table 1.

A. For each Rish Parameter in lable 1, select and circle the appropriate risk factor value. Choose only one for each of the five Risk Parameters.

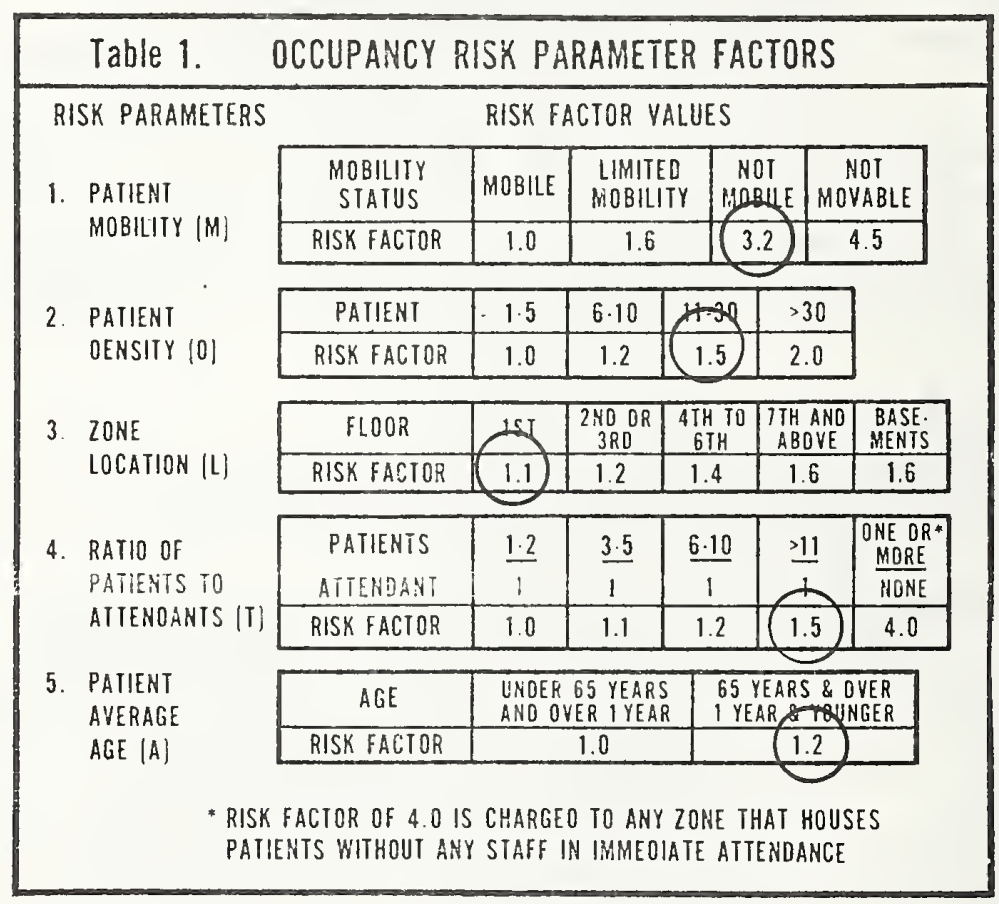

Step 2: Compute Occupancy Risk Factor (F) - Use Table 2.

A. Transfer the circled risk factor values from Table 1 to the corresponding blocks in Table 2 .

B. Compute $\mathrm{F}$ by multiplying the risk factor values as indicated in Table 2 .

Table 2. OCCUPANCY RISK FACTOR CALCULATION

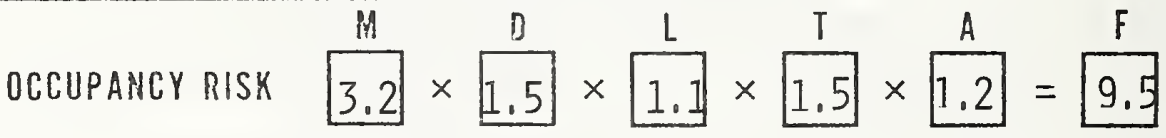

Step 3: Compute Adjusted Building Status (R) - Use Table 3A or 3B.

A. If building is classified as NEW use Tabie 3A. If building is classified as existing use Table $3 \mathrm{~B}$.

B. Transfer the value of $F$ from Table 2 to Table 3.4 or Table $3 B$ as appropriate. Calculate "R."

C. Transfer " $R$ " to the block labeled " $R$ " in Table 7 on page 4 of the work sheet.

Table 3A. (MEW BUILDINGS)

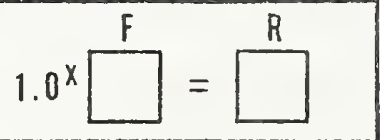

Table 3B. (EXISTING BUILDINGS)

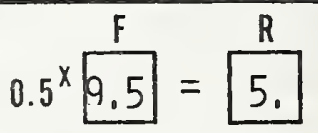

*FIRE/SHIOKE ZONE is a space sepafated from all othef spaces by floors, horizontal exits, or smoke barriers. E-10 
FIGURE E-3B

Step 4: Determine Safety Parameter Values - Use Table 4.

A. Select and circle the safety value for each safety parameter in Table 4 that best describes the conditions in the zone, Choose only one value for each of the 13 parameters. If two or more appear to apply choose the one with the lowest print value.

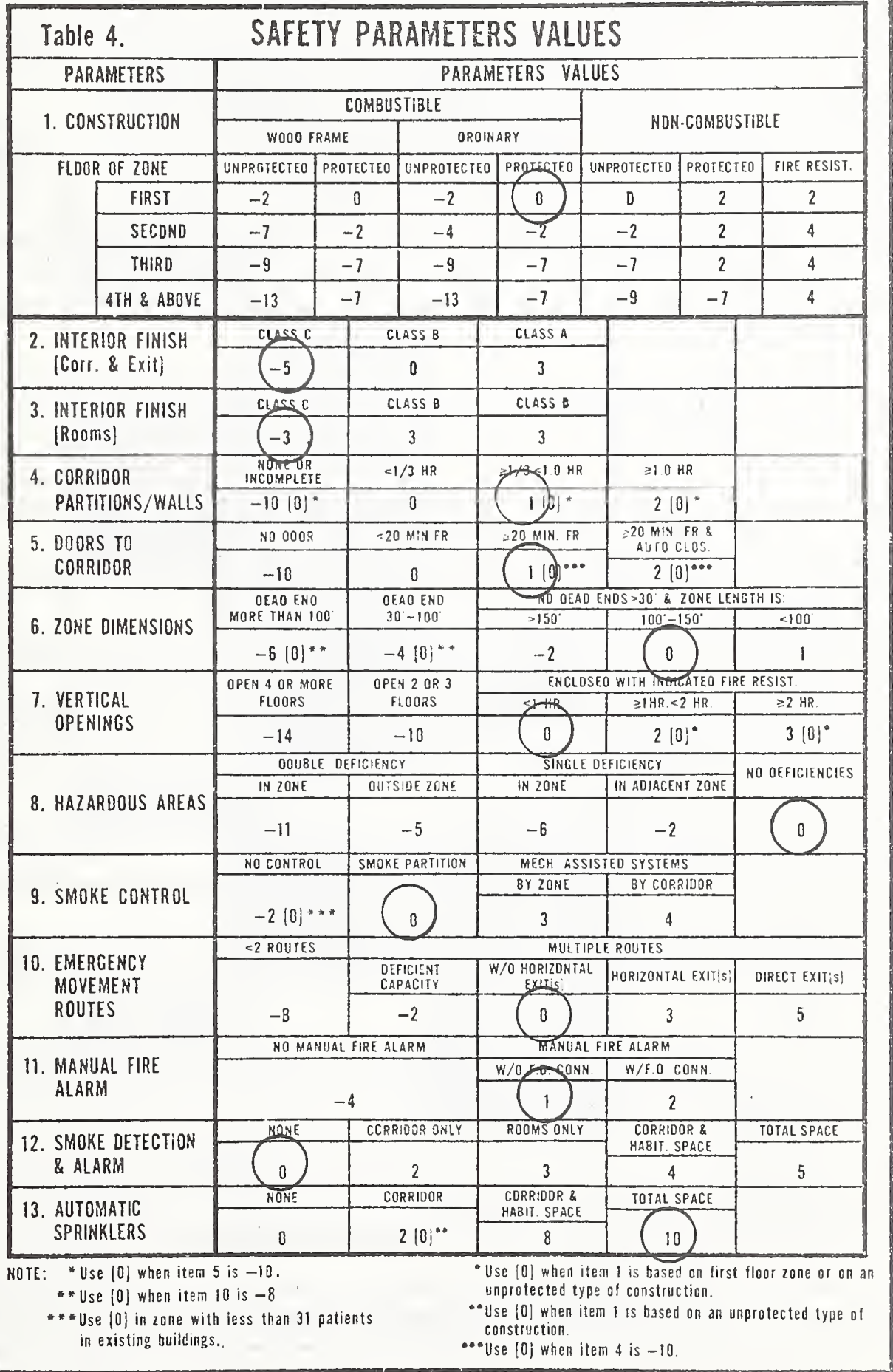


FIGURE E-3C

Step 5: Compute Individual Safety Evaluations - Use Table 5.

A. Transfer each of the 13 circled Satety Parameter Value from Table 4 to every unshaded blocks in the line with the corresponding Safety Parameter in Table 5. For Safety Parameter 13 (Sprinklers) the value entered in the (People Movement Safcty) is recorded in Table 5 as 1/2 the corresponding value circled in Table 4.

B. Add the four columns, keeping in mind that any negative numbers deduct.

C. Transfer the resulting total values for $S_{1}, S_{2}, S_{3}, S_{6}$ to the blocks labeled $S_{1}, S_{2} . S_{3} . S_{6} ;$ in Table 7 on page 4 of this sheet.

\begin{tabular}{|c|c|c|c|c|}
\hline $\begin{array}{c}\text { SAFETY } \\
\text { PARAMETERS }\end{array}$ & $\begin{array}{c}\text { CONTAINMENT } \\
\text { SAFETY } \\
\left(S_{1} \mid\right.\end{array}$ & $\begin{array}{c}\text { EXTINGUISHMENT } \\
\text { SAFETY } \\
\left\{S_{2} \mid\right.\end{array}$ & $\begin{array}{l}\text { PEOPLE } \\
\text { MOVEMENT } \\
\text { SAFETY }\left\{S_{3}\right)\end{array}$ & $\begin{array}{l}\text { GENERAL } \\
\text { SAFETY } \\
\text { [SG] }\end{array}$ \\
\hline 1. CONSTRUCTION & 0 & 0 & & 0 \\
\hline $\begin{array}{l}\text { 2. INTERIOR FINISH } \\
\text { (Corr. \& Exit) }\end{array}$ & -5 & & -5 & -5 \\
\hline $\begin{array}{l}\text { 3. INTERIOR FINISH } \\
\text { (Rooms) }\end{array}$ & -3 & & & -3 \\
\hline $\begin{array}{l}\text { 4. CORRIDOR } \\
\text { PABTITIOAS/WALLS }\end{array}$ & 1 & & & 1 \\
\hline $\begin{array}{l}\text { 5. DOORS TO } \\
\text { CORRIDOR }\end{array}$ & 1 & & 1 & 1 \\
\hline 6. ZONE DIMENSIONS & & & 0 & 0 \\
\hline 7. VERTICAL OPENINGS & 0 & & 0 & 0 \\
\hline 8. HAZARDOUS AREAS & 0 & 0 & & 0 \\
\hline 9. SMOKE CONTROL & & & 0 & 0 \\
\hline $\begin{array}{l}\text { 10. FMERGENCY } \\
\text { MOVEBAENT ROUTES }\end{array}$ & & & 0 & 0 \\
\hline $\begin{array}{l}\text { 11. MABAUAL FIRE } \\
\text { ALAR:A }\end{array}$ & & 1 & & 1 \\
\hline $\begin{array}{l}\text { 12. SHOKE DETECTION } \\
\text { \& ALARM }\end{array}$ & & 0 & 0 & 0 \\
\hline $\begin{array}{l}\text { 13. AUTOMATIC } \\
\text { SPRIAKLERS }\end{array}$ & 10 & 10 & $\div 2=5$ & 10 \\
\hline TOTAL VÁLUE & $s_{1}=$ & $s_{2}=$ & $S_{3}=$ & $S_{G}=$ \\
\hline
\end{tabular}


Step 6: Determine Mandatory Safety Requirement Values - Use Table 6.

A. Using the classification of the building (i.e., New or Existing) and the floor where the zone is located, circle the appropirate value in each of the three columns in Table 6.

B. Transfer the three circled values from Table 6 to the blocks marked $S_{a}, S_{b}$, and $S_{c}$ in Table 7.

\begin{tabular}{|l|c|c|c|c|c|c|}
\hline Table 6. & \multicolumn{6}{|c|}{ MANDATORY SAFETY REQUIREMENTS } \\
\hline & \multicolumn{2}{|c|}{$\begin{array}{c}\text { CONTAINMENT } \\
S_{\mathrm{a}}\end{array}$} & \multicolumn{2}{|c|}{$\begin{array}{c}\text { EXTINGUISHMENT } \\
\text { Sb }\end{array}$} & \multicolumn{2}{|c|}{$\begin{array}{c}\text { PEOPLE MOVEMENT } \\
\text { Sc }\end{array}$} \\
\hline ZONE LOCATION & New & Exist. & New & Exist. & New & Exist. \\
\hline FIRST FLOOR & 9.0 & 4.0 & 6.0 & 3.0 & 6.0 & $(1.0)$ \\
\hline ABOVE FIRST FLOOR & 14.0 & 8.0 & 8.0 & 5.0 & 9.0 & 3.0 \\
\hline
\end{tabular}

Step 7: Evaluation Fire Safety Equivalency - Use Table 7.

A. Perform the indicated subtractions in Table 7. Enter the differences in the appropriate answer blocks.

B. For each row check "Yes" if the value in the answer block is zero or greater. Check "No" if the value in the answer block is a, negative number.

\begin{tabular}{|c|c|c|c|c|c|c|c|}
\hline Table 7. & $E$ & ETY EQUUVAL & & ImLOA & & YES & HO \\
\hline $\begin{array}{l}\text { COHTAINMENT } \\
\text { SAFETY } \quad\left(S_{1}\right)\end{array}$ & less & $\begin{array}{l}\text { MANDATORY } \\
\text { CONTAINMENT }\left(S_{a}\right)\end{array}$ & $\geq 0$ & $\frac{S_{1}}{4}-4$ & $=\frac{C}{0}$ & $\sqrt{ }$ & \\
\hline $\begin{array}{l}\text { EXTINGUUISHMENT } \\
\text { SAFETY }\left(\mathrm{S}_{2}\right)\end{array}$ & less & $\begin{array}{l}\text { HAANDATORY } \\
\text { EXTINGUISHMENT }\left(S_{b}\right)\end{array}$ & $\geq 0$ & $\frac{S_{2}}{11}-\frac{S_{b}}{3}$ & $7=\frac{E}{8}$ & $V$ & \\
\hline $\begin{array}{l}\text { PEOPLE } \\
\text { MOVEMENT } \\
\text { SAFETY }\left(S_{3}\right)\end{array}$ & less & $\begin{array}{l}\text { MANDATORY } \\
\text { PEOPLE } \\
\text { MOVEMENT } \quad\left(S_{C}\right)\end{array}$ & $\geq 0$ & $\begin{array}{l}S_{3} \\
1\end{array}-\frac{S_{c}}{3}$ & $\begin{array}{l}P \\
8\end{array}$ & $\sqrt{ }$ & \\
\hline $\begin{array}{l}\text { GENERAL } \\
\text { SAFETY }\left(S_{G}\right)\end{array}$ & less & \begin{tabular}{ll}
\multicolumn{2}{l}{ OCCUPANCY } \\
RISK & (R)
\end{tabular} & & $\frac{S_{G}}{6}-\frac{R}{5}$ & $=\frac{G}{1}$ & $\sqrt{ }$ & \\
\hline
\end{tabular}

\section{CONCLUSIONS:}

1. [ ] Ai! of the checks in Table 7 are in the "Yes" column. The level of fire safety is at least equivalent to that prescribed by the Life Safety Code*

2. [X] One or more of the checks in Table 7 are in the "No" column. The level of fire safety is not shown by this system to be equivalent to that prescribed by the Life Safety Code."

*The equivalency covered by this worksheet includes the majority of considerations covered by the Life Safety Code. There are a few considerations that are not evaluated by this method. These must be separately considered. These additional considerations are covered in the "Facility Fire Safety Requirements Worksheet." One copy of this separate worksheet is to be completed for each facility.

This form has been prepared by the Fire Safety Engineering Division, Center for Fire Research, NBS, as part of the HEW/NBS Life Safety, Fire Safety' project. 


\section{APPENDIX F \\ COMPUTER PROGRAM}

\section{F-1 Program Description}

The computer program* was developed to support the proof testing efforts of the evaluation system. The program is basically a research tool and not a production tool. It is designed to be used in determining how best to upgrade a health care facility to an acceptable level of safety. The user inputs one or more values for each of the 13 safety parameters. The program examines all possible combinations of these numbers, one value per parameter, and finds those combinations which would upgrade the safety of the facility if implemented. The solutions, or successful combinations, may be sorted by a particular parameter if the user wishes, in which case up to 1000 solutions may be printed for each value of the sorting parameter.

As presently written, the program searches for up to 50000 solutions for each specified value of the sorting routine, limiting the output for each value to at most the first 1000 solutions. A special input card overrides the sorting feature, allowing a change in the total printed output to any number less than 1000 solutions. A limit on the available combinations, or zone configurations, may be accomplished by limiting the number of potential combinations when inputing the parameters, or by choosing "close" upper and lower limits for the total safety values. In the latter method a difference of two points gives a reasonable number of possible solutions. In addition, the special sorting routine provides the capability of anal:zing zone configurations which are limited to certain characteristics.

\section{F-2 Input Requirements}

To operate this program a series of input information is required. The information must be key punched on computer cards. The total number of input cards is 18. The following is a description of the input cards:

Card Number

1

2
Description Title

(Any description as long as it is less than 72 spaces)

Maximum zone configuration printed (any number between 1 and 1000)
Records

In Column 1-72

Begin in Column 1 use digits, do not leave space between the digits. Limited to the number 1000 .

\footnotetext{
*The computer program listing and card deck as well as user instructional information are available upon request from the Center for Fire Research, National Bureau of Standards, Washington, D.C. 20234.
} 
Special Sorting Routine, also called Extended Analysis. (In the same computer run, sorts the zone configuration as a function of level of safety for the parameter which is specified on the input card).

Construction

(see table 4, parameter 1)
Interior Finish

(Corridors \& Exits)

(see table 4, parameter 2)

Interior Finish (Rooms)

Corridor Partition (see table 4, parameter 4)

Doors to Corridor (see table 4, parameter 5)

Zone Lengths (see table 4, parameter 6)

Vertical Openings (see table 4, parameter 7)

Hazardous Areas (see table 4, parameter 8)

Smoke Control (see table 4, parameter 9)

Emergency Movement (see table 4, parameter 10) (see table 4, parameter 3)
No Special Sorting

Zero in column 1 .

Sorting Required

In column 1 the number

of the safety parameter, from table 4, Safety Parameters, Values, of the Fire Zone Safety Evaluation Worksheet.

Beginning in any column, the value of each level to be evaluated, separated by 1 or more blanks. Values must be numeric, may be in any order, and may use (-) for negative values. Do not use (t) for positive values or use decimal points.

Follow the same instruction as in card \#4.

Follow the same instructions as in card 非 4 .

Follow the same instructions as in card $\$ 4$.

Follow the same instructions as in card 非 4 .

Follow the same instructions as in card 非 4 .

Follow the same instructions as in card 非 4 .

Follow the same instructions as in card 非 4

Follow the same instructions as in card 非 4 .

Follow the same instructions as in card 非 4 . 
Manual Fire Alarm (see table 4, parameter 11)

15

16

17

Smoke Detection \& Alarm (see table 4, parameter 12)

Automatic Sprinklers (see table 4, parameter 13)

Minimum Mandatory Values (use values for table 7 - Fire Zone Safety Equivalency Evaluation)

18

Maximum Mandatory Values

The maximum mandatory values must be equal or larger than the minimum mandatory values
Follow the same instructions as in card 非 4 .

Follow the same instructions as in card 非 4 .

Follow the same instructions as in card 非 4 .

Put the value from box $R$, starting in any column, then have 1 or more spaces. Next put in the value from box $5 \mathrm{a}$, and then 1 or more spaces. Next put in the value from box $5 b$, followed by 1 or more spaces. Finally, put in the value from box $5 c$.

Follow the format as defined for card 非17.

\section{F-3 Example}

A typical example of the program output is shown in table F-1. For more information see figures E-IA - E-ID. An analysis was performed to evaluate zone configurations for a new facility. It was assumed that the zone had a dead end which was longer than 100 feet; all hazardous areas were secure and had sprinklers in the corridors and habitable spaces. The zone was on the $4 \mathrm{th}$ floor of the building. The general safety level was estimated to be 15. The minimum containment, extinguishment and people movement safety values were taken from table 6, for a zone located above the first floor in a new building. The maximum safety values were estimated to have a differential value of two points (this provides enough differential for analysis). All zone configurations shown in table F-1 have safety levels which are greater than or equal to the four corresponding specified minimum safety values but which are not all greater than the four corresponding specified maximum safety values. The extended analysis was performed primarily to examine the range of permissible solutions corresponding to five levels of the smoke detection and alarm parameter. 


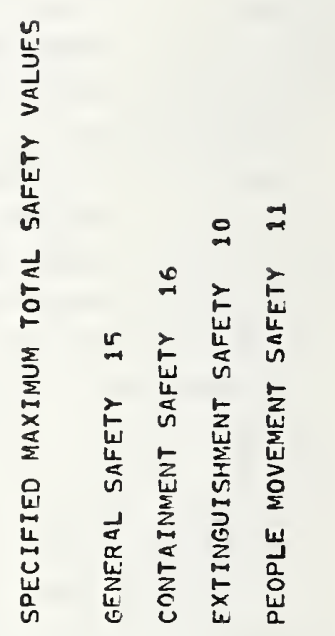

$n$

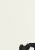

N

㞺

宸 i 


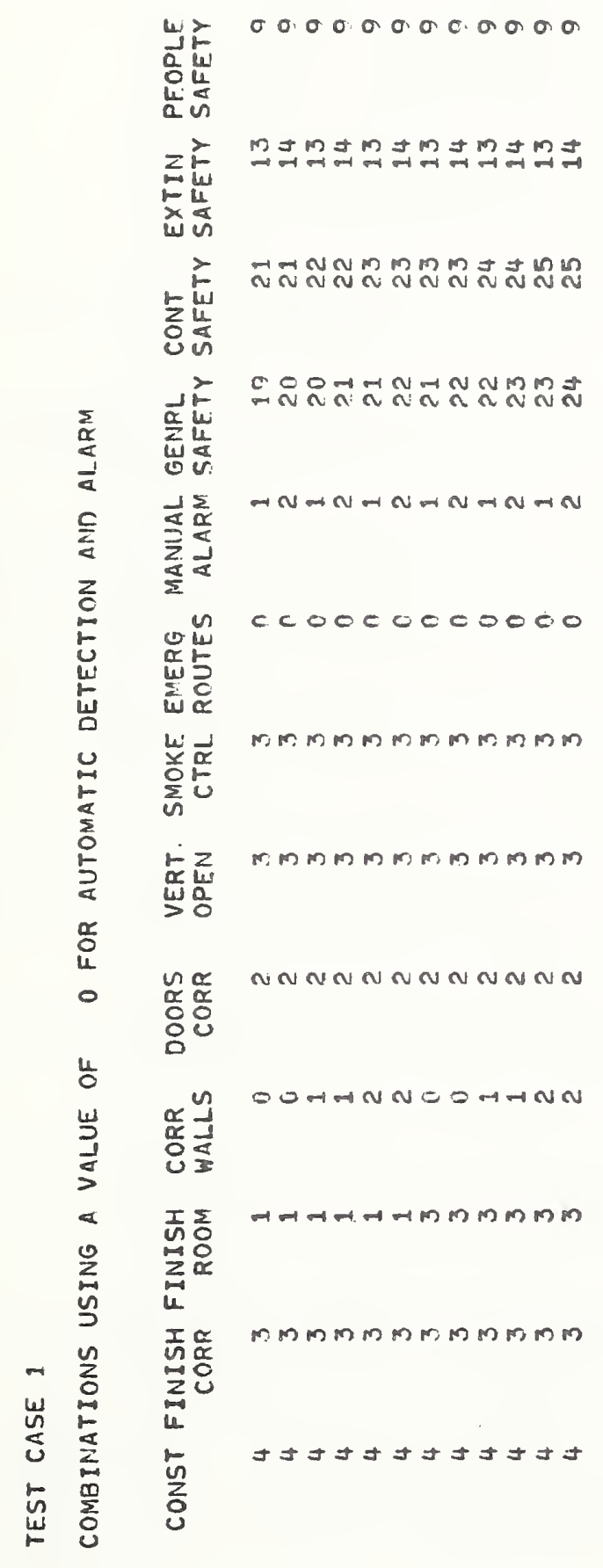




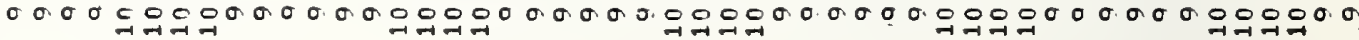

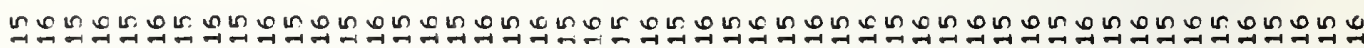

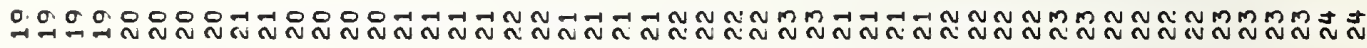

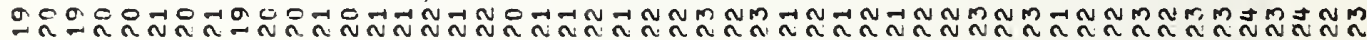
-NAn

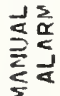
电崖

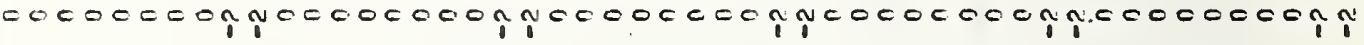
$\sum_{\omega}^{u} \underset{0}{\square}$

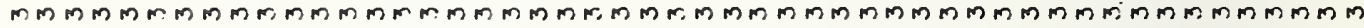
$\sum_{\text {in }}^{\circ}$

$M M N N M M N N M M M M N N M M N N M M F M N N M M N N M M M M N N M M N N M M M M N N M M N N M M$

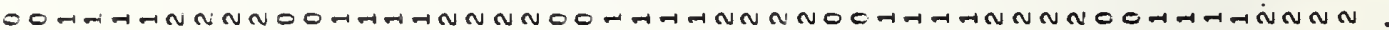
0

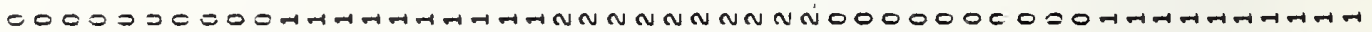
品

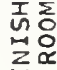

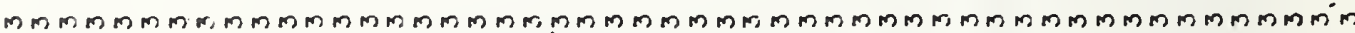
光

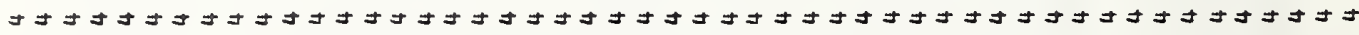
.... 


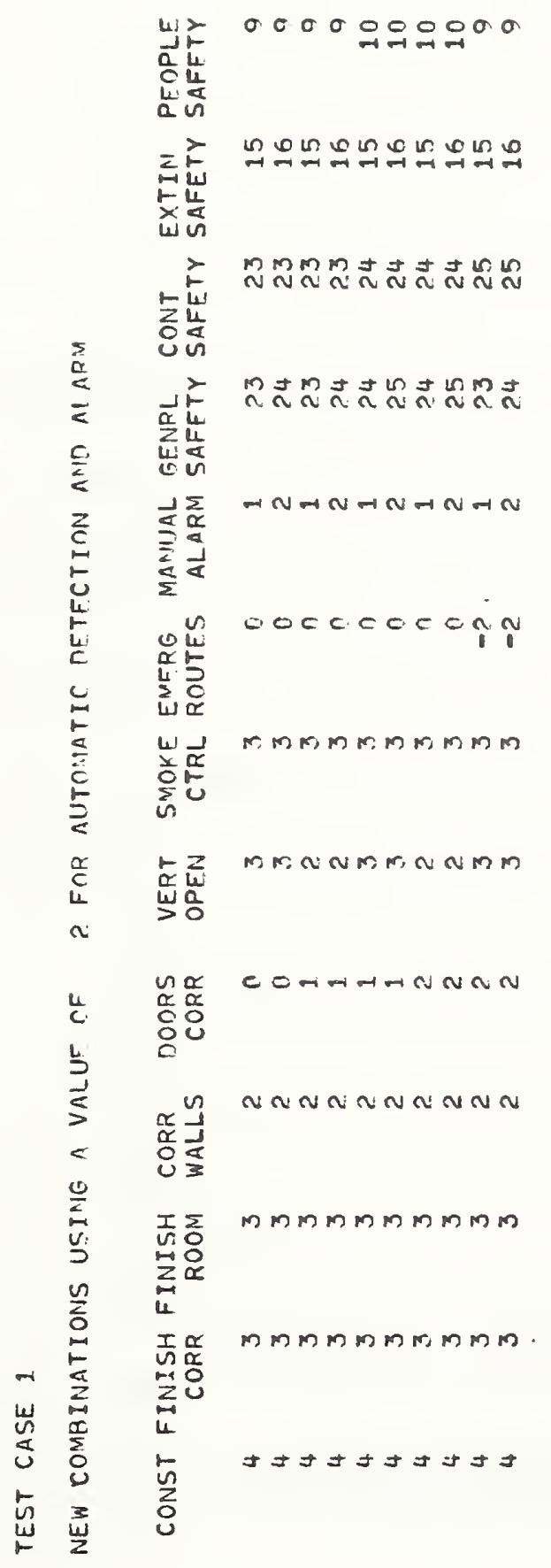




\section{点}

눈

2

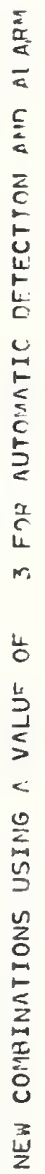

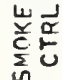

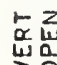
$\tilde{\pi} \frac{x}{\pi}$ 닝 哭 도응 u

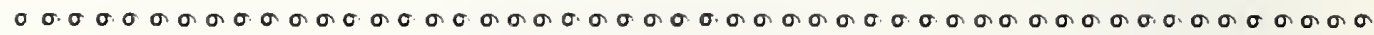

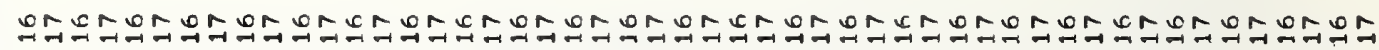

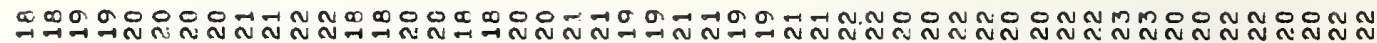

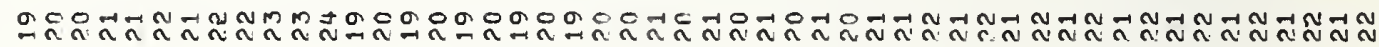
ANAN

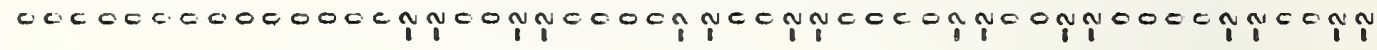
MMMMMMMMRM

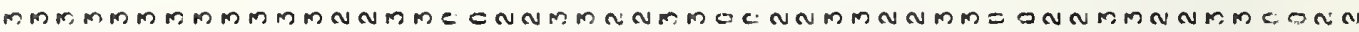

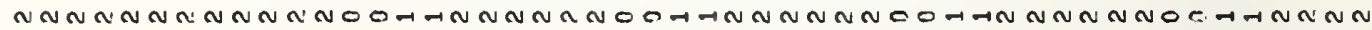

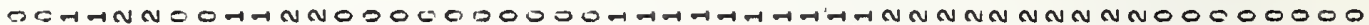

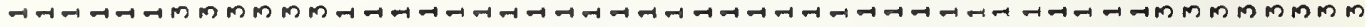

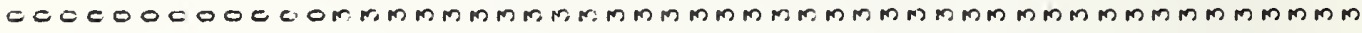

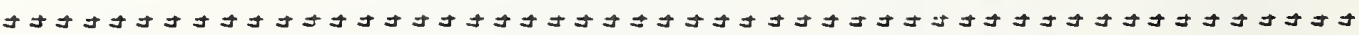




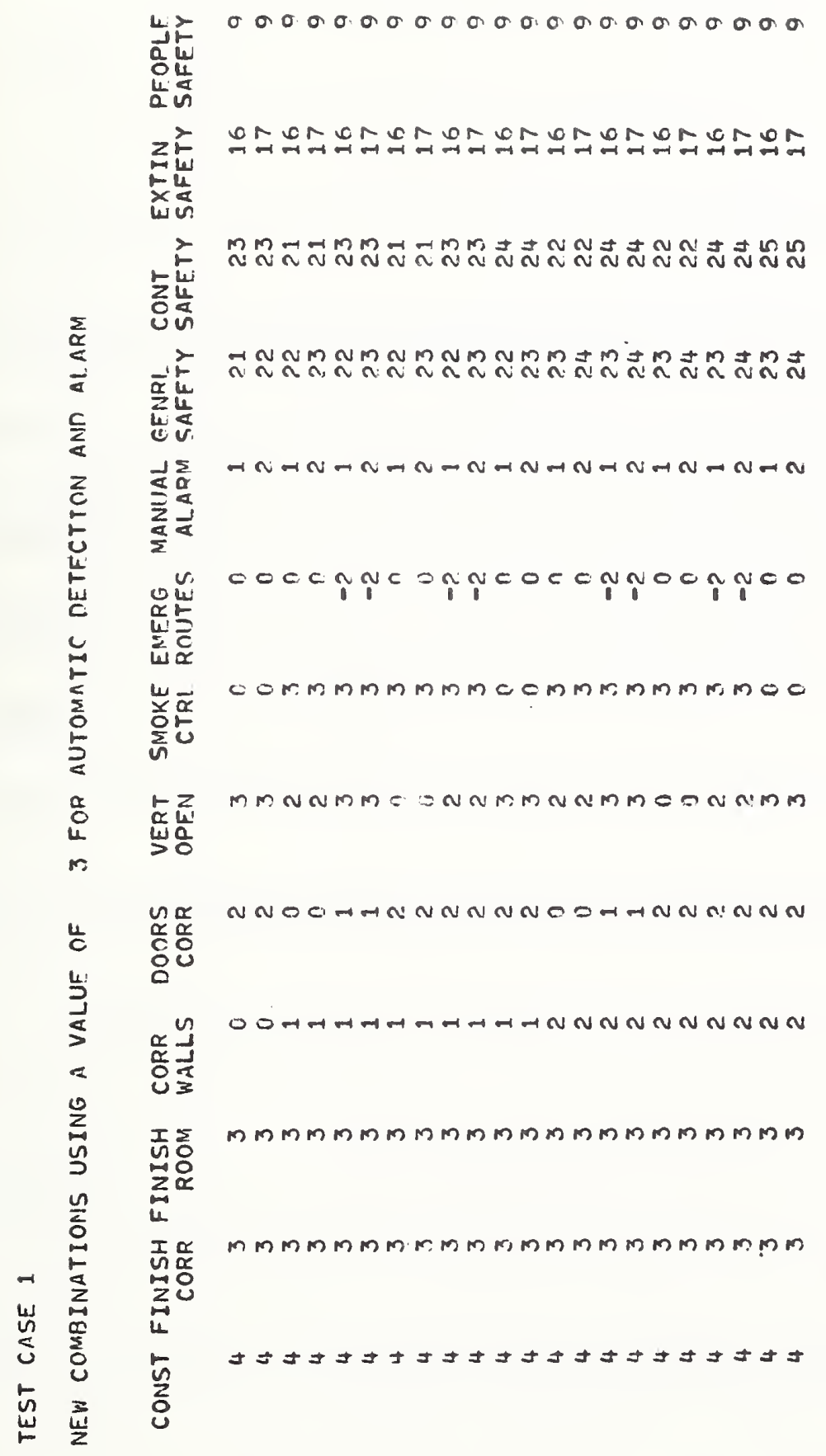


कब

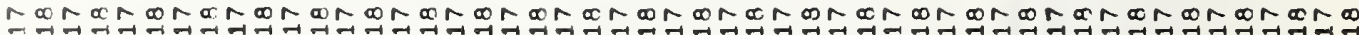

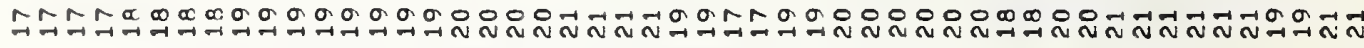

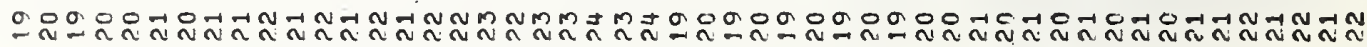

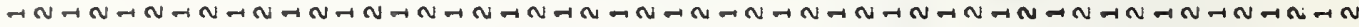

ऐ)

U.

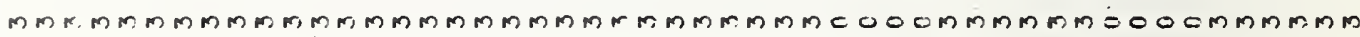

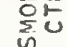

皆充

MMNMMMNNMMNNMMNNMMNNMMNNMMELNNMMNNMMEONNMMNNMMECNN

농

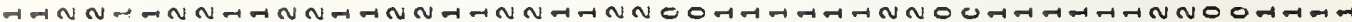
0

$\stackrel{\sim}{\frac{\pi}{0}}$

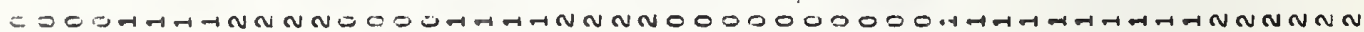
兵送 $\sum_{4}$

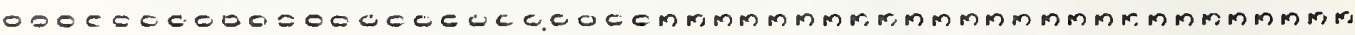
$\sum_{i=1}^{2}$ 


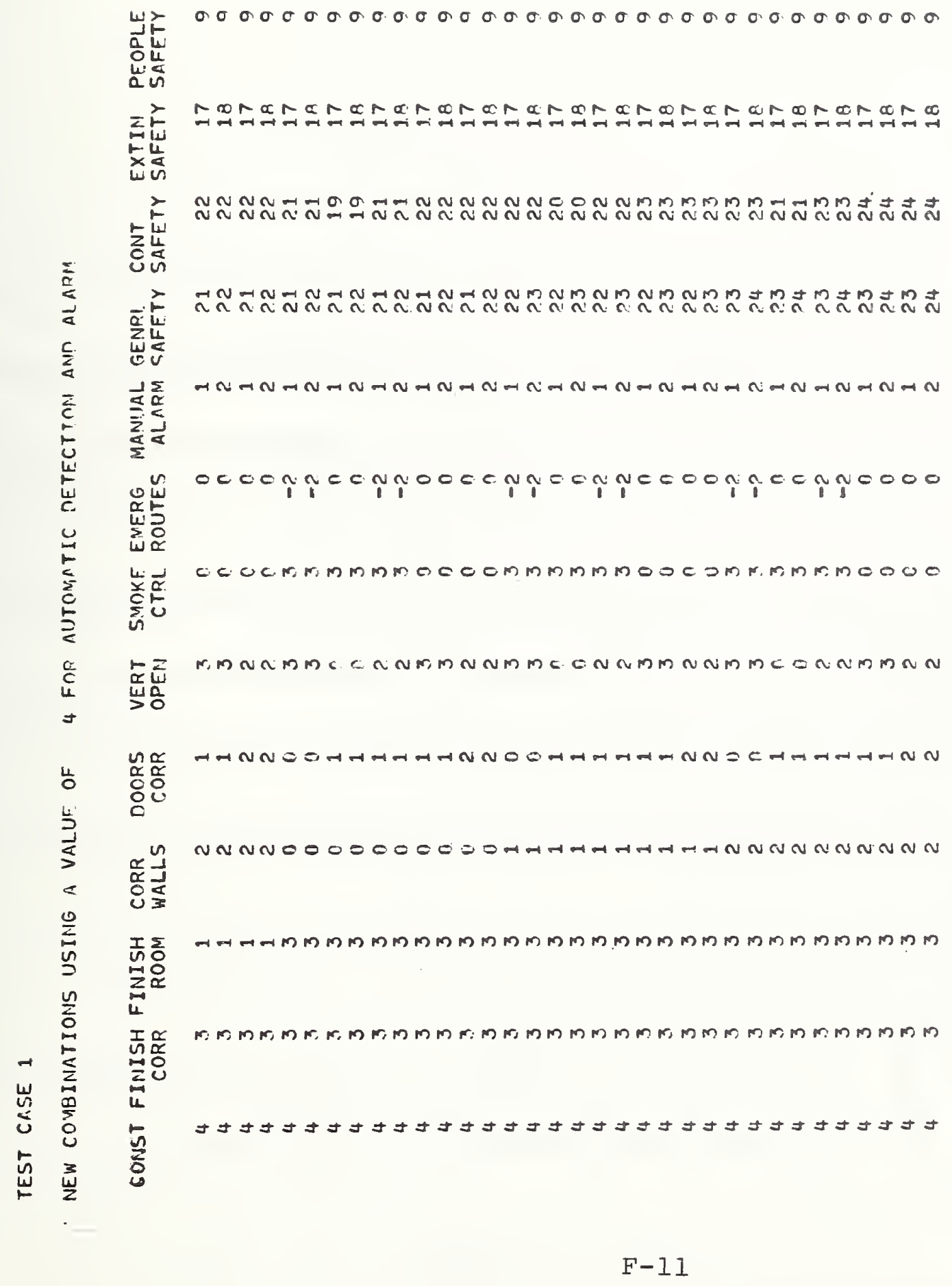


$\approx \approx \pm 00000000000000000000000000000000000000000000000$ is

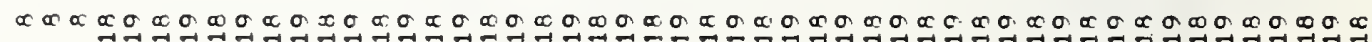

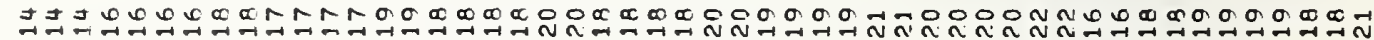
$\sum_{0} \frac{1}{2}$

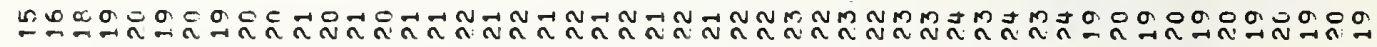

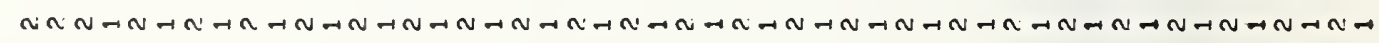
$\sum_{\sum} \frac{1}{d}$

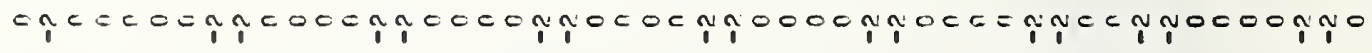
wळ 岩古

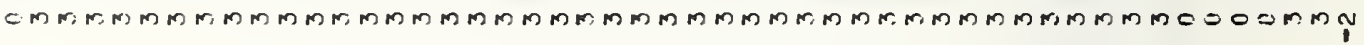
在空

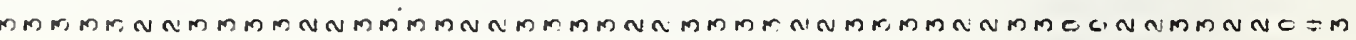

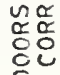

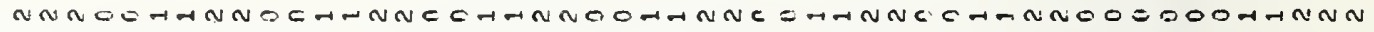

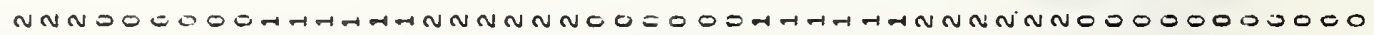

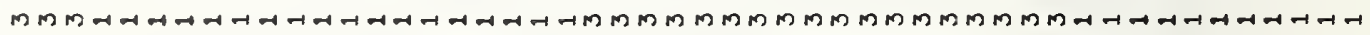
u.

In Mrriot $\stackrel{i=}{i}$ 
ब.

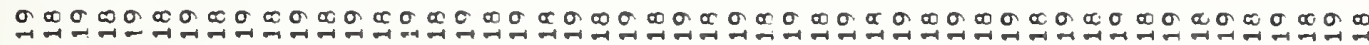

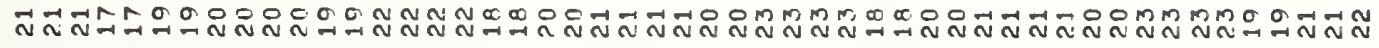

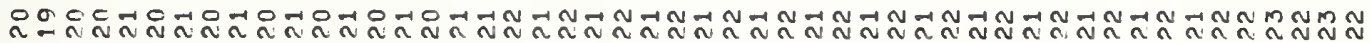

NGN

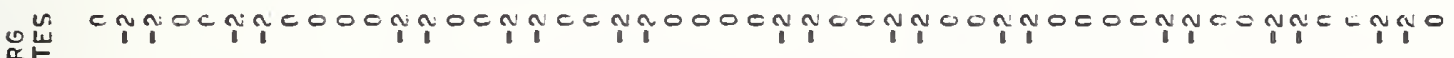

ᄂ̌ 赛品

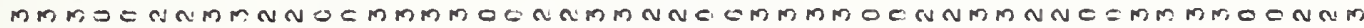

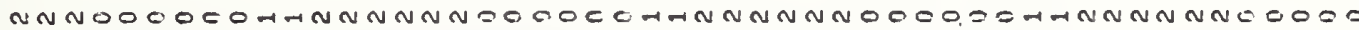

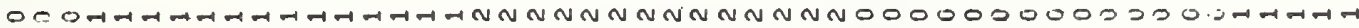

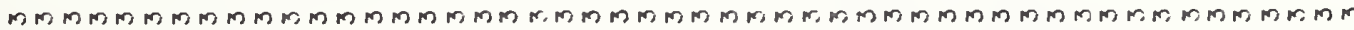

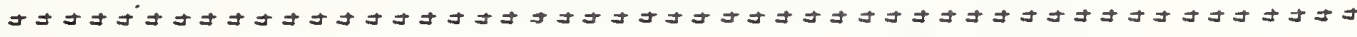


w

o.

$\sigma \approx \sigma a \sigma \propto \sigma \infty \sigma a \sigma \propto \sigma \propto \sigma \infty \sigma \infty \sigma \infty \sigma \infty \sigma$

$\sum_{m}$

$5<$

is

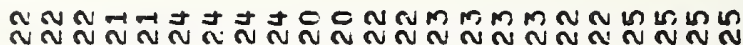

足

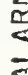

aे

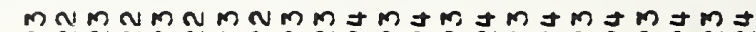

$\frac{2}{a}$

NAN

出

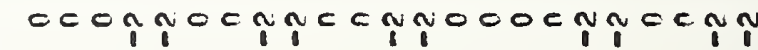

W $\sum_{n}^{c} u$

$5 z_{w}$

L

MNN

L

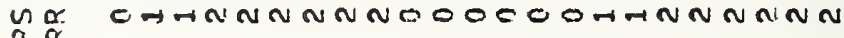

용용

Li

a न न

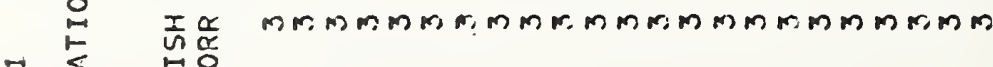

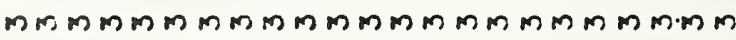

4

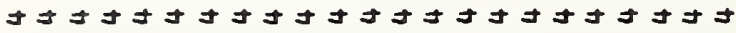

w $\frac{3}{2}$

$\frac{5}{5}$ 


\section{FEDERAL INFORMATION PROCESSING STANDARD SOFTWARE SUMMARY}

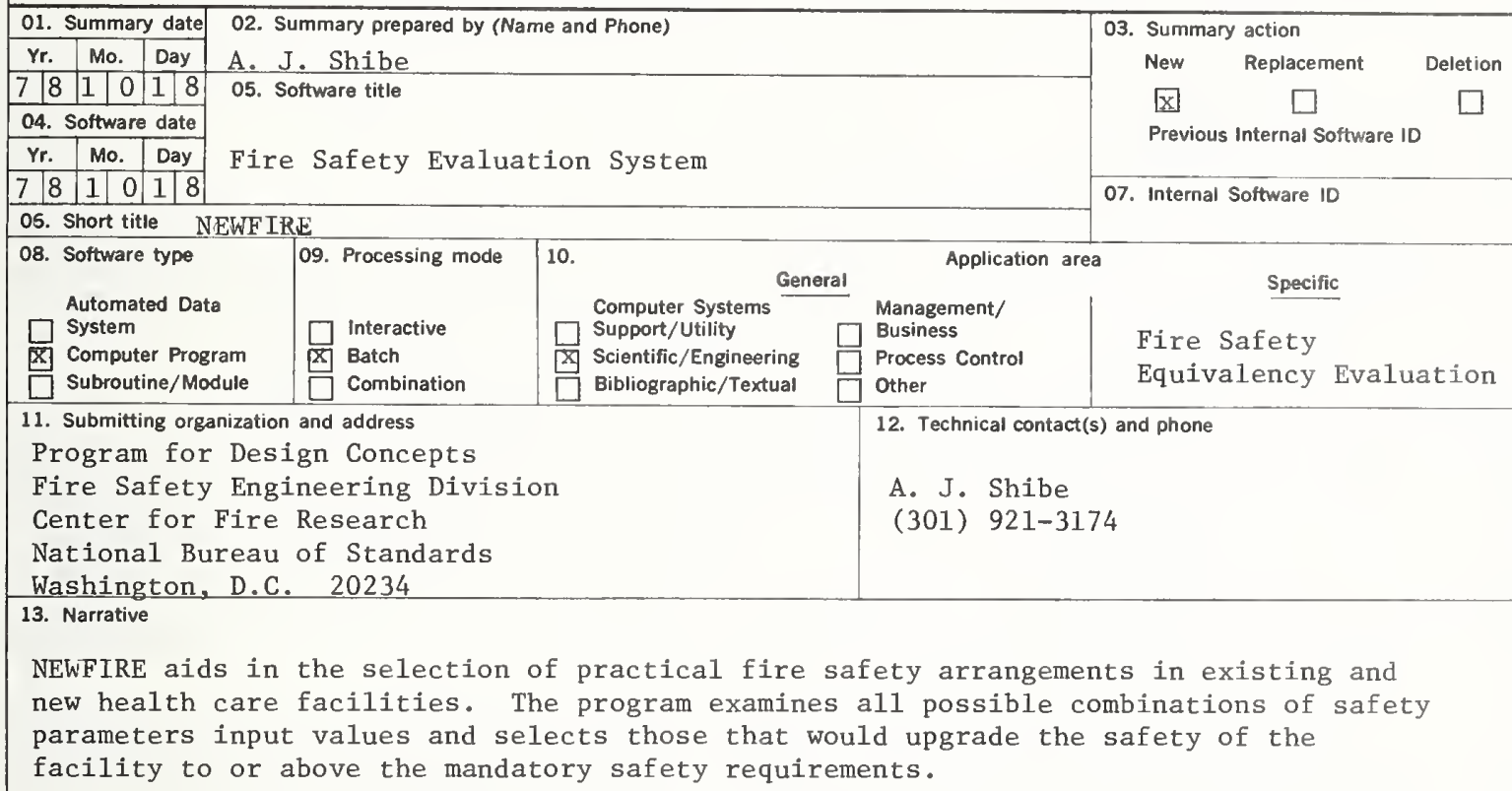

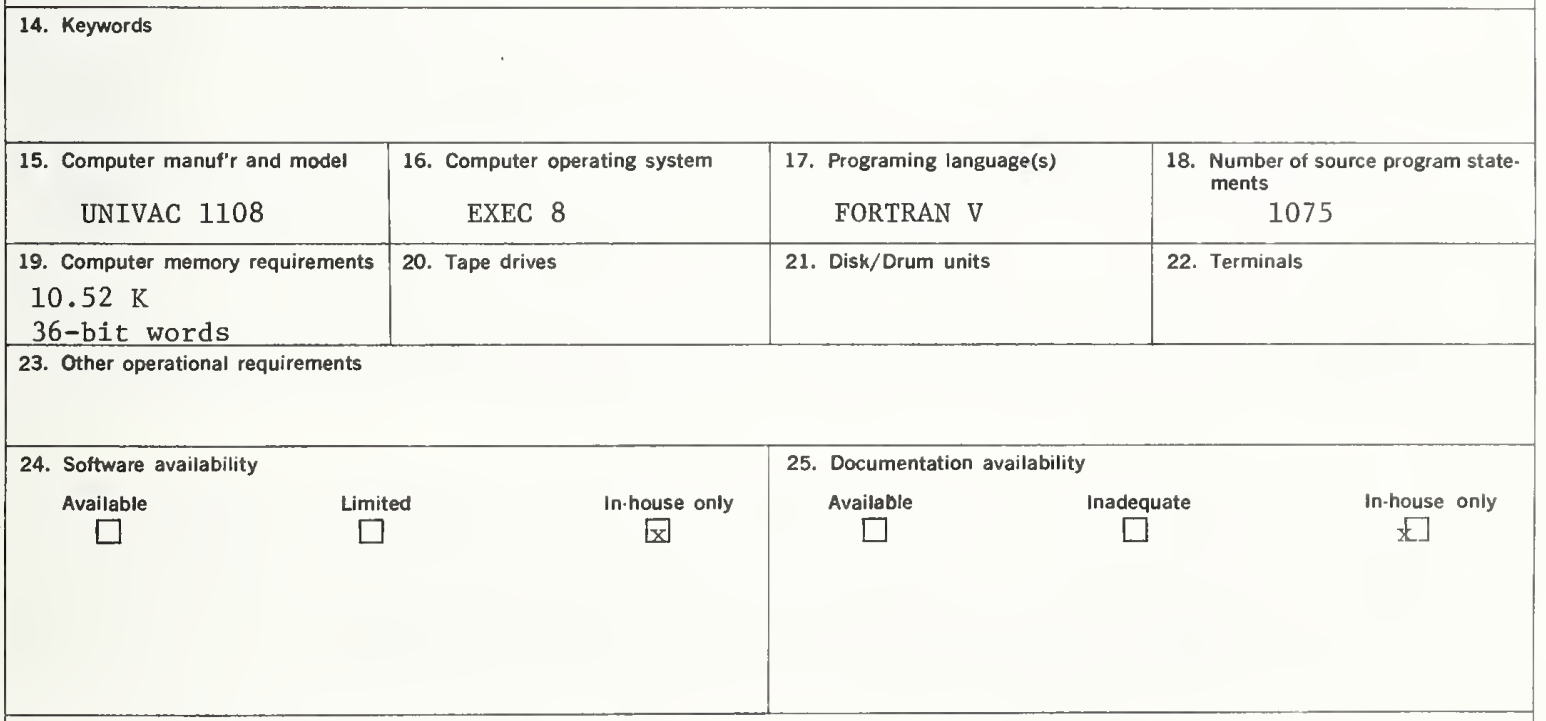

26. FOR SUBMITTING ORGANIZATION USE 

NBS-\{14A (REV.7-73)

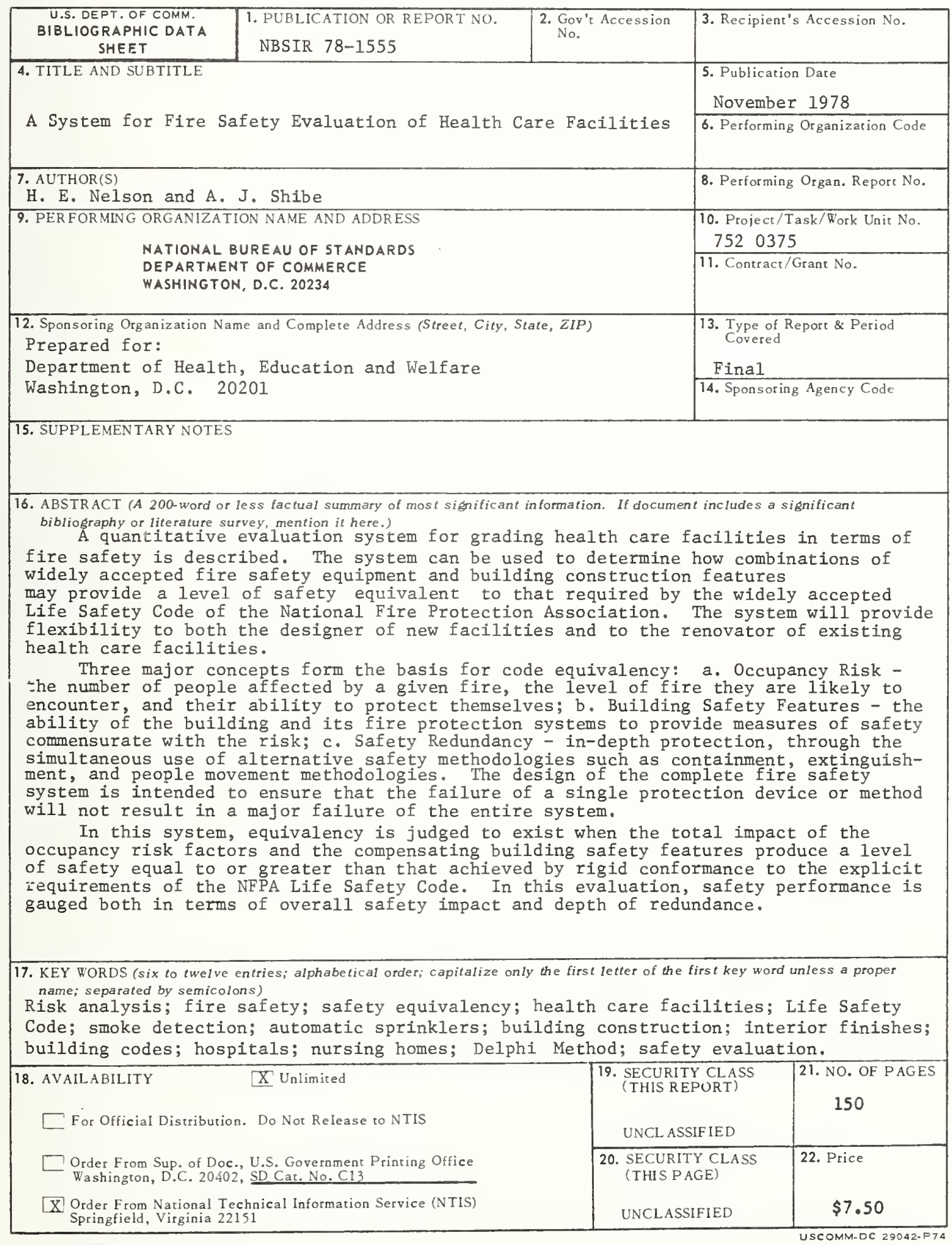




